\title{
A mixed finite element method for Darcy's equations with pressure dependent porosity*
}

\author{
Gabriel N. Gatica ${ }^{\dagger}$ Ricardo Ruiz-Baier ${ }^{\ddagger}$ Giordano Tierra ${ }^{\S}$
}

\begin{abstract}
In this work we develop the a priori and a posteriori error analyses of a mixed finite element method for Darcy's equations with porosity depending exponentially on the pressure. A simple change of variable for this unknown allows to transform the original nonlinear problem into a linear one whose dual-mixed variational formulation falls into the frameworks of the generalized linear saddle point problems and the fixed point equations satisfied by an affine mapping. According to the latter, we are able to show the well-posedness of both the continuous and discrete schemes, as well as the associated Cea estimate, by simply applying a suitable combination of the classical Babuška-Brezzi theory and the Banach fixed point Theorem. In particular, given any integer $k \geq 0$, the stability of the Galerkin scheme is guaranteed by employing Raviart-Thomas elements of order $k$ for the velocity, piecewise polynomials of degree $k$ for the pressure, and continuous piecewise polynomials of degree $k+1$ for an additional Lagrange multiplier given by the trace of the pressure on the Neumann boundary. Note that the two ways of writing the continuous formulation suggest accordingly two different methods for solving the discrete schemes. Next, we derive a reliable and efficient residualbased a posteriori error estimator for this problem. The global inf-sup condition satisfied by the continuous formulation, Helmholtz decompositions, and the local approximation properties of the Raviart-Thomas and Clément interpolation operators are the main tools for proving the reliability. In turn, inverse and discrete inequalities, and the localization technique based on triangle-bubble and edge-bubble functions are utilized to show the efficiency. Finally, several numerical results illustrating the good performance of both methods, confirming the aforementioned properties of the estimator, and showing the behaviour of the associated adaptive algorithm, are reported.
\end{abstract}

Key words: Darcy's equations, nonlinear problem, mixed-FEM, a posteriori error analysis Mathematics subject classifications (1991): 65N15, 65N30, 74F10, 74S05.

\section{Introduction}

The understanding and accurate rendering of fluid velocities and pressure of the porous medium is of key importance in diverse applications covering for instance, groundwater pollution, mezoscale blood

${ }^{*}$ This work was partially supported by BASAL project CMM, Universidad de Chile, by Centro de Investigación en Ingeniería Matemática ( $\mathrm{CI}^{2} \mathrm{MA}$ ), Universidad de Concepción, by CONICYT project Anillo ACT1118 (ANANUM), by the University of Lausanne, and by the Ministry of Education, Youth and Sports of the Czech Republic through the ERC-CZ project LL1202. Part of this research was developed while the third author was visiting $\mathrm{CI}^{2} \mathrm{MA}$ during the last three weeks of January 2014.

${ }^{\dagger} \mathrm{CI}^{2} \mathrm{MA}$ and Departamento de Ingeniería Matemática, Universidad de Concepción, Casilla 160-C, Concepción, Chile, email: ggatica@ci2ma.udec.cl

${ }^{\ddagger}$ Instite of Earth Sciences, Quartier UNIL-Mouline, Bâtiment Géopolis, University of Lausanne, CH-1015 Lausanne, Switzerland, e-mail: ricardo.ruizbaier@unil.ch

${ }^{\S}$ Mathematical Institute, Faculty of Mathematics and Physics, Charles University, Prague 8, 186 75, Czech Republic, e-mail: gtierra@karlin.mff.cuni.cz 
flows, filters design, enhanced oil recovery, carbon dioxide sequestration, and many others. In case of negligible relaxation time, and under relatively small permeability, it is possible to model the fluid flow in rigid porous media employing the well-known Darcy's law [22] which relates pressure gradient of the fully saturated porous medium with the velocity of the viscous incompressible fluid (volumetric flux). This model can be regarded as a starting point in the study of more involved and complex flow phenomena, and a myriad of numerical methods in different flavors and with increasingly appealing features has emerged in the past few decades. In particular, the development of suitable numerical methods for solving the Stokes-Darcy and related coupled problems, including porous media with cracks, the incorporation of the Brinkman equation in the model, and linear as well as nonlinear behaviors, has become a very active research area during the last decade (see, e.g. [13], [23], [25], [26], [31], [32], [37], [48], [55] and the references therein).

Our objective in this paper is to propose, analyze and implement a family of mixed finite element methods targeted to simulate the more general case where the porosity of the medium may depend explicitly (and exponentially) on the pressure, turning the classical Darcy's equations into a nonlinear system of equations. Indeed, the fact that the viscosity of a fluid could depend on pressure is very well established. For instance, as early as 1893, Barus was aware of these dependences [11], suggesting an exponential dependence of the viscosity on the pressure, i.e.,

$$
\mu(p)=\mu_{0} e^{\beta p}, \quad \mu_{0}>0, \beta \geq 0,
$$

where $\mu_{0}$ and $\beta$ are constants, with $\mu_{0}$ having the dimension of viscosity and $\beta$ the dimension inverse to that of the pressure. This pressure-dependent viscosity would lead to a drag coefficient $\alpha(p)$ of the form

$$
\alpha(p)=\alpha_{0} e^{\beta p}, \quad \alpha_{0} \geq 0 .
$$

We refer to [50] for a generalization of the classical Brinkman equation considering the dependence on pressure of the viscosity and the drag coefficient.

More precisely, in this work we are interested in the case in which the dissipation due to the drag at the pores is much larger than the dissipation due to the shear in the bulk fluid. Such a specific problem has been introduced in [47] and some attempts to approximate the system have been proposed lately. In fact, in [6] the authors present optimal error estimates for a spectral discretization of this system which takes into account the axisymmetry of the domain and of the flow. On the other hand, in [33] the authors consider first the simplified model in which the exponential law defining the porosity is truncated above and below by positive constants, whose corresponding solvability and regularity analysis was previously developed in [6], and propose a primal finite element scheme with polynomial approximations of degrees $k-1$ and $k$ for velocity and pressure, respectively. Then, the case of a fully exponential porosity is analyzed in the second part of [33], where, under the heuristic assumption that the resulting model has at least one solution, a suitable change of variables involving only the pressure allows to split the problem into two consecutive linear systems, which are discretized by slight variants of the method from the first part. Numerical results show the good performance of this approach. However, further regularity on the right-hand side datum needs to be assumed in order to derive a Robin-type boundary condition yielding the well-posedness of the continuous formulation. More recently, a strategy based on a posteriori error analysis and leading to an automatic identification of the subdomain where the pressure presents high variations, has been proposed in [2]. In this way, the iterative method needed to handle the nonlinear term is only applied in that region whereas the permeability is approximated by a constant in the remaining part. The resulting simplified model is then discretized in [2] by spectral elements as in [6], [7], and [21]. Other related works include [41], [42], and [18], where least squares and variational multiscale discretizations of Darcy's equations with pressure dependent viscosity have been extensively studied, and those referring to the nonlinear Darcy's system given by the well-known Forchheimer model (see, e.g. [35], [44], and [45]). 
Here we also consider the Darcy model with a fully exponential porosity from the second part of [33], assume again the heuristic hypothesis concerning the existence of solution, and apply the same change of variables employed there, but instead of a primal method, we opt for using the dual-mixed approach. As a consequence, the mixed boundary conditions arising from the transformation become readily employable and hence there is no need of additional regularity on the data nor of deriving any other boundary condition. In addition, the velocity unknowns of both problems coincide and the original pressure is recovered by a simple postprocessing formula depending only on the pressure of the transformed model. Moreover, the resulting dual-mixed variational formulation can be written as a generalized linear saddle point problem, and also as a fixed point equation satisfied by an affine mapping whose linear component is given by the solution of a usual linear saddle point problem. The first way of writing the continuous formulation emphasizes the fact that in this case the change of variables transforms the original nonlinear problem into a single linear one, whereas the second way allows us to show the well-posedness of both the continuous and discrete problems, as well as the associated Cea estimate, by simply combining the classical Babuška-Brezzi theory with the Banach fixed point Theorem. In addition, the above suggests two different numerical methods for the Galerkin scheme, namely a fixed point iterative procedure solving symmetric systems each time, and a direct solver of a single non-symmetric system.

Next, we derive a reliable and efficient residual-based a posteriori error estimator for the transformed problem and show the satisfactory performance of our method by means of several numerical tests. We remark that the application of adaptive algorithms based on a-posteriori error estimates aims to identify the regions with singularities or high gradients, so that further refinements are applied there at each stage, thus improving the convergence behavior of the Galerkin solutions of linear and nonlinear boundary value problems. The global estimator is normally represented by a quantity $\boldsymbol{\theta}$ depending on local estimators $\theta_{T}$ defined on each element $T$ of a given triangulation of the domain. Then, $\boldsymbol{\theta}$ is said to be reliable (resp. efficient) if there exists $C_{\text {rel }}>0$ (resp. $C_{\text {eff }}>0$ ), independent of the meshsizes, such that

$$
C_{\text {eff }} \boldsymbol{\theta}+\text { h.o.t. } \leq \| \text { error } \| \leq C_{\text {rel }} \boldsymbol{\theta}+\text { h.o.t. }
$$

where h.o.t. is a generic expression denoting one or several terms of higher order. While the list of references on a-posteriori error analysis for mixed formulations of linear and nonlinear problems is nowadays quite extensive, which includes several important contributions in recent years, most of the main ideas and associated techniques employed can be found in [3], [4], [14], [17], [30], [52], [54], and the references therein. In particular, the first corresponding results for elliptic partial differential equations of second order, which consider a-posteriori error estimators of explicit residual type, the solution of local problems, and the eventual derivation of reliability and efficiency properties, among other issues, go back to [52], [4], [14] and [17]. To this respect, we just mention here that the main tools for reliability and efficiency, which will also be employed below in Section 5, include Helmholtz decompositions, the localization technique based on bubble functions, discrete trace and inverse inequalities, and the approximation properties of the Clément interpolant.

The rest of this paper is organized as follows. We end this section with some notations to be used below. Section 2 introduces the nonlinear Darcy problem, which after a change of variables is written in mixed form. In Section 3 we derive the variational mixed formulation of the problem and establish its unique solvability using a fixed point argument. Section 4 is concerned with the construction and well-posedness analysis of a general Galerkin scheme for the discrete approximation of the modified problem, and we provide examples of finite element subspaces satisfying the underlying assumptions. A detailed residual-based a posteriori error analysis is carried out in Section 5, written specifically for the two-dimensional case. Finally, several examples are provided in Section 6, illustrating the accuracy of the aforementioned numerical methods, confirming the reliability and efficiency of the a 
posteriori error estimator previously derived, and showing the good performance of the associated adaptive algorithm.

In what follows we utilize standard simplified terminology for Sobolev spaces and norms. In particular, if $\mathcal{O} \subseteq \mathbb{R}^{d}(d \in\{2,3\})$ is a domain, $\mathcal{S} \subseteq \mathbb{R}^{d}$ is a Lipschitz curve or surface, and $r \in \mathbb{R}$, we define

$$
\boldsymbol{H}^{r}(\mathcal{O}):=\left[H^{r}(\mathcal{O})\right]^{d} \text { and } \quad \boldsymbol{H}^{r}(\mathcal{S}):=\left[H^{r}(\mathcal{S})\right]^{d} .
$$

However, when $r=0$ we usually write $\boldsymbol{L}^{2}(\mathcal{O})$ and $\boldsymbol{L}^{2}(\mathcal{S})$ instead of $\boldsymbol{H}^{0}(\mathcal{O})$ and $\boldsymbol{H}^{0}(\mathcal{S})$, respectively. The corresponding norms are denoted by $\|\cdot\|_{r, \mathcal{O}}\left(\right.$ for $H^{r}(\mathcal{O})$ and $\boldsymbol{H}^{r}(\mathcal{O})$ ) and $\|\cdot\|_{r, \mathcal{S}}\left(\right.$ for $H^{r}(\mathcal{S})$ and $\left.\boldsymbol{H}^{r}(\mathcal{S})\right)$. In general, given any Hilbert space $H$, we use $\boldsymbol{H}$ to denote $H^{d}$. In turn, the Hilbert space

$$
\boldsymbol{H}(\operatorname{div} ; \mathcal{O}):=\left\{\boldsymbol{w} \in \boldsymbol{L}^{2}(\mathcal{O}): \quad \operatorname{div} \boldsymbol{w} \in L^{2}(\mathcal{O})\right\}
$$

is standard in the realm of mixed problems (see [15]). Finally, we employ $\mathbf{0}$ to denote a generic null vector (including the null functional and operator), and use $C$ and $c$, with or without subscripts, bars, tildes or hats, to denote generic constants independent of the discretization parameters, which may take different values at different places.

\section{The model problem}

Let $\Omega \subseteq \mathbb{R}^{d}$ be a bounded domain $(d \in\{2,3\})$ with Lipschitz-boundary. The boundary of this domain is divided in two portions $\Gamma_{D}$ and $\Gamma_{N}$, such that $\Gamma_{D} \cap \Gamma_{N}=\emptyset$ and $\left|\Gamma_{D}\right|>0$, on which different types of boundary conditions will be imposed. We are interested in the model governing the flow of an incompressible fluid through a porous solid that can be derived within the context of mixtures, following e.g. [47]. The Cauchy stress $\mathbb{T}$ of the fluid is given by

$$
\mathbb{T}=-P \mathbb{I}+\mu \mathbb{D},
$$

where $P$ denotes the pressure of the fluid, $\mathbb{I}$ is the identity matrix of $\mathbb{R}^{d},-P \mathbb{I}$ is the indeterminate part of the stress due to the constraint of incompressibility, $\mu$ is the viscosity and $\mathbb{D}$ denotes the symmetric part of the velocity gradient, i.e.,

$$
\mathbb{D}=\frac{1}{2}\left(\nabla \boldsymbol{U}+(\nabla \boldsymbol{U})^{t}\right),
$$

where $\boldsymbol{U}$ is the velocity of the fluid. The balance of linear momentum for the fluid, is given by

$$
\rho \frac{\partial \boldsymbol{U}}{\partial t}-\operatorname{div} \mathbb{T}+\mathbb{F}=\rho \boldsymbol{f}
$$

where div is the usual divergence operator div acting row-wise, $\mathbb{F}$ is the interaction between the solid and the fluid, namely the frictional resistance at the pores of the solid on the fluid that is flowing. We shall assume that virtual mass effects, lift, and Basset forces can be neglected so that the only interaction mechanism is that due to the drag, that is

$$
\mathbb{F}=\alpha(P) \boldsymbol{U}
$$

where $\alpha$ is the pressure dependent permeability of the medium. Moreover, if the dissipation due to the drag at the pores is much larger than the dissipation due to the shear in the bulk fluid, it is reasonable to simplify the Cauchy stress to

$$
\mathbb{T}=-P \mathbb{I}
$$

We refer the reader to [51] for a thermodynamic basis for the derivation of models for flows through porous media and their generalizations. It follows from the previous relations, on neglecting the 
inertial term, that the appropriate governing equations supplemented by mixed boundary conditions are

$$
\begin{array}{ll}
\alpha(P) \boldsymbol{U}+\nabla P=\boldsymbol{f} & \text { in } \Omega, \\
\operatorname{div} \boldsymbol{U}=0 & \text { in } \Omega, \\
P=0 & \text { on } \Gamma_{D}, \\
\boldsymbol{U} \cdot \boldsymbol{\nu}=g & \text { on } \Gamma_{N},
\end{array}
$$

where $\boldsymbol{f} \in \boldsymbol{L}^{2}(\Omega)$ and $g \in H_{00}^{-1 / 2}\left(\Gamma_{N}\right)$ (see below for a definition of this space). Throughout this paper we also assume that $\alpha$ depends exponentially on $P$, that is, there exist constants $\alpha_{0}, \gamma>0$ such that

$$
\alpha(s)=\alpha_{0} e^{\gamma s} \quad \forall s \in \mathbb{R} .
$$

Hence, we rewrite the first equation of (2.1) as:

$$
\boldsymbol{U}=\frac{1}{\alpha(P)}(\boldsymbol{f}-\nabla P)=\frac{1}{\alpha_{0}}\left(e^{-\gamma P} \boldsymbol{f}-e^{-\gamma P} \nabla P\right)=\frac{1}{\alpha_{0}}\left(e^{-\gamma P} \boldsymbol{f}+\frac{1}{\gamma} \nabla\left(e^{-\gamma P}\right)\right),
$$

so that, assuming heuristically that (2.1) has at least one solution, and defining the new unknowns

$$
\boldsymbol{u}:=\boldsymbol{U} \quad \text { and } \quad p:=e^{-\gamma P}-1 \quad \text { in } \Omega,
$$

we can recast $(2.1)$ in the form

$$
\begin{array}{ll}
\boldsymbol{u}=\frac{1}{\alpha_{0}}(p+1) \boldsymbol{f}+\frac{1}{\alpha_{0} \gamma} \nabla p & \text { in } \Omega, \\
\operatorname{div} \boldsymbol{u}=0 & \text { in } \Omega, \\
p=0 & \text { on } \Gamma_{D}, \\
\boldsymbol{u} \cdot \boldsymbol{\nu}=g & \text { on } \Gamma_{N},
\end{array}
$$

Furthermore, we recall that the Sobolev space to which the Neumann datum $g$ belongs, that is $H_{00}^{-1 / 2}\left(\Gamma_{N}\right)$, is the dual of $H_{00}^{1 / 2}\left(\Gamma_{N}\right)$, where

$$
H_{00}^{1 / 2}\left(\Gamma_{N}\right):=\left\{\left.v\right|_{\Gamma_{N}}: \quad v \in H^{1}(\Omega), \quad v=0 \quad \text { on } \quad \Gamma_{D}\right\} .
$$

Alternatively, $H_{00}^{1 / 2}\left(\Gamma_{N}\right)$ is the interpolation space with index $1 / 2$ between $H_{0}^{1}\left(\Gamma_{N}\right)$ and $L^{2}\left(\Gamma_{N}\right)$ (cf. [38, Chapitre 1], [40, Appendix B]). In addition, from now on $\|\cdot\|_{0 ; 1 / 2, \Gamma_{N}}$ and $\|\cdot\|_{0 ;-1 / 2, \Gamma_{N}}$ denote the norms of $H_{00}^{1 / 2}\left(\Gamma_{N}\right)$ and $H_{00}^{-1 / 2}\left(\Gamma_{N}\right)$, respectively, and $\langle\cdot, \cdot\rangle_{\Gamma_{N}}$ stands for the corresponding duality pairing with respect to the $L^{2}\left(\Gamma_{N}\right)$ inner product.

\section{The variational formulation}

Multiplying by $\boldsymbol{v} \in \boldsymbol{H}(\operatorname{div} ; \Omega)$ the first equation of (2.2), integrating by parts, and introducing the additional unknown $\lambda:=-\left.p\right|_{\Gamma_{N}} \in H_{00}^{1 / 2}\left(\Gamma_{N}\right)$, we get

$$
\alpha_{0} \gamma \int_{\Omega} \boldsymbol{u} \cdot \boldsymbol{v}+\int_{\Omega} p \operatorname{div} \boldsymbol{v}=-\langle\boldsymbol{v} \cdot \boldsymbol{\nu}, \lambda\rangle_{\Gamma_{N}}+\gamma \int_{\Omega} p \boldsymbol{f} \cdot \boldsymbol{v}+\gamma \int_{\Omega} \boldsymbol{f} \cdot \boldsymbol{v} \quad \forall \boldsymbol{v} \in \boldsymbol{H}(\operatorname{div} ; \Omega) .
$$


In turn, from the second equation of $(2.2)$ we have

$$
\int_{\Omega} q \operatorname{div} \boldsymbol{u}=0 \quad \forall q \in L^{2}(\Omega),
$$

and imposing the Neumann condition in a weak fashion, we get

$$
\langle\boldsymbol{u} \cdot \boldsymbol{\nu}, \xi\rangle_{\Gamma_{N}}=\langle g, \xi\rangle_{\Gamma_{N}} \quad \forall \xi \in H_{00}^{1 / 2}\left(\Gamma_{N}\right) .
$$

In this way, we obtain the following saddle point problem: Find $(\boldsymbol{u},(p, \lambda)) \in \boldsymbol{H} \times \boldsymbol{Q}$ such that

$$
\begin{aligned}
\boldsymbol{a}(\boldsymbol{u}, \boldsymbol{v})+\boldsymbol{b}(\boldsymbol{v},(p, \lambda)) & =\gamma \int_{\Omega} p \boldsymbol{f} \cdot \boldsymbol{v}+\gamma \int_{\Omega} \boldsymbol{f} \cdot \boldsymbol{v} & & \forall \boldsymbol{v} \in \boldsymbol{H}, \\
\boldsymbol{b}(\boldsymbol{u},(q, \xi)) & =\langle g, \xi\rangle_{\Gamma_{N}} & & \forall(q, \xi) \in \boldsymbol{Q},
\end{aligned}
$$

where $\boldsymbol{H}:=\boldsymbol{H}(\operatorname{div} ; \Omega), \boldsymbol{Q}:=L^{2}(\Omega) \times H_{00}^{1 / 2}\left(\Gamma_{N}\right)$, and $\boldsymbol{a}: \boldsymbol{H} \times \boldsymbol{H} \rightarrow \mathbb{R}$ and $\boldsymbol{b}: \boldsymbol{H} \times \boldsymbol{Q} \rightarrow \mathbb{R}$ are the bounded bilinear forms defined by

$$
\begin{array}{ll}
\boldsymbol{a}(\boldsymbol{u}, \boldsymbol{v}):=\alpha_{0} \gamma \int_{\Omega} \boldsymbol{u} \cdot \boldsymbol{v} & \forall \boldsymbol{u}, \boldsymbol{v} \in \boldsymbol{H}, \\
\boldsymbol{b}(\boldsymbol{v},(q, \xi)):=\int_{\Omega} q \operatorname{div} \boldsymbol{v}+\langle\boldsymbol{v} \cdot \boldsymbol{\nu}, \xi\rangle_{\Gamma_{N}} & \forall(\boldsymbol{v},(q, \xi)) \in \boldsymbol{H} \times \boldsymbol{Q} .
\end{array}
$$

Note that actually (3.1) can be rewritten as: Find $(\boldsymbol{u},(p, \lambda)) \in \boldsymbol{H} \times \boldsymbol{Q}$ such that

$$
\begin{aligned}
\boldsymbol{a}(\boldsymbol{u}, \boldsymbol{v})+\boldsymbol{b}_{1}(\boldsymbol{v},(p, \lambda)) & =\gamma \int_{\Omega} \boldsymbol{f} \cdot \boldsymbol{v} & & \forall \boldsymbol{v} \in \boldsymbol{H}, \\
\boldsymbol{b}_{2}(\boldsymbol{u},(q, \xi)) & =\langle g, \xi\rangle_{\Gamma_{N}} & & \forall(q, \xi) \in \boldsymbol{Q},
\end{aligned}
$$

where $\boldsymbol{b}_{1}: \boldsymbol{H} \times \boldsymbol{Q} \rightarrow \mathbb{R}$ is the bounded bilinear form defined by

$$
\boldsymbol{b}_{1}(\boldsymbol{v},(q, \xi)):=\int_{\Omega} q \operatorname{div} \boldsymbol{v}+\langle\boldsymbol{v} \cdot \boldsymbol{\nu}, \xi\rangle_{\Gamma_{N}}-\gamma \int_{\Omega} q \boldsymbol{f} \cdot \boldsymbol{v} \quad \forall(\boldsymbol{v},(q, \xi)) \in \boldsymbol{H} \times \boldsymbol{Q},
$$

and $\boldsymbol{b}_{2}=\boldsymbol{b}$ (cf. (3.2)), which constitutes a particular example of the generalized Babuška-Brezzi theory developed in [12]. Nevertheless, for easiness of the analysis, in what follows we do not adopt this approach, but rather apply a combination of the classical Babuška-Brezzi theory and the Banach fixed point Theorem.

We now assume additionally that $\boldsymbol{f} \in \boldsymbol{L}^{\infty}(\Omega)$. Then, it is clear that, given $r \in L^{2}(\Omega)$, the linear functionals $\boldsymbol{F}_{r}: \boldsymbol{H} \rightarrow \mathbb{R}, \boldsymbol{F}: \boldsymbol{H} \rightarrow \mathbb{R}$ and $\boldsymbol{G}: \boldsymbol{Q} \rightarrow \mathbb{R}$ defined by

$$
\begin{gathered}
\boldsymbol{F}_{r}(\boldsymbol{v}):=\gamma \int_{\Omega} r \boldsymbol{f} \cdot \boldsymbol{v} \quad \forall \boldsymbol{v} \in \boldsymbol{H} \\
\boldsymbol{F}(\boldsymbol{v}):=\gamma \int_{\Omega} \boldsymbol{f} \cdot \boldsymbol{v} \quad \forall \boldsymbol{v} \in \boldsymbol{H}
\end{gathered}
$$

and

$$
\boldsymbol{G}(q, \xi):=\langle g, \xi\rangle_{\Gamma_{N}} \quad \forall(q, \xi) \in \boldsymbol{Q}
$$

satisfy

$$
\left|\boldsymbol{F}_{r}(\boldsymbol{v})\right| \leq \gamma\|r\|_{0, \Omega}\|\boldsymbol{f}\|_{\infty, \Omega}\|\boldsymbol{v}\|_{0, \Omega} \quad \forall \boldsymbol{v} \in \boldsymbol{H}
$$




$$
|\boldsymbol{F}(\boldsymbol{v})| \leq \gamma|\Omega|\|\boldsymbol{f}\|_{\infty, \Omega},\|\boldsymbol{v}\|_{0, \Omega} \quad \forall \boldsymbol{v} \in \boldsymbol{H}
$$

and

$$
|\boldsymbol{G}(q, \xi)| \leq\|g\|_{0 ;-1 / 2, \Gamma_{N}}\|\xi\|_{0 ; 1 / 2, \Gamma_{N}} \quad \forall(q, \xi) \in \boldsymbol{Q},
$$

which shows that $\boldsymbol{F}_{r} \in \boldsymbol{H}^{\prime}, \boldsymbol{F} \in \boldsymbol{H}^{\prime}$ and $\boldsymbol{G} \in \boldsymbol{Q}^{\prime}$. Also, there clearly holds

$$
|\boldsymbol{a}(\boldsymbol{u}, \boldsymbol{v})| \leq\|\boldsymbol{a}\|\|\boldsymbol{u}\|_{\boldsymbol{H}}\|\boldsymbol{v}\|_{\boldsymbol{H}} \quad \forall \boldsymbol{u}, \boldsymbol{v} \in \boldsymbol{H}
$$

and

$$
|\boldsymbol{b}(\boldsymbol{v},(q, \xi))| \leq\|\boldsymbol{b}\|\|\boldsymbol{v}\|_{\boldsymbol{H}}\|(q, \xi)\|_{\boldsymbol{Q}} \quad \forall(\boldsymbol{v},(q, \xi)) \in \boldsymbol{H} \times \boldsymbol{Q},
$$

with $\|\boldsymbol{a}\|=\alpha_{0} \gamma$ and $\|\boldsymbol{b}\|=1$. In addition, the kernel of $\boldsymbol{b}$ can be readily characterized as:

$$
\boldsymbol{V}:=\boldsymbol{N}(\boldsymbol{b})=\left\{\boldsymbol{v} \in \boldsymbol{H}(\operatorname{div} ; \Omega): \quad \operatorname{div} \boldsymbol{v}=0 \quad \text { in } \Omega, \quad \text { and } \boldsymbol{v} \cdot \boldsymbol{\nu}=0 \quad \text { on } \quad \Gamma_{N}\right\},
$$

and

$$
\boldsymbol{a}(\boldsymbol{v}, \boldsymbol{v})=\alpha_{0} \gamma\|\boldsymbol{v}\|_{0, \Omega}^{2}=\alpha\|\boldsymbol{v}\|_{\boldsymbol{H}}^{2} \quad \forall \boldsymbol{v} \in \boldsymbol{V},
$$

with $\alpha:=\alpha_{0} \gamma$, and it is quite standard to see that there exists $\beta>0$ such that (cf. [27, Section 2.4.2])

$$
\sup _{\substack{\boldsymbol{v} \in \boldsymbol{H} \\ \boldsymbol{v} \neq 0}} \frac{\boldsymbol{b}(\boldsymbol{v},(q, \xi))}{\|\boldsymbol{v}\|_{\boldsymbol{H}}} \geq \beta\|(q, \xi)\|_{\boldsymbol{Q}} \quad \forall(q, \xi) \in \boldsymbol{Q} .
$$

In other words, $\boldsymbol{a}(\cdot, \cdot)$ is $\boldsymbol{V}$-elliptic and $\boldsymbol{b}(\cdot, \cdot)$ satisfies the continuous inf-sup condition on $\boldsymbol{H} \times \boldsymbol{Q}$.

Consequently, we can introduce the affine operator $\boldsymbol{T}: \boldsymbol{H} \times \boldsymbol{Q} \rightarrow \boldsymbol{H} \times \boldsymbol{Q}$ that, given $(\boldsymbol{w},(r, \eta)) \in$ $\boldsymbol{H} \times \boldsymbol{Q}$, defines

$$
\boldsymbol{T}(\boldsymbol{w},(r, \eta)):=(\overline{\boldsymbol{u}},(\bar{p}, \bar{\lambda})) \in \boldsymbol{H} \times \boldsymbol{Q}
$$

as the unique solution of (3.1) when $p$ is replaced by $r$ on the right hand side of $(3.1)_{1}$, that is

$$
\begin{aligned}
\boldsymbol{a}(\overline{\boldsymbol{u}}, \boldsymbol{v})+\boldsymbol{b}(\boldsymbol{v},(\bar{p}, \bar{\lambda})) & =\boldsymbol{F}_{r}(\boldsymbol{v})+\boldsymbol{F}(\boldsymbol{v}) & & \forall \boldsymbol{v} \in \boldsymbol{H}, \\
\boldsymbol{b}(\overline{\boldsymbol{u}},(q, \xi)) & =\boldsymbol{G}(q, \xi) & & \forall(q, \xi) \in \boldsymbol{Q} .
\end{aligned}
$$

In this way, our original problem (3.1) can be rewritten as: Find $(\boldsymbol{u},(p, \lambda)) \in \boldsymbol{H} \times \boldsymbol{Q}$ such that

$$
\boldsymbol{T}(\boldsymbol{u},(p, \lambda))=(\boldsymbol{u},(p, \lambda))
$$

that is, to find a fixed point of $\boldsymbol{T}$, for which, as announced previously, we aim next to apply the Banach fixed point Theorem. To this end we first observe, from the superposition principle, that

$$
\boldsymbol{T}(\boldsymbol{w},(r, \eta))=\left(\boldsymbol{u}_{0},\left(p_{0}, \lambda_{0}\right)\right)+\boldsymbol{S}(\boldsymbol{w},(r, \eta)) \quad \forall(\boldsymbol{w},(r, \eta)) \in \boldsymbol{H} \times \boldsymbol{Q},
$$

where $\left(\boldsymbol{u}_{0},\left(p_{0}, \lambda_{0}\right)\right) \in \boldsymbol{H} \times \boldsymbol{Q}$ is the unique solution of the auxiliary problem

$$
\begin{aligned}
\boldsymbol{a}\left(\boldsymbol{u}_{0}, \boldsymbol{v}\right)+\boldsymbol{b}\left(\boldsymbol{v},\left(p_{0}, \lambda_{0}\right)\right) & =\boldsymbol{F}(\boldsymbol{v}) & & \forall \boldsymbol{v} \in \boldsymbol{H} \\
\boldsymbol{b}\left(\boldsymbol{u}_{0},(q, \xi)\right) & =\boldsymbol{G}(q, \xi) & & \forall(q, \xi) \in \boldsymbol{Q}
\end{aligned}
$$

and $\boldsymbol{S}: \boldsymbol{H} \times \boldsymbol{Q} \rightarrow \boldsymbol{H} \times \boldsymbol{Q}$ is the linear operator that, given $(\boldsymbol{w},(r, \eta)) \in \boldsymbol{H} \times \boldsymbol{Q}$, defines

$$
\boldsymbol{S}(\boldsymbol{w},(r, \eta)):=(\overline{\boldsymbol{u}},(\bar{p}, \bar{\lambda})) \in \boldsymbol{H} \times \boldsymbol{Q}
$$


as the unique solution of (3.10) with $\boldsymbol{F}$ and $\boldsymbol{G}$ replaced by null functionals, that is

$$
\begin{aligned}
\boldsymbol{a}(\overline{\boldsymbol{u}}, \boldsymbol{v})+\boldsymbol{b}(\boldsymbol{v},(\bar{p}, \bar{\lambda})) & =\boldsymbol{F}_{r}(\boldsymbol{v}) & & \forall \boldsymbol{v} \in \boldsymbol{H}, \\
\boldsymbol{b}(\overline{\boldsymbol{u}},(q, \xi)) & =0 & & \forall(q, \xi) \in \boldsymbol{Q} .
\end{aligned}
$$

Note here that (3.12) confirms the term affine given in advance to $\boldsymbol{T}$.

Furthermore, the continuous dependence result for (3.13) and (3.15) establishes the existence of a same constant $\tilde{C}:=\tilde{C}(\|\boldsymbol{a}\|, \alpha, \beta)>0$ such that

$$
\left\|\left(\boldsymbol{u}_{0},\left(p_{0}, \lambda_{0}\right)\right)\right\|_{\boldsymbol{H} \times \boldsymbol{Q}} \leq \tilde{C}\left\{\|\boldsymbol{F}\|_{\boldsymbol{H}^{\prime}}+\|\boldsymbol{G}\|_{\boldsymbol{Q}^{\prime}}\right\},
$$

and

$$
\|\boldsymbol{S}(\boldsymbol{w},(r, \eta))\|_{\boldsymbol{H} \times \boldsymbol{Q}} \leq \tilde{C}\left\|\boldsymbol{F}_{r}\right\|_{\boldsymbol{H}^{\prime}} .
$$

In particular, (3.17) and (3.6) yield

$$
\|\boldsymbol{S}(\boldsymbol{w},(r, \eta))\|_{\boldsymbol{H} \times \boldsymbol{Q}} \leq \tilde{C} \gamma\|\boldsymbol{f}\|_{\infty, \Omega}\|r\|_{0, \Omega} \quad \forall(\boldsymbol{w},(r, \eta)) \in \boldsymbol{H} \times \boldsymbol{Q},
$$

and hence, given $\left(\boldsymbol{w}_{1},\left(r_{1}, \eta_{1}\right)\right),\left(\boldsymbol{w}_{2},\left(r_{2}, \eta_{2}\right)\right) \in \boldsymbol{H} \times \boldsymbol{Q}$, we can use (3.12) to find that

$$
\begin{aligned}
\left\|\boldsymbol{T}\left(\boldsymbol{w}_{1},\left(r_{1}, \eta_{1}\right)\right)-\boldsymbol{T}\left(\boldsymbol{w}_{2},\left(r_{2}, \eta_{2}\right)\right)\right\|_{\boldsymbol{H} \times \boldsymbol{Q}} & =\left\|\boldsymbol{S}\left(\left(\boldsymbol{w}_{1},\left(r_{1}, \eta_{1}\right)\right)-\left(\boldsymbol{w}_{2},\left(r_{2}, \eta_{2}\right)\right)\right)\right\|_{\boldsymbol{H} \times \boldsymbol{Q}} \\
& \leq \tilde{C} \gamma\|\boldsymbol{f}\|_{\infty, \Omega}\left\|r_{1}-r_{2}\right\|_{0, \Omega} \\
& \leq \tilde{C} \gamma\|\boldsymbol{f}\|_{\infty, \Omega}\left\|\left(\boldsymbol{w}_{1},\left(r_{1}, \eta_{1}\right)\right)-\left(\boldsymbol{w}_{2},\left(r_{2}, \eta_{2}\right)\right)\right\|_{\boldsymbol{H} \times \boldsymbol{Q}},
\end{aligned}
$$

which shows that $\boldsymbol{T}$ is a contraction whenever

$$
\|\boldsymbol{f}\|_{\infty, \Omega}<\frac{1}{\tilde{C} \gamma} .
$$

We have thus proved the following result.

Theorem 3.1. Assume that $\boldsymbol{f} \in \boldsymbol{L}^{\infty}(\Omega)$ and that $\|\boldsymbol{f}\|_{\infty, \Omega}<\frac{1}{\tilde{C} \gamma}$. Then, there exists a unique $(\boldsymbol{u},(p, \lambda)) \in \boldsymbol{H} \times \boldsymbol{Q}$ solution of our variational formulation (3.1). Moreover, there holds

$$
\begin{aligned}
\|\boldsymbol{u}\|_{\boldsymbol{H}}+\left\{1-\tilde{C} \gamma\|\boldsymbol{f}\|_{\infty, \Omega}\right\}\|(p, \lambda)\|_{\boldsymbol{Q}} & \leq \tilde{C}\left\{\|\boldsymbol{F}\|_{\boldsymbol{H}^{\prime}}+\|\boldsymbol{G}\|_{\boldsymbol{Q}^{\prime}}\right\} \\
& \leq \tilde{C}\left\{\gamma|\Omega|\|\boldsymbol{f}\|_{\infty, \Omega}+\|g\|_{0 ;-1 / 2, \Gamma_{N}}\right\} .
\end{aligned}
$$

Proof. According to the foregoing analysis, the unique solvability of (3.1) follows straightforwardly from the Banach fixed point Theorem, and hence it only remains to show (3.18). For this purpose, we first observe, using (3.11) and (3.12), that

$$
\begin{aligned}
\|\boldsymbol{u}\|_{\boldsymbol{H}}+\|(p, \lambda)\|_{\boldsymbol{Q}} & =\|(\boldsymbol{u},(p, \lambda))\|_{\boldsymbol{H} \times \boldsymbol{Q}}=\|\boldsymbol{T}(\boldsymbol{u},(p, \lambda))\|_{\boldsymbol{H} \times \boldsymbol{Q}} \\
& =\left\|\left(\boldsymbol{u}_{0},\left(p_{0}, \lambda_{0}\right)\right)+\boldsymbol{S}(\boldsymbol{u},(p, \lambda))\right\|_{\boldsymbol{H} \times \boldsymbol{Q}},
\end{aligned}
$$

which, thanks to the triangle inequality and the estimates (3.16) and (3.17), leads to

$$
\|\boldsymbol{u}\|_{\boldsymbol{H}}+\|(p, \lambda)\|_{\boldsymbol{Q}} \leq \tilde{C}\left\{\|\boldsymbol{F}\|_{\boldsymbol{H}^{\prime}}+\|\boldsymbol{G}\|_{\boldsymbol{Q}^{\prime}}+\left\|\boldsymbol{F}_{p}\right\|_{\boldsymbol{H}^{\prime}}\right\} \text {. }
$$

In turn, it is clear from (3.6), (3.7), and (3.8), that

$$
\left\|\boldsymbol{F}_{p}\right\|_{\boldsymbol{H}^{\prime}} \leq \gamma\|\boldsymbol{f}\|_{\infty, \Omega}\|(p, \lambda)\|_{\boldsymbol{Q}}, \quad\|\boldsymbol{F}\|_{\boldsymbol{H}^{\prime}} \leq \gamma|\Omega|\|\boldsymbol{f}\|_{\infty, \Omega}, \quad \text { and } \quad\|\boldsymbol{G}\|_{\boldsymbol{Q}^{\prime}} \leq\|g\|_{0 ;-1 / 2, \Gamma_{N}}
$$

which, together with (3.19), yield the inequalities in (3.18) and complete the proof. 
In particular, if we assume in Theorem 3.1 that

$$
\|\boldsymbol{f}\|_{\infty, \Omega} \leq \frac{1}{2 \tilde{C} \gamma}
$$

we deduce that

$$
2\|\boldsymbol{u}\|_{\boldsymbol{H}}+\|(p, \lambda)\|_{\boldsymbol{Q}} \leq 2 \tilde{C}\left\{\|\boldsymbol{F}\|_{\boldsymbol{H}^{\prime}}+\|\boldsymbol{G}\|_{\boldsymbol{Q}^{\prime}}\right\}
$$

The converse of the derivation of (3.1) is provided now. More precisely, the following theorem establishes that the unique solution of (3.1) solves the original boundary value problem (2.2). This result is employed later on in Section 5.3 to show the efficiency of the a posteriori error estimator derived previously in Section 5.2.

Theorem 3.2. Let $(\boldsymbol{u},(p, \lambda)) \in \boldsymbol{H} \times \boldsymbol{Q}$ be the unique solution of (3.1). Then $\operatorname{div} \boldsymbol{u}=0$ in $\Omega, \boldsymbol{u} \cdot \boldsymbol{\nu}=g$ on $\Gamma_{N}, \nabla p=-\left(\gamma \boldsymbol{f}+\gamma p \boldsymbol{f}-\alpha_{0} \gamma \boldsymbol{u}\right)$ in $\Omega\left(\right.$ which yields $\left.p \in H^{1}(\Omega)\right), p=0$ on $\Gamma_{D}$, and $\lambda=-p$ on $\Gamma_{N}$.

Proof. It follows by applying integration by parts backwardly in (3.1) and then using suitable test functions. We omit further details.

\section{The Galerkin scheme}

\subsection{Main results}

Let $\boldsymbol{H}_{h}$ and $\boldsymbol{Q}_{h}$ be finite dimensional subspaces of $\boldsymbol{H}$ and $\boldsymbol{Q}$, respectively, and consider the Galerkin scheme: Find $\left(\boldsymbol{u}_{h},\left(p_{h}, \lambda_{h}\right)\right) \in \boldsymbol{H}_{h} \times \boldsymbol{Q}_{h}$ such that

$$
\begin{aligned}
\boldsymbol{a}\left(\boldsymbol{u}_{h}, \boldsymbol{v}_{h}\right)+\boldsymbol{b}\left(\boldsymbol{v}_{h},\left(p_{h}, \lambda_{h}\right)\right) & =\gamma \int_{\Omega} p_{h} \boldsymbol{f} \cdot \boldsymbol{v}_{h}+\gamma \int_{\Omega} \boldsymbol{f} \cdot \boldsymbol{v}_{h} & \forall \boldsymbol{v}_{h} \in \boldsymbol{H}_{h}, \\
\boldsymbol{b}\left(\boldsymbol{u}_{h},\left(q_{h}, \xi_{h}\right)\right) & =\left\langle g, \xi_{h}\right\rangle_{\Gamma_{N}} & \forall\left(q_{h}, \xi_{h}\right) \in \boldsymbol{Q}_{h} .
\end{aligned}
$$

Then, we let $\boldsymbol{V}_{h}$ be the discrete kernel of $\boldsymbol{b}$, that is

$$
\boldsymbol{V}_{h}:=\left\{\boldsymbol{v}_{h} \in \boldsymbol{H}_{h}: \quad \boldsymbol{b}\left(\boldsymbol{v}_{h},\left(q_{h}, \xi_{h}\right)\right)=0 \quad \forall\left(q_{h}, \xi_{h}\right) \in \boldsymbol{Q}_{h}\right\},
$$

and assume that there exists $\widehat{\alpha}>0$, independent of $h$, such that

$$
\boldsymbol{a}\left(\boldsymbol{v}_{h}, \boldsymbol{v}_{h}\right) \geq \widehat{\alpha}\left\|\boldsymbol{v}_{h}\right\|_{\boldsymbol{H}}^{2} \quad \forall \boldsymbol{v}_{h} \in \boldsymbol{V}_{h} .
$$

In addition, we also suppose that there exists $\widehat{\beta}>0$, independent of $h$, such that

$$
\sup _{\substack{\boldsymbol{v}_{h} \in \boldsymbol{H}_{h} \\ \boldsymbol{v}_{h} \neq 0}} \frac{\boldsymbol{b}\left(\boldsymbol{v}_{h},\left(q_{h}, \xi_{h}\right)\right)}{\left\|\boldsymbol{v}_{h}\right\|_{\boldsymbol{H}_{h}}} \geq \widehat{\beta}\left\|\left(q_{h}, \xi_{h}\right)\right\|_{\boldsymbol{Q}_{h}} \quad \forall\left(q_{h}, \xi_{h}\right) \in \boldsymbol{Q}_{h} .
$$

Specific finite element subspaces $\boldsymbol{H}_{h}$ and $\boldsymbol{Q}_{h}$ satisfying (4.3) and (4.4) will be indicated below in Section 4.2. Then, introducing corresponding discrete operators $\boldsymbol{T}_{h}: \boldsymbol{H}_{h} \times \boldsymbol{Q}_{h} \rightarrow \boldsymbol{H}_{h} \times \boldsymbol{Q}_{h}$ and $\boldsymbol{S}_{h}: \boldsymbol{H}_{h} \times \boldsymbol{Q}_{h} \rightarrow \boldsymbol{H}_{h} \times \boldsymbol{Q}_{h}$, and the particular discrete solutions $\left(\boldsymbol{u}_{h, 0},\left(p_{h, 0}, \lambda_{h, 0}\right)\right) \in \boldsymbol{H}_{h} \times \boldsymbol{Q}_{h}$, analogously to the definitions given for the continuous case (cf. (3.9), (3.12), (3.14)), we find that (4.1) is equivalent to the fixed point equation: Find $\left(\boldsymbol{u}_{h},\left(p_{h}, \lambda_{h}\right)\right) \in \boldsymbol{H}_{h} \times \boldsymbol{Q}_{h}$ such that

$$
\boldsymbol{T}_{h}\left(\boldsymbol{u}_{h},\left(p_{h}, \lambda_{h}\right)\right)=\left(\boldsymbol{u}_{h},\left(p_{h}, \lambda_{h}\right)\right)
$$


where

$$
\boldsymbol{T}_{h}\left(\boldsymbol{w}_{h},\left(r_{h}, \eta_{h}\right)\right)=\left(\boldsymbol{u}_{h, 0},\left(p_{h, 0}, \lambda_{h, 0}\right)\right)+\boldsymbol{S}_{h}\left(\boldsymbol{w}_{h},\left(r_{h}, \eta_{h}\right)\right) \quad \forall\left(\boldsymbol{w}_{h},\left(r_{h}, \eta_{h}\right)\right) \in \boldsymbol{H}_{h} \times \boldsymbol{Q}_{h} .
$$

Hence, employing basically the same arguments from the proof of Theorem 3.1, we deduce the following result.

Theorem 4.1. Assume that $\boldsymbol{f} \in \boldsymbol{L}^{\infty}(\Omega)$ and that $\|\boldsymbol{f}\|_{\infty, \Omega} \leq \frac{1}{2 \widehat{C} \gamma}$, where $\widehat{C}:=\widehat{C}(\|\boldsymbol{a}\|, \widehat{\alpha}, \widehat{\beta})$ is the continuous dependence constant for the discrete problems defining $\left(\boldsymbol{u}_{h, 0},\left(p_{h, 0}, \lambda_{h, 0}\right)\right)$ and the operator $\boldsymbol{S}_{h}$. Then, there exists a unique $\left(\boldsymbol{u}_{h},\left(p_{h}, \lambda_{h}\right)\right) \in \boldsymbol{H}_{h} \times \boldsymbol{Q}_{h}$ solution of (4.1), and there holds

$$
\begin{aligned}
2\left\|\boldsymbol{u}_{h}\right\|_{\boldsymbol{H}}+\left\|\left(p_{h}, \lambda_{h}\right)\right\|_{\boldsymbol{Q}} & \leq 2 \widehat{C}\left\{\left\|\left.\boldsymbol{F}\right|_{\boldsymbol{H}_{h}}\right\|_{\boldsymbol{H}_{h}^{\prime}}+\left\|\left.\boldsymbol{G}\right|_{\boldsymbol{Q}_{h}}\right\|_{\boldsymbol{Q}_{h}^{\prime}}\right\} \\
& \leq 2 \widehat{C}\left\{\gamma|\Omega|\|\boldsymbol{f}\|_{\infty, \Omega}+\|g\|_{0 ;-1 / 2, \Gamma_{N}}\right\} .
\end{aligned}
$$

On the other hand, in order to derive the Cea estimate for (4.1), we now take arbitrary functionals $\mathcal{F} \in \boldsymbol{H}^{\prime}, \mathcal{G} \in \boldsymbol{Q}^{\prime}, \mathcal{F}_{h} \in \boldsymbol{H}_{h}^{\prime}$, and $\mathcal{G}_{h} \in \boldsymbol{Q}_{h}^{\prime}$, and consider the following continuous and discrete problems:

(P) Find $(\boldsymbol{w},(r, \eta)) \in \boldsymbol{H} \times \boldsymbol{Q}$ such that

$$
\begin{aligned}
\boldsymbol{a}(\boldsymbol{w}, \boldsymbol{v})+\boldsymbol{b}(\boldsymbol{v},(r, \eta)) & =\mathcal{F}(\boldsymbol{v}) & & \forall \boldsymbol{v} \in \boldsymbol{H}, \\
\boldsymbol{b}(\boldsymbol{w},(q, \xi)) & =\mathcal{G}(q, \xi) & & \forall(q, \xi) \in \boldsymbol{Q} .
\end{aligned}
$$

$\left(\mathbf{P}_{h}\right)$ Find $\left(\boldsymbol{w}_{h},\left(r_{h}, \eta_{h}\right)\right) \in \boldsymbol{H}_{h} \times \boldsymbol{Q}_{h}$ such that

$$
\begin{aligned}
\boldsymbol{a}\left(\boldsymbol{w}_{h}, \boldsymbol{v}_{h}\right)+\boldsymbol{b}\left(\boldsymbol{v}_{h},\left(r_{h}, \eta_{h}\right)\right) & =\mathcal{F}_{h}\left(\boldsymbol{v}_{h}\right) & & \forall \boldsymbol{v}_{h} \in \boldsymbol{H}_{h}, \\
\boldsymbol{b}\left(\boldsymbol{w}_{h},\left(q_{h}, \xi_{h}\right)\right) & =\mathcal{G}_{h}\left(q_{h}, \xi_{h}\right) & & \forall\left(q_{h}, \xi_{h}\right) \in \boldsymbol{Q}_{h} .
\end{aligned}
$$

The corresponding Strang-type error estimate for $\mathbf{( P )}$ and $\left(\mathbf{P}_{h}\right)$ establishes in this case (cf. [49]) that

$$
\begin{aligned}
\left\|\boldsymbol{w}-\boldsymbol{w}_{h}\right\|_{\boldsymbol{H}} \leq & \left(1+\frac{\|\boldsymbol{a}\|}{\widehat{\alpha}}\right)\left(1+\frac{\|\boldsymbol{b}\|}{\widehat{\beta}}\right) \operatorname{dist}\left(\boldsymbol{w}, \boldsymbol{H}_{h}\right)+\frac{\|\boldsymbol{b}\|}{\widehat{\alpha}} \operatorname{dist}\left((r, \eta), \boldsymbol{Q}_{h}\right) \\
& +\frac{1}{\widehat{\beta}}\left(1+\frac{\|\boldsymbol{a}\|}{\widehat{\alpha}}\right)\left\|\mathcal{G}_{h}-\mathcal{G}\right\|_{\boldsymbol{Q}_{h}^{\prime}}+\frac{1}{\widehat{\alpha}}\left\|\mathcal{F}_{h}-\mathcal{F}\right\|_{\boldsymbol{H}_{h}^{\prime}}
\end{aligned}
$$

and

$$
\begin{aligned}
\left\|(r, \eta)-\left(r_{h}, \eta_{h}\right)\right\|_{\boldsymbol{Q}} \leq & \frac{\|\boldsymbol{a}\|}{\widehat{\beta}}\left(1+\frac{\|\boldsymbol{a}\|}{\widehat{\alpha}}\right)\left(1+\frac{\|\boldsymbol{b}\|}{\widehat{\beta}}\right) \operatorname{dist}\left(\boldsymbol{w}, \boldsymbol{H}_{h}\right) \\
& +\left(1+\frac{\|\boldsymbol{b}\|}{\widehat{\beta}}+\frac{\|\boldsymbol{a}\|\|\boldsymbol{b}\|}{\widehat{\alpha} \widehat{\beta}}\right) \operatorname{dist}\left((r, \eta), Q_{h}\right) \\
& +\frac{\|\boldsymbol{a}\|}{\widehat{\beta}^{2}}\left(1+\frac{\|\boldsymbol{a}\|}{\widehat{\alpha}}\right)\left\|\mathcal{G}_{h}-\mathcal{G}\right\|_{\boldsymbol{Q}_{h}^{\prime}}+\frac{1}{\widehat{\beta}}\left(1+\frac{\|\boldsymbol{a}\|}{\widehat{\alpha}}\right)\left\|\mathcal{F}_{h}-\mathcal{F}\right\|_{\boldsymbol{H}_{h}^{\prime}} .
\end{aligned}
$$

Hence, applying (4.5) and (4.6) to the continuous and discrete schemes given by (3.1) and (4.1), which means taking

$$
\begin{array}{rlrl}
\mathcal{F}(\boldsymbol{v}) & :=\gamma \int_{\Omega} p \boldsymbol{f} \cdot \boldsymbol{v}+\gamma \int_{\Omega} \boldsymbol{f} \cdot \boldsymbol{v}=\boldsymbol{F}_{p}(\boldsymbol{v})+\boldsymbol{F}(\boldsymbol{v}) & \forall \boldsymbol{v} \in \boldsymbol{H}, \\
\mathcal{F}_{h}(\boldsymbol{v}):=\gamma \int_{\Omega} p_{h} \boldsymbol{f} \cdot \boldsymbol{v}_{h}+\gamma \int_{\Omega} \boldsymbol{f} \cdot \boldsymbol{v}_{h}=\boldsymbol{F}_{p_{h}}\left(\boldsymbol{v}_{h}\right)+\boldsymbol{F}\left(\boldsymbol{v}_{h}\right) & \forall \boldsymbol{v}_{h} \in \boldsymbol{H}_{h},
\end{array}
$$


$\mathcal{G}:=\boldsymbol{G}$, and $G_{h}:=\left.\boldsymbol{G}\right|_{\boldsymbol{Q}_{h}}$, we deduce, with the constants $\widehat{C}_{1}, \widehat{C}_{2}, \widehat{C}_{3}$, and $\widehat{C}_{4}$ suggested by (4.5) and (4.6), that

$$
\left\|\boldsymbol{u}-\boldsymbol{u}_{h}\right\|_{\boldsymbol{H}} \leq \widehat{C}_{1} \operatorname{dist}\left(\boldsymbol{u}, \boldsymbol{H}_{h}\right)+\widehat{C}_{2} \operatorname{dist}\left((p, \lambda), \boldsymbol{Q}_{h}\right)+\frac{1}{\widehat{\alpha}}\left\|\boldsymbol{F}_{p-p_{h}}\right\|_{\boldsymbol{H}_{h}^{\prime}}
$$

and

$$
\left\|(p, \lambda)-\left(p_{h}, \lambda_{h}\right)\right\|_{\boldsymbol{Q}} \leq \widehat{C}_{3} \operatorname{dist}\left(\boldsymbol{u}, \boldsymbol{H}_{h}\right)+\widehat{C}_{4} \operatorname{dist}\left((p, \lambda), \boldsymbol{Q}_{h}\right)+\frac{1}{\widehat{\beta}}\left(1+\frac{\|\boldsymbol{a}\|}{\widehat{\alpha}}\right)\left\|\boldsymbol{F}_{p-p_{h}}\right\|_{\boldsymbol{H}_{h}^{\prime}},
$$

where, according to (3.6),

$$
\left\|\boldsymbol{F}_{p-p_{h}}\right\|_{\boldsymbol{H}_{h}^{\prime}} \leq \gamma\|\boldsymbol{f}\|_{\infty, \Omega}\left\|(p, \lambda)-\left(p_{h}, \lambda_{h}\right)\right\|_{\boldsymbol{Q}}
$$

Then, replacing (4.8) into (4.7), and assuming that

$$
\frac{\gamma}{\widehat{\beta}}\left(1+\frac{\|\boldsymbol{a}\|}{\widehat{\alpha}}\right)\|\boldsymbol{f}\|_{\infty, \Omega}=\frac{\gamma(\widehat{\alpha}+\|\boldsymbol{a}\|)}{\widehat{\alpha} \widehat{\beta}}\|\boldsymbol{f}\|_{\infty, \Omega}<1,
$$

we can write, in particular

$$
\|\boldsymbol{f}\|_{\infty, \Omega} \leq \frac{\widehat{\alpha} \widehat{\beta}}{2 \gamma(\widehat{\alpha}+\|\boldsymbol{a}\|)}
$$

and therefore we can assert that

$$
\left\|(p, \lambda)-\left(p_{h}, \lambda_{h}\right)\right\|_{\boldsymbol{Q}} \leq 2 \widehat{C}_{3} \operatorname{dist}\left(\boldsymbol{u}, \boldsymbol{H}_{h}\right)+2 \widehat{C}_{4} \operatorname{dist}\left((p, \lambda), \boldsymbol{Q}_{h}\right),
$$

and

$$
\left\|\boldsymbol{u}-\boldsymbol{u}_{h}\right\|_{\boldsymbol{H}} \leq \widehat{C}_{1} \operatorname{dist}\left(\boldsymbol{u}, \boldsymbol{H}_{h}\right)+\widehat{C}_{2} \operatorname{dist}\left((p, \lambda), \boldsymbol{Q}_{h}\right)+\frac{\gamma}{\widehat{\alpha}}\|\boldsymbol{f}\|_{\infty, \Omega}\left\|(p, \lambda)-\left(p_{h}, \lambda_{h}\right)\right\|_{\boldsymbol{Q}}
$$

Summarizing, and bearing in mind Theorems 3.1 and 4.1, we can state the following main result.

Theorem 4.2. Assume that $\boldsymbol{f} \in \boldsymbol{L}^{\infty}(\Omega)$ and that

$$
\|\boldsymbol{f}\|_{\infty, \Omega} \leq \frac{1}{2 \gamma} \min \left\{\frac{1}{\tilde{C}}, \frac{1}{\widehat{C}}, \frac{\widehat{\alpha} \widehat{\beta}}{\widehat{\alpha}+\|\boldsymbol{a}\|}\right\}
$$

where $\tilde{C}=\tilde{C}(\|\boldsymbol{a}\|, \alpha, \beta)>0$ and $\widehat{C}=\widehat{C}(\|\boldsymbol{a}\|, \widehat{\alpha}, \widehat{\beta})>0$ are the continuous dependence constants specified above. Then, the continuous and discrete problems (3.1) and (4.1) have unique solutions $(\boldsymbol{u},(p, \lambda)) \in \boldsymbol{H} \times \boldsymbol{Q}$ and $\left(\boldsymbol{u}_{h},\left(p_{h}, \lambda_{h}\right)\right) \in \boldsymbol{H}_{h} \times \boldsymbol{Q}_{h}$. Moreover, there exists a constant $C>0$, depending on $\|\boldsymbol{a}\|,\|\boldsymbol{b}\|, \widehat{\alpha}, \widehat{\beta}, \gamma$, and $\|\boldsymbol{f}\|_{\infty, \Omega}$, such that

$$
\left\|(\boldsymbol{u},(p, \lambda))-\left(\boldsymbol{u}_{h},\left(p_{h}, \lambda_{h}\right)\right)\right\|_{\boldsymbol{H} \times \boldsymbol{Q}} \leq C \operatorname{dist}\left((\boldsymbol{u},(p, \lambda)), \boldsymbol{H}_{h} \times \boldsymbol{Q}_{h}\right) .
$$




\subsection{Specific finite element subspaces}

We now assume that $\Omega$ is a polyhedral region of $\mathbb{R}^{d}$ and proceed to define explicit finite element subspaces of $\boldsymbol{H}:=\boldsymbol{H}(\operatorname{div} ; \Omega)$ and $\boldsymbol{Q}:=L^{2}(\Omega) \times H_{00}^{1 / 2}\left(\Gamma_{N}\right)$ satisfying the $\boldsymbol{V}_{h}$-ellipticity (4.3) and the discrete inf-sup condition (4.4). For this purpose, we let $\left\{\mathcal{T}_{h}\right\}_{h>0}$ be a shape-regular family of dscretizations of $\bar{\Omega}$ into triangles $T$ (in $\mathbb{R}^{2}$ ) or tetrahedra $T$ (in $\mathbb{R}^{3}$ ) of diameter $h_{T}$, with meshsize

$h:=\max \left\{h_{T}: \quad T \in \mathcal{T}_{h}\right\}$, and such that the partitions of $\Gamma_{N}$ and $\Gamma_{D}$ inherited from $\mathcal{T}_{h}$ coincide on $\bar{\Gamma}_{D} \cap \bar{\Gamma}_{N}$. Also, given an integer $k \geq 0$ and a subset $S$ of $\mathbb{R}^{d}$, we denote by $P_{k}(S)$ the space of polynomials defined in $S$ of total degree at most $k$. Then, for each integer $k \geq 0$ and for each $T \in \mathcal{T}_{h}$, we recall the definition of the local Raviart-Thomas space of order $k$ as (see, e.g. [15], [49])

$$
\mathbf{R T}_{k}(T):=\mathbf{P}_{k}(T) \oplus P_{k}(T) \boldsymbol{x}
$$

where $\boldsymbol{x}:=\left(\begin{array}{c}x_{1} \\ \vdots \\ x_{d}\end{array}\right)$ is a generic vector of $\mathbb{R}^{d}$,

$$
P_{k}(T) \boldsymbol{x}:=\left\{\mathbf{q}: T \rightarrow \mathbb{R}^{d}: \mathbf{q}(\boldsymbol{x}):=p(\boldsymbol{x}) \boldsymbol{x} \forall \boldsymbol{x} \in T, \text { for some } p \in P_{k}(T)\right\},
$$

and, according to the notation introduced in Section $1, \mathbf{P}_{k}(T):=\left[P_{k}(T)\right]^{d}$.

Then, given an integer $k \geq 0$, we define the finite element subspace $\boldsymbol{H}_{h}$ for the approximation of $\boldsymbol{u} \in \boldsymbol{H}(\operatorname{div} ; \Omega)$ as the global Raviart-Thomas space of order $k$, that is,

$$
\boldsymbol{H}_{h}:=\left\{\boldsymbol{v}_{h} \in \boldsymbol{H}(\operatorname{div} ; \Omega):\left.\quad \boldsymbol{v}_{h}\right|_{T} \in \mathbf{R T}_{k}(T) \quad \forall T \in \mathcal{T}_{h}\right\}
$$

In turn, the finite element subspace for the pressure $p$ is given by the global space of piecewise polynomials of degree $\leq k$, that is

$$
Q_{h}^{p}:=\left\{q_{h} \in L^{2}(\Omega):\left.\quad q_{h}\right|_{T} \in P_{k}(T) \quad \forall T \in \mathcal{T}_{h}\right\}
$$

Next, in order to define the finite element subspace for $\lambda$, we first let $\Gamma_{N, h}$ be the partition of $\Gamma_{N}$ inherited from the triangulation $\mathcal{T}_{h}$, and define the meshsize $h_{N}:=\max \left\{|e|: \quad e \in \Gamma_{N, h}\right\}$. Note here that $e$ denotes either edges of triangles $\left(\right.$ in $\mathbb{R}^{2}$ ) or faces of tetrahedra (in $\mathbb{R}^{3}$ ). Then, we let $\Gamma_{N, \tilde{h}}$ be another partition of $\Gamma_{N}$, independent of $\Gamma_{N, h}$, and define $\tilde{h}_{N}:=\max \left\{|e|: \quad e \in \Gamma_{N, \tilde{h}}\right\}$. Then, for the same integer $k \geq 0$ employed in the definitions (4.9) and (4.10), we introduce

$$
Q_{\tilde{h}}^{\lambda}:=\left\{\xi_{\tilde{h}} \in H_{00}^{1 / 2}\left(\Gamma_{N}\right):\left.\quad \xi_{\tilde{h}}\right|_{e} \in P_{k+1}(e) \quad \forall e \in \Gamma_{N, \tilde{h}}\right\}
$$

and then set

$$
\boldsymbol{Q}_{h, \tilde{h}}:=Q_{h}^{p} \times Q_{\tilde{h}}^{\lambda}
$$

According to the above definitions of $\boldsymbol{H}_{h}$ and $Q_{h}^{p}$, it is clear that $\operatorname{div} \boldsymbol{H}_{h} \subseteq Q_{h}^{p}$, which implies that the discrete kernel of $\boldsymbol{b}$ (cf. (4.2)) becomes

$$
\boldsymbol{V}_{h}:=\left\{\boldsymbol{v}_{h} \in \boldsymbol{H}_{h}: \quad \operatorname{div} \boldsymbol{v}_{h}=0 \quad \text { in } \quad \Omega, \quad \text { and } \quad\left\langle\boldsymbol{v}_{h} \cdot \boldsymbol{\nu}, \xi_{\tilde{h}}\right\rangle_{\Gamma_{N}}=0 \quad \forall \xi_{\tilde{h}} \in Q_{\tilde{h}}^{\lambda}\right\}
$$

and hence the $\boldsymbol{V}_{h}$-ellipticity of $\boldsymbol{a}$ (cf. (4.3)) follows straightforwardly from the free divergence property satisfied by this subspace. Though it is not required by any subsequent analysis, we notice that the second condition defining $\boldsymbol{V}_{h}$ does not necessarily imply that $\boldsymbol{v}_{h} \cdot \boldsymbol{\nu}$ vanishes on $\Gamma_{N}$ for each $\boldsymbol{v}_{h} \in \boldsymbol{V}_{h}$. 
Now, concerning the discrete inf-sup condition for $\boldsymbol{b}$ (cf. (4.4)), we omit detailed explanations and just observe that it actually follows from a slight modification of the analysis provided in [9, Lemma $3.1,3.2$, and 3.3] for the case $k=0$ in $\mathbb{R}^{2}$. More precisely, assuming additionally that $\left\{\mathcal{T}_{h}\right\}_{h>0}$ is quasiuniform in a neighborhood of $\Gamma_{N}$, the arguments from [9] can be easily extended to $\mathbb{R}^{d}(d \in\{2,3\})$ and for any integer $k \geq 1$, thus showing the existence of constants $\left.\left.C_{0} \in\right] 0,1\right]$ and $\widehat{\beta}>0$, both independent of $h$ and $\tilde{h}$, such that (4.4) holds whenever $h_{N} \leq C_{0} \tilde{h}_{N}$. Certainly, this restriction on the meshsizes should be incorporated in the statements of Theorems 4.1 and 4.2 when the subspaces $\boldsymbol{H}_{h}$ and $\boldsymbol{Q}_{h, \tilde{h}}$ given by (4.9) and (4.12) are employed.

In addition to the foregoing discussion, it is important to remark that a simplification in the definition of the finite element subspace for $\lambda$ is possible in the two-dimensional case when $k=0$. In fact, let us first assume, without loss of generality, that the number of edges of $\Gamma_{N, h}$ is an even number. The case of an odd number of edges is easily reduced to the even one by replacing any particular pair of adjacent edges by a single edge. Then, we let $\Gamma_{N, 2 h}$ be the partition of $\Gamma_{N}$ arising by joining pairs of adjacent elements of $\Gamma_{N, h}$, and define

$$
Q_{h}^{\lambda}:=\left\{\xi_{h} \in H_{00}^{1 / 2}\left(\Gamma_{N}\right):\left.\quad \xi_{h}\right|_{e} \in P_{1}(e) \quad \forall e \in \Gamma_{N, 2 h}\right\}
$$

so that instead of $\boldsymbol{Q}_{h, \tilde{h}}$ we now set

$$
\boldsymbol{Q}_{h}:=Q_{h}^{p} \times Q_{h}^{\lambda} .
$$

In this case, the discrete inf-sup condition for $\boldsymbol{b}$ (cf. (4.4)) does not need any quasi-uniformity assumption around $\Gamma_{N}$ nor any restriction on the meshsizes, and it basically follows by applying [32, Lemma 4.2], the analysis from [32, Section 5.1], and the recent result provided by [39, Theorem 5.1].

We end this description of specific finite element subspaces by mentioning that, for practical purposes, particularly for the implementation of the examples reported below in Section 6, the restriction on the meshsizes required by the discrete inf-sup condition (4.4) (for $k \geq 1$ in $\mathbb{R}^{2}$, and for $k \geq 0$ in $\mathbb{R}^{3}$ ) is verified in an heuristic sense only. More precisely, since the constant $C_{0}$ involved there is actually unknown, we simply assume $C_{0}=1 / 2$ and consider a partition $\Gamma_{N, \tilde{h}}$ with a meshsize $\tilde{h}_{N}$ given approximately by the double of $h_{N}$. The numerical results to be provided in that section will confirm the suitability of this choice.

\section{$5 \quad$ A posteriori error analysis}

In this section we derive a reliable and efficient residual based a posteriori error estimator for (4.1). For simplicity, we restrict our analysis to the two-dimensional case with $k=0$, which means that throughout this section our finite element subspaces $\boldsymbol{H}_{h}, Q_{h}^{p}$, and $Q_{h}^{\lambda}$ are given by (4.9) (with $k=0$ ), (4.10) (with $k=0$ ), and (4.13), respectively. Minor modifications allow to extend our approach to higher polynomial approximations and to $\mathbb{R}^{3}$. We begin by introducing several notations. We let $\mathcal{E}_{h}$ be the set of all edges of the triangulation $\mathcal{T}_{h}$, and given $T \in \mathcal{T}_{h}$, we let $\mathcal{E}(T)$ be the set of its edges. Then we write $\mathcal{E}_{h}=\mathcal{E}_{h}(\Omega) \cup \mathcal{E}_{h}\left(\Gamma_{D}\right) \cup \mathcal{E}_{h}\left(\Gamma_{N}\right)$, where $\mathcal{E}_{h}(\Omega):=\left\{e \in \mathcal{E}_{h}: \quad e \subseteq \Omega\right\}$, $\mathcal{E}_{h}\left(\Gamma_{D}\right):=\left\{e \in \mathcal{E}_{h}: e \subseteq \Gamma_{D}\right\}$, and analogously for $\mathcal{E}_{h}\left(\Gamma_{N}\right)$. In what follows, $h_{e}$ stands for the length of a given edge $e$. Also, for each edge $e \in \mathcal{E}_{h}$ we fix a unit normal vector $\nu_{e}:=\left(\nu_{1}, \nu_{2}\right)^{\mathrm{t}}$, and let $\boldsymbol{s}_{e}:=\left(-\nu_{2}, \nu_{1}\right)^{\mathrm{t}}$ be the corresponding fixed unit tangential vector along $e$. However, when no confusion arises, we simple write $\boldsymbol{\nu}$ and $\boldsymbol{s}$ instead of $\boldsymbol{\nu}_{e}$ and $\boldsymbol{s}_{e}$, respectively. Now, let $\boldsymbol{v} \in \boldsymbol{L}^{2}(\Omega)$ such that $\left.\boldsymbol{v}\right|_{T} \in \boldsymbol{C}(T)$ on each $T \in \mathcal{T}_{h}$. Then, given $T \in \mathcal{T}_{h}$ and $e \in \mathcal{E}(T) \cap \mathcal{E}_{h}(\Omega)$, we denote by $[\boldsymbol{v} \cdot \boldsymbol{s}]$ the tangential jump of $\boldsymbol{v}$ across $e$, that is $[\boldsymbol{v} \cdot \boldsymbol{s}]:=\left.\left(\left.\boldsymbol{v}\right|_{T}-\left.\boldsymbol{v}\right|_{T^{\prime}}\right)\right|_{e} \cdot \boldsymbol{s}$, where $T$ and $T^{\prime}$ are the triangles of $\mathcal{T}_{h}$ having $e$ as a common edge. Similar definitions hold for the tangential jumps of scalar fields 
$\varphi \in L^{2}(\Omega)$ such that $\left.\varphi\right|_{T} \in C(T)$ on each $T \in \mathcal{T}_{h}$. Finally, given scalar and vector valued fields $\varphi$ and $\boldsymbol{v}:=\left(v_{1}, v_{2}\right)^{\mathrm{t}}$, respectively we let

$$
\operatorname{curl} \varphi:=\left(\begin{array}{r}
\frac{\partial \varphi}{\partial x_{2}} \\
-\frac{\partial \varphi}{\partial x_{1}}
\end{array}\right) \quad \text { and } \quad \operatorname{curl}(\boldsymbol{v}):=\frac{\partial v_{2}}{\partial x_{1}}-\frac{\partial v_{1}}{\partial x_{2}}
$$

Next, letting $\left(\boldsymbol{u}_{h},\left(p_{h}, \lambda_{h}\right)\right) \in \boldsymbol{H}_{h} \times \boldsymbol{Q}_{h}$ be the unique solution of (4.1), we denote

$$
\mathbf{r}\left(\boldsymbol{u}_{h}, p_{h} ; \boldsymbol{f}\right):=\gamma \boldsymbol{f}+\gamma p_{h} \boldsymbol{f}-\alpha_{0} \gamma \boldsymbol{u}_{h} \quad \text { in } \quad \Omega,
$$

and define for each $T \in \mathcal{T}_{h}$ the a posteriori error indicator:

$$
\begin{aligned}
\theta_{T}^{2}:= & \left\|\operatorname{div} \boldsymbol{u}_{h}\right\|_{0, T}^{2}+h_{T}^{2}\left\|\mathbf{r}\left(\boldsymbol{u}_{h}, p_{h} ; \boldsymbol{f}\right)\right\|_{0, T}^{2}+h_{T}^{2}\left\|\operatorname{curl}\left\{\mathbf{r}\left(\boldsymbol{u}_{h}, p_{h} ; \boldsymbol{f}\right)\right\}\right\|_{0, T}^{2} \\
& +\sum_{e \in \mathcal{E}(T) \cap \mathcal{E}_{h}(\Omega)} h_{e}\left\|\left[\mathbf{r}\left(\boldsymbol{u}_{h}, p_{h} ; \boldsymbol{f}\right) \cdot \boldsymbol{s}\right]\right\|_{0, e}^{2}+\sum_{e \in \mathcal{E}(T) \cap \mathcal{E}_{h}\left(\Gamma_{N}\right)} h_{e}\left\|\mathbf{r}\left(\boldsymbol{u}_{h}, p_{h} ; \boldsymbol{f}\right) \cdot \boldsymbol{s}-\frac{d \lambda_{h}}{d \boldsymbol{s}}\right\|_{0, e}^{2} \\
& +\sum_{e \in \mathcal{E}(T) \cap \mathcal{E}_{h}\left(\Gamma_{N}\right)} h_{e}\left\{\left\|\lambda_{h}+p_{h}\right\|_{0, e}^{2}+\left\|g-\boldsymbol{u}_{h} \cdot \boldsymbol{\nu}\right\|_{0, e}^{2}\right\} \\
& +\sum_{e \in \mathcal{E}(T) \cap \mathcal{E}_{h}\left(\Gamma_{D}\right)} h_{e}\left\|\mathbf{r}\left(\boldsymbol{u}_{h}, p_{h} ; \boldsymbol{f}\right) \cdot \boldsymbol{s}\right\|_{0, e}^{2}
\end{aligned}
$$

and introduce the global a posteriori error estimator

$$
\boldsymbol{\theta}:=\left\{\sum_{T \in \mathcal{T}_{h}} \theta_{T}^{2}\right\}^{1 / 2}
$$

Note here that the inclusion of the expression $\left\|g-\boldsymbol{u}_{h} \cdot \boldsymbol{\nu}\right\|_{0, e}^{2}$ in the definition of $\theta_{T}^{2}$ requires the Neumann datum to be smoother than $H_{00}^{-1 / 2}\left(\Gamma_{N}\right)$, namely $g \in L^{2}\left(\Gamma_{N}\right)$.

Then, the following theorem constitutes the main result of this section.

Theorem 5.1. Assume that $\boldsymbol{f} \in \boldsymbol{L}^{\infty}(\Omega), g \in L^{2}\left(\Gamma_{N}\right)$, and that they are piecewise polynomials on $\mathcal{T}_{h}$ and $\Gamma_{N, h}$, respectively, for each $h>0$. Let $(\boldsymbol{u},(p, \lambda)) \in \boldsymbol{H} \times \boldsymbol{Q}$ and $\left(\boldsymbol{u}_{h},\left(p_{h}, \lambda_{h}\right)\right) \in \boldsymbol{H}_{h} \times \boldsymbol{Q}_{h}$ be the unique solutions of (3.1) and (4.1), respectively. Then, there exist constants $C_{\mathrm{rel}}>0$ and $C_{\mathrm{eff}}>0$, independent of $h$, such that

$$
C_{\text {eff }} \boldsymbol{\theta} \leq\left\|(\boldsymbol{u},(p, \lambda))-\left(\boldsymbol{u}_{h},\left(p_{h}, \lambda_{h}\right)\right)\right\|_{\boldsymbol{H} \times \boldsymbol{Q}} \leq C_{\mathrm{rel}} \boldsymbol{\theta} .
$$

We remark that when $f$ and $g$ are not both piecewise polynomials, then additional higher order terms (h.o.t.) arising from suitable polynomial approximations of these functions will appear in (5.3). Now, the reliability and efficiency estimates, that is the upper and lower bounds in (5.3), are derived below in Sections 5.2 and 5.3, respectively. To this end, we first provide some preliminary results.

\subsection{Preliminary results}

In this section we provide several useful results concerning the Clément and Raviart-Thomas interpolation operators, and the Helmholtz decomposition of vector fields in $\boldsymbol{H}$ (div; $\Omega$ ). 


\subsubsection{Clément interpolator}

Let $I_{h}: H^{1}(\Omega) \rightarrow X_{h}$ be the Clément interpolation operator (cf. [20]), where

$$
X_{h}:=\left\{\varphi_{h} \in C(\bar{\Omega}):\left.\quad \varphi_{h}\right|_{T} \in P_{1}(T) \quad \forall T \in \mathcal{T}_{h}\right\} .
$$

The local approximation properties of $I_{h}$ are summarized in the following lemma.

Lemma 5.2. There exist $c_{1}, c_{2}>0$, independent of $h$, such that for all $\varphi \in H^{1}(\Omega)$ there holds

$$
\left\|\varphi-I_{h}(\varphi)\right\|_{0, T} \leq c_{1} h_{T}\|\varphi\|_{1, \Delta(T)} \quad \forall T \in \mathcal{T}_{h}
$$

and

$$
\left\|\varphi-I_{h}(\varphi)\right\|_{0, e} \leq c_{2} h_{e}^{1 / 2}\|\varphi\|_{1, \Delta(e)} \quad \forall e \in \mathcal{E}_{h}(\Omega) \cup \mathcal{E}_{h}(\Gamma),
$$

where $\Delta(T):=\cup\left\{T^{\prime} \in \mathcal{T}_{h}: \quad T^{\prime} \cap T \neq \emptyset\right\}$ and $\Delta(e):=\cup\left\{T^{\prime} \in \mathcal{T}_{h}: \quad T^{\prime} \cap e \neq \emptyset\right\}$.

Proof. See [20].

\subsubsection{Helmholtz decomposition}

Lemma 5.3. For each $\boldsymbol{v} \in \boldsymbol{H}(\operatorname{div} ; \Omega)$ there exist $\boldsymbol{\zeta} \in \boldsymbol{H}^{1}(\Omega)$ and $\varphi \in H^{1}(\Omega)$, with $\int_{\Omega} \varphi=0$, such that $\boldsymbol{v}=\boldsymbol{\zeta}+\operatorname{curl} \varphi$ in $\Omega$ and

$$
\|\boldsymbol{\zeta}\|_{1, \Omega}+\|\varphi\|_{1, \Omega} \leq C\|\boldsymbol{v}\|_{\operatorname{div}, \Omega},
$$

where $C$ is a positive constant independent of $\boldsymbol{v}$.

Proof. We proceed as in [24, Lemma 3.4] (see also [29, Section 3.2.2]) by introducing first a convex domain $\widetilde{\Omega}$ containing $\bar{\Omega}$. Then, given $\boldsymbol{v} \in \boldsymbol{H}(\operatorname{div} ; \Omega)$, we define $q \in L^{2}(\widetilde{\Omega})$ by

$$
q:=\left\{\begin{array}{lll}
\operatorname{div} \boldsymbol{v} & \text { in } & \Omega \\
0 & \text { in } & \widetilde{\Omega} \backslash \bar{\Omega}
\end{array} .\right.
$$

and let $z \in H_{0}^{1}(\widetilde{\Omega})$ be the unique solution of the boundary value problem:

$$
\Delta z=q \text { in } \widetilde{\Omega}, \quad z=0 \text { on } \partial \widetilde{\Omega} .
$$

The elliptic regularity result for (5.5) guarantees that actually $z \in H^{2}(\widetilde{\Omega})$ and

$$
\|z\|_{2, \widetilde{\Omega}} \leq C\|q\|_{0, \widetilde{\Omega}}=\|\operatorname{div} \boldsymbol{v}\|_{0, \Omega} .
$$

It follows that $\boldsymbol{\zeta}:=\left.\nabla z\right|_{\Omega}$ belongs to $\boldsymbol{H}^{1}(\Omega)$,

$$
\operatorname{div} \boldsymbol{\zeta}=\operatorname{div} \boldsymbol{v} \text { in } \Omega,
$$

and

$$
\|\boldsymbol{\zeta}\|_{1, \Omega} \leq C\|z\|_{2, \Omega} \leq C\|\operatorname{div} \boldsymbol{v}\|_{0, \Omega} .
$$

Next, since $\operatorname{div}(\boldsymbol{v}-\boldsymbol{\zeta})=0$ in $\Omega$, and $\Omega$ is connected, there exists (cf. [34, Theorem 3.1]) $\varphi \in H^{1}(\Omega)$, with $\int_{\Omega} \varphi=0$, such that $\boldsymbol{v}-\boldsymbol{\zeta}=\operatorname{curl} \varphi$. Note that this identity, combined with the equivalence between $\|\varphi\|_{1, \Omega}$ and $|\varphi|_{1, \Omega}$, and (5.6) imply that

$$
\|\varphi\|_{1, \Omega} \leq C|\varphi|_{1, \Omega}=C\|\boldsymbol{v}-\boldsymbol{\zeta}\|_{0, \Omega} \leq C\left\{\|\boldsymbol{v}\|_{0, \Omega}+\|\boldsymbol{\zeta}\|_{0, \Omega}\right\} \leq C\|\boldsymbol{v}\|_{\operatorname{div}, \Omega},
$$

which, together with (5.6) again, yields (5.4). 


\subsubsection{Raviart-Thomas interpolator}

Let $\Pi_{h}: \boldsymbol{H}^{1}(\Omega) \rightarrow \boldsymbol{H}_{h}$ be the usual Raviart-Thomas interpolation operator, which is characterized by the identity

$$
\int_{e} \Pi_{h}(\boldsymbol{w}) \cdot \boldsymbol{\nu}=\int_{e} \boldsymbol{w} \cdot \boldsymbol{\nu} \quad \forall e \in \mathcal{E}_{h}, \quad \forall \boldsymbol{w} \in \boldsymbol{H}^{1}(\Omega)
$$

It is easy to show, using (5.7), that

$$
\operatorname{div}\left(\Pi_{h}(\boldsymbol{w})\right)=\mathcal{P}_{h}(\operatorname{div} \boldsymbol{w}) \quad \forall \boldsymbol{w} \in \boldsymbol{H}^{1}(\Omega),
$$

where $\mathcal{P}_{h}$ is the $L^{2}(\Omega)$-orthogonal projector onto $Q_{h}^{p}$ (cf. (4.10)).

Lemma 5.4. $\Pi_{h}$ satisfies the following approximation properties

$$
\left\|\boldsymbol{w}-\Pi_{h}(\boldsymbol{w})\right\|_{0, T} \leq C h_{T}\|\boldsymbol{w}\|_{1, T} \quad \forall T \in \mathcal{T}_{h}, \quad \forall \boldsymbol{w} \in \boldsymbol{H}^{1}(\Omega),
$$

and

$$
\left\|\left(\boldsymbol{w}-\Pi_{h}(\boldsymbol{w})\right) \cdot \boldsymbol{\nu}\right\|_{0, e} \leq C h_{e}^{1 / 2}\|\boldsymbol{w}\|_{1, T_{e}} \quad \forall e \in \mathcal{E}_{h} \cap \partial T_{e}, \quad \forall \boldsymbol{w} \in \boldsymbol{H}^{1}(\Omega),
$$

where $T_{e}$ in (5.10) is a triangle of $\mathcal{T}_{h}$ containing e on its boundary.

Proof. See, e.g. [15], [27, Lemmas 3.16 and 3.18], and [49].

\subsection{Reliability of the a posteriori error estimator}

We first rely on our continuous variational formulation (rewritten as (3.3) - (3.4)) and the associated

continuous dependence estimate (3.20). In fact, assuming certainly that $\|\boldsymbol{f}\|_{\infty, \Omega} \leq \frac{1}{2 \tilde{C} \gamma}$, it is easy to show that (3.20) is equivalent to the global inf-sup condition

$$
\frac{1}{4 \tilde{C}}\|(\boldsymbol{w},(r, \eta))\|_{\boldsymbol{H} \times \boldsymbol{Q}} \leq \sup _{\substack{(\boldsymbol{v},(q, \xi)) \in \boldsymbol{H} \times \boldsymbol{Q} \\(\boldsymbol{v},(q, \xi)) \neq 0}}\left\{\frac{\boldsymbol{a}(\boldsymbol{w}, \boldsymbol{v})+\boldsymbol{b}_{1}(\boldsymbol{v},(r, \eta))+\boldsymbol{b}_{2}(\boldsymbol{w},(q, \xi))}{\|(\boldsymbol{v},(q, \xi))\|_{\boldsymbol{H} \times \boldsymbol{Q}}}\right\},
$$

for all $(\boldsymbol{w},(r, \eta)) \in \boldsymbol{H} \times \boldsymbol{Q}$. Hence, we have the following preliminary estimate for the error.

Lemma 5.5. Let $(\boldsymbol{u},(p, \lambda)) \in \boldsymbol{H} \times \boldsymbol{Q}$ and $\left(\boldsymbol{u}_{h},\left(p_{h}, \lambda_{h}\right)\right) \in \boldsymbol{H}_{h} \times \boldsymbol{Q}_{h}$ be the unique solutions of (3.1) and (4.1), respectively. Then, there exists a constant $C>0$, independent of $h$, such that

$$
\left\|(\boldsymbol{u},(p, \lambda))-\left(\boldsymbol{u}_{h},\left(p_{h}, \lambda_{h}\right)\right)\right\|_{\boldsymbol{H} \times \boldsymbol{Q}} \leq C\left\{\|\boldsymbol{E}\|_{\boldsymbol{H}^{\prime}}+\left\|g-\boldsymbol{u}_{h} \cdot \boldsymbol{\nu}\right\|_{0 ;-1 / 2, \Gamma_{N}}+\left\|\operatorname{div} \boldsymbol{u}_{h}\right\|_{0, \Omega}\right\},
$$

where $\boldsymbol{E} \in \boldsymbol{H}^{\prime}$ is an operator defined by

$$
\boldsymbol{E}(\boldsymbol{v}):=\boldsymbol{F}(\boldsymbol{v})-\boldsymbol{a}\left(\boldsymbol{u}_{h}, \boldsymbol{v}\right)-\boldsymbol{b}_{1}\left(\boldsymbol{v},\left(p_{h}, \lambda_{h}\right)\right) \quad \forall \boldsymbol{v} \in \boldsymbol{H},
$$

and satisfies $\boldsymbol{E}\left(\boldsymbol{v}_{h}\right)=0 \quad \forall \boldsymbol{v}_{h} \in \boldsymbol{H}_{h}$.

Proof. Applying (5.11) to the error $(\boldsymbol{w},(r, \eta)):=\left(\boldsymbol{u},\left(p, \lambda_{h}\right)\right)-\left(\boldsymbol{u}_{h},\left(p_{h}, \lambda_{h}\right)\right)$, we arrive at

$$
\begin{aligned}
& \frac{1}{4 \tilde{C}}\left\|(\boldsymbol{u},(p, \lambda))-\left(\boldsymbol{u}_{h},\left(p_{h}, \lambda_{h}\right)\right)\right\|_{\boldsymbol{H} \times Q} \\
& \leq \sup _{\substack{(\boldsymbol{v}, q) \in \boldsymbol{H} \times Q \\
(\boldsymbol{v}, q) \neq 0}}\left\{\frac{\boldsymbol{a}\left(\boldsymbol{u}-\boldsymbol{u}_{h}, \boldsymbol{v}\right)+\boldsymbol{b}_{1}\left(\boldsymbol{v},(p, \lambda)-\left(p_{h}, \lambda_{h}\right)\right)+\boldsymbol{b}_{2}\left(\boldsymbol{u}-\boldsymbol{u}_{h},(q, \xi)\right)}{\|(\boldsymbol{v},(q, \xi))\|_{\boldsymbol{H} \times \boldsymbol{Q}}}\right\},
\end{aligned}
$$


that is

$$
\frac{1}{4 \tilde{C}}\left\|(\boldsymbol{u},(p, \lambda))-\left(\boldsymbol{u}_{h},\left(p_{h}, \lambda_{h}\right)\right)\right\|_{\boldsymbol{H} \times \boldsymbol{Q}} \leq \sup _{\substack{(\boldsymbol{v},(q, \xi)) \in \boldsymbol{H} \times \boldsymbol{Q} \\(\boldsymbol{v},(q, \xi)) \neq 0}}\left\{\frac{\boldsymbol{E}(\boldsymbol{v})+\boldsymbol{b}_{2}\left(\boldsymbol{u}-\boldsymbol{u}_{h},(q, \xi)\right)}{\|(\boldsymbol{v},(q, \xi))\|_{\boldsymbol{H} \times \boldsymbol{Q}}}\right\},
$$

where

$$
\boldsymbol{E}(\boldsymbol{v}):=\boldsymbol{a}\left(\boldsymbol{u}-\boldsymbol{u}_{h}, \boldsymbol{v}\right)+\boldsymbol{b}_{1}\left(\boldsymbol{v},(p, \lambda)-\left(p_{h}, \lambda_{h}\right)\right) \quad \forall \boldsymbol{v} \in \boldsymbol{H} .
$$

Note here, according to the first equation of (3.3), that the above expression defining $\boldsymbol{E}$ coincides with the one given in (5.12). Next, taking into account that

$$
\frac{|\boldsymbol{E}(\boldsymbol{v})|}{\|(\boldsymbol{v}, q)\|} \leq \frac{|\boldsymbol{E}(\boldsymbol{v})|}{\|\boldsymbol{v}\|} \quad \text { and } \quad \frac{\left|\boldsymbol{b}_{2}\left(\boldsymbol{u}-\boldsymbol{u}_{h},(q, \xi)\right)\right|}{\|(\boldsymbol{v},(q, \xi))\|} \leq \frac{\left|\boldsymbol{b}_{2}\left(\boldsymbol{u}-\boldsymbol{u}_{h},(q, \xi)\right)\right|}{\|(q, \xi)\|}
$$

we apply the supremum in (5.13) and arrive at

$$
\frac{1}{4 \tilde{C}}\left\|(\boldsymbol{u},(p, \lambda))-\left(\boldsymbol{u}_{h},\left(p_{h}, \lambda_{h}\right)\right)\right\|_{\boldsymbol{H} \times \boldsymbol{Q}} \leq\|\boldsymbol{E}\|_{\boldsymbol{H}^{\prime}}+\left\|\boldsymbol{b}_{2}\left(\boldsymbol{u}-\boldsymbol{u}_{h}, \cdot\right)\right\|_{\boldsymbol{Q}^{\prime}}
$$

Now, from the definition of $\boldsymbol{b}_{2}=\boldsymbol{b}$ and the second equation of (3.3), we find that

$$
\boldsymbol{b}_{2}\left(\boldsymbol{u}-\boldsymbol{u}_{h},(q, \xi)\right)=\boldsymbol{b}_{2}(\boldsymbol{u},(q, \xi))-\boldsymbol{b}_{2}\left(\boldsymbol{u}_{h},(q, \xi)\right)=\left\langle g-\boldsymbol{u}_{h} \cdot \boldsymbol{\nu}, \xi\right\rangle_{\Gamma_{N}}-\int_{\Omega} q \operatorname{div} \boldsymbol{u}_{h},
$$

and therefore

$$
\left\|\boldsymbol{b}_{2}\left(\boldsymbol{u}-\boldsymbol{u}_{h}, \cdot\right)\right\|_{\boldsymbol{Q}^{\prime}} \leq\left\|g-\boldsymbol{u}_{h} \cdot \boldsymbol{\nu}\right\|_{0 ;-1 / 2, \Gamma_{N}}+\left\|\operatorname{div} \boldsymbol{u}_{h}\right\|_{0, \Omega} .
$$

Finally, it is clear from the first equation of (4.1) that $\boldsymbol{E}\left(\boldsymbol{v}_{h}\right)=0 \quad \forall \boldsymbol{v}_{h} \in \boldsymbol{H}_{h}$, which finishes the proof.

In order to complete the derivation of the a posteriori error estimate, we need to obtain suitable upper bounds for $\|\boldsymbol{E}\|_{\boldsymbol{H}^{\prime}}$ and for the Neumann residual $\left\|g-\boldsymbol{u}_{h} \cdot \boldsymbol{\nu}\right\|_{0 ;-1 / 2, \Gamma_{N}}$. We proceed first with the norm of the functional $\boldsymbol{E}$, for which we make use in what follows of the Helmholtz decomposition and the Clément and Raviart-Thomas interpolation operators introduced in Section 5.1 . More precisely, given $\boldsymbol{v} \in \boldsymbol{H}$, we know from Lemma 5.3 that there exist $\boldsymbol{\zeta} \in \boldsymbol{H}^{1}(\Omega)$ and $\varphi \in H^{1}(\Omega)$, such that $\boldsymbol{v}=\boldsymbol{\zeta}+\operatorname{curl} \varphi$ in $\Omega$ and

$$
\|\boldsymbol{\zeta}\|_{1, \Omega}+\|\varphi\|_{1, \Omega} \leq C\|\boldsymbol{v}\|_{\operatorname{div}, \Omega} .
$$

Hence, we let $\varphi_{h}:=I_{h}(\varphi)$ and introduce what we call the discrete Helmholtz decomposition

$$
\boldsymbol{v}_{h}:=\Pi_{h}(\boldsymbol{\zeta})+\operatorname{curl} \varphi_{h}
$$

which clearly belongs to $\boldsymbol{H}_{h}$. In this way, and recalling from Lemma 5.5 that $\boldsymbol{E}\left(\boldsymbol{v}_{h}\right)=0$, we can write

$$
\boldsymbol{E}(\boldsymbol{v})=\boldsymbol{E}\left(\boldsymbol{v}-\boldsymbol{v}_{h}\right)=\boldsymbol{E}\left(\boldsymbol{\zeta}-\Pi_{h}(\boldsymbol{\zeta})\right)+\boldsymbol{E}\left(\operatorname{curl}\left(\varphi-\varphi_{h}\right)\right),
$$

where, according to (5.12), the definitions of $\boldsymbol{F}, \boldsymbol{a}$, and $\boldsymbol{b}$ (cf. (3.5) and (3.2)), and (5.1), we have

$$
\boldsymbol{E}(\boldsymbol{w})=\int_{\Omega} \mathbf{r}\left(\boldsymbol{u}_{h}, p_{h} ; \boldsymbol{f}\right) \cdot \boldsymbol{w}-\int_{\Omega} p_{h} \operatorname{div} \boldsymbol{w}-\left\langle\boldsymbol{w} \cdot \boldsymbol{\nu}, \lambda_{h}\right\rangle_{\Gamma_{N}} \quad \forall \boldsymbol{w} \in \boldsymbol{H} .
$$


Hence, using (5.8), the fact that $\left.p_{h}\right|_{e} \in P_{0}(e)$ for each $e \in \mathcal{E}_{h}\left(\Gamma_{N}\right)$, (5.7), and the obvious identity $\operatorname{div}\left\{\operatorname{curl}\left(\varphi-\varphi_{h}\right)\right\}=0$, we deduce from (5.16) that

$$
\boldsymbol{E}\left(\boldsymbol{\zeta}-\Pi_{h}(\boldsymbol{\zeta})\right)=\int_{\Omega} \mathbf{r}\left(\boldsymbol{u}_{h}, p_{h} ; \boldsymbol{f}\right) \cdot\left(\boldsymbol{\zeta}-\Pi_{h}(\boldsymbol{\zeta})\right)-\left\langle\left(\boldsymbol{\zeta}-\Pi_{h}(\boldsymbol{\zeta})\right) \cdot \boldsymbol{\nu}, \lambda_{h}+p_{h}\right\rangle_{\Gamma_{N}}
$$

and

$$
\boldsymbol{E}\left(\operatorname{curl}\left(\varphi-\varphi_{h}\right)\right)=\int_{\Omega} \mathbf{r}\left(\boldsymbol{u}_{h}, p_{h} ; \boldsymbol{f}\right) \cdot \operatorname{curl}\left(\varphi-\varphi_{h}\right)-\left\langle\left(\operatorname{curl}\left(\varphi-\varphi_{h}\right)\right) \cdot \boldsymbol{\nu}, \lambda_{h}\right\rangle_{\Gamma_{N}}
$$

Consequently, in order to estimate $|\boldsymbol{E}(\boldsymbol{v})|$ in terms of residual terms and $\|\boldsymbol{v}\|_{\boldsymbol{H}}$, we now proceed to get suitable upper bounds for each one of the above two expressions. We begin with the following lemma.

Lemma 5.6. There exists $C>0$, independent of $h$, such that

$$
\left|\boldsymbol{E}\left(\boldsymbol{\zeta}-\Pi_{h}(\boldsymbol{\zeta})\right)\right| \leq C\left\{\sum_{T \in \mathcal{T}_{h}} \widetilde{\theta}_{T}^{2}\right\}^{1 / 2}\|\boldsymbol{v}\|_{\boldsymbol{H}}
$$

where

$$
\widetilde{\theta}_{T}^{2}:=h_{T}^{2}\left\|\mathbf{r}\left(\boldsymbol{u}_{h}, p_{h} ; \boldsymbol{f}\right)\right\|_{0, T}^{2}+\sum_{e \in \mathcal{E}(T) \cap \mathcal{E}_{h}\left(\Gamma_{N}\right)} h_{e}\left\|\lambda_{h}+p_{h}\right\|_{0, e}^{2} .
$$

Proof. We first notice that the fact that $\boldsymbol{\zeta} \in \boldsymbol{H}^{1}(\Omega)$ guarantees that $\left(\boldsymbol{\zeta}-\Pi_{h}(\boldsymbol{\zeta})\right) \in \boldsymbol{L}^{2}\left(\Gamma_{N}\right)$, and then

$$
\left\langle\left(\boldsymbol{\zeta}-\Pi_{h}(\boldsymbol{\zeta})\right) \cdot \boldsymbol{\nu}, \lambda_{h}+p_{h}\right\rangle_{\Gamma_{N}}=\sum_{e \in \mathcal{E}_{h}\left(\Gamma_{N}\right)} \int_{e}\left(\boldsymbol{\zeta}-\Pi_{h}(\boldsymbol{\zeta})\right) \cdot \boldsymbol{\nu}\left(\lambda_{h}+p_{h}\right)
$$

which, together with (5.17), gives

$$
\boldsymbol{E}\left(\boldsymbol{\zeta}-\Pi_{h}(\boldsymbol{\zeta})\right)=\sum_{T \in \mathcal{T}_{h}} \int_{T} \mathbf{r}\left(\boldsymbol{u}_{h}, p_{h} ; \boldsymbol{f}\right) \cdot\left(\boldsymbol{\zeta}-\Pi_{h}(\boldsymbol{\zeta})\right)-\sum_{e \in \mathcal{E}_{h}\left(\Gamma_{N}\right)} \int_{e}\left(\boldsymbol{\zeta}-\Pi_{h}(\boldsymbol{\zeta})\right) \cdot \boldsymbol{\nu}\left(\lambda_{h}+p_{h}\right) .
$$

In this way, employing the Cauchy-Schwarz inequality, the approximation properties (5.9) and (5.10), and then the estimate (5.14), we deduce from the above expression that

$$
\begin{gathered}
\left|\boldsymbol{E}\left(\boldsymbol{\zeta}-\Pi_{h}(\boldsymbol{\zeta})\right)\right| \leq C\left\{\sum_{T \in \mathcal{T}_{h}} h_{T}\left\|\mathbf{r}\left(\boldsymbol{u}_{h}, p_{h} ; \boldsymbol{f}\right)\right\|_{0, T}\|\boldsymbol{\zeta}\|_{1, T}+\sum_{e \in \mathcal{E}_{h}\left(\Gamma_{N}\right)} h_{e}^{1 / 2}\left\|\lambda_{h}+p_{h}\right\|_{0, e}\|\boldsymbol{\zeta}\|_{1, T_{e}}\right\} \\
\leq C\left\{\sum_{T \in \mathcal{T}_{h}} h_{T}^{2}\left\|\mathbf{r}\left(\boldsymbol{u}_{h}, p_{h} ; \boldsymbol{f}\right)\right\|_{0, T}^{2}+\sum_{e \in \mathcal{E}_{h}\left(\Gamma_{N}\right)} h_{e}\left\|\lambda_{h}+p_{h}\right\|_{0, e}^{2}\right\}^{1 / 2}\|\boldsymbol{v}\|_{\boldsymbol{H}},
\end{gathered}
$$

which yields (5.19) - (5.20) and completes the proof.

The bound for $\left|\boldsymbol{E}\left(\operatorname{curl}\left(\varphi-\varphi_{h}\right)\right)\right|($ cf. (5.18)) is provided next. 
Lemma 5.7. There exists $C>0$, independent of $h$, such that

$$
\left|\boldsymbol{E}\left(\operatorname{curl}\left(\varphi-\varphi_{h}\right)\right)\right| \leq C\left\{\sum_{T \in \mathcal{T}_{h}} \widehat{\theta}_{T}^{2}\right\}^{1 / 2}\|\boldsymbol{v}\|_{\operatorname{div}, \Omega}
$$

where

$$
\begin{gathered}
\widehat{\theta}_{T}^{2}:=h_{T}^{2}\left\|\operatorname{curl}\left\{\mathbf{r}\left(\boldsymbol{u}_{h}, p_{h} ; \boldsymbol{f}\right)\right\}\right\|_{0, T}^{2}+\sum_{e \in \mathcal{E}(T) \cap \mathcal{E}_{h}(\Omega)} h_{e}\left\|\left[\mathbf{r}\left(\boldsymbol{u}_{h}, p_{h} ; \boldsymbol{f}\right) \cdot \boldsymbol{s}\right]\right\|_{0, e}^{2} \\
+\sum_{e \in \mathcal{E}(T) \cap \mathcal{E}_{h}\left(\Gamma_{N}\right)} h_{e}\left\|\mathbf{r}\left(\boldsymbol{u}_{h}, p_{h} ; \boldsymbol{f}\right) \cdot \boldsymbol{s}-\frac{d \lambda_{h}}{d \boldsymbol{s}}\right\|_{0, e}^{2}+\sum_{e \in \mathcal{E}(T) \cap \mathcal{E}_{h}\left(\Gamma_{D}\right)} h_{e}\left\|\mathbf{r}\left(\boldsymbol{u}_{h}, p_{h} ; \boldsymbol{f}\right) \cdot \boldsymbol{s}\right\|_{0, e}^{2} .
\end{gathered}
$$

Proof. Since

$$
\operatorname{curl}\left(\varphi-\varphi_{h}\right) \cdot \boldsymbol{\nu}=-\frac{d}{d \boldsymbol{s}}\left(\varphi-\varphi_{h}\right) \quad \text { and } \quad \frac{d \lambda_{h}}{d \boldsymbol{s}} \in L^{2}\left(\Gamma_{N}\right)
$$

we find, integrating by parts and using that $\lambda_{h} \in H_{00}^{1 / 2}\left(\Gamma_{N}\right)$, that

$$
\left\langle\operatorname{curl}\left(\varphi-\varphi_{h}\right) \cdot \nu, \lambda_{h}\right\rangle_{\Gamma_{N}}=-\left\langle\frac{d}{d s}\left(\varphi-\varphi_{h}\right), \lambda_{h}\right\rangle_{\Gamma_{N}}=\int_{\Gamma_{N}} \frac{d \lambda_{h}}{d s}\left(\varphi-\varphi_{h}\right),
$$

and hence, we get from (5.18) that

$$
\boldsymbol{E}\left(\operatorname{curl}\left(\varphi-\varphi_{h}\right)\right)=\int_{\Omega} \mathbf{r}\left(\boldsymbol{u}_{h}, p_{h} ; \boldsymbol{f}\right) \cdot \operatorname{curl}\left(\varphi-\varphi_{h}\right)-\sum_{e \in \mathcal{E}_{h}\left(\Gamma_{N}\right)} \int_{e} \frac{d \lambda_{h}}{d \boldsymbol{s}}\left(\varphi-\varphi_{h}\right)
$$

Now, integrating by parts on each $T \in \mathcal{T}_{h}$, we obtain

$$
\begin{aligned}
\int_{\Omega} \mathbf{r}\left(\boldsymbol{u}_{h}, p_{h} ; \boldsymbol{f}\right) \cdot \operatorname{curl}\left(\varphi-\varphi_{h}\right) \\
\quad=\sum_{T \in \mathcal{T}_{h}}\left\{-\int_{T} \operatorname{curl}\left\{\mathbf{r}\left(\boldsymbol{u}_{h}, p_{h} ; \boldsymbol{f}\right)\right\}\left(\varphi-\varphi_{h}\right)+\int_{\partial T} \mathbf{r}\left(\boldsymbol{u}_{h}, p_{h} ; \boldsymbol{f}\right) \cdot \boldsymbol{s}\left(\varphi-\varphi_{h}\right)\right\}
\end{aligned}
$$

which replaced back into $(5.23)$, leads to

$$
\begin{aligned}
& \boldsymbol{E}\left(\operatorname{curl}\left(\varphi-\varphi_{h}\right)\right)=-\sum_{T \in \mathcal{T}_{h}} \int_{T} \operatorname{curl}\left\{\mathbf{r}\left(\boldsymbol{u}_{h}, p_{h} ; \boldsymbol{f}\right)\right\}\left(\varphi-\varphi_{h}\right)+\sum_{e \in \mathcal{E}_{h}(\Omega)} \int_{e}\left[\mathbf{r}\left(\boldsymbol{u}_{h}, p_{h} ; \boldsymbol{f}\right) \cdot \boldsymbol{s}\right]\left(\varphi-\varphi_{h}\right) \\
& \quad+\sum_{e \in \mathcal{E}_{h}\left(\Gamma_{N}\right)} \int_{e}\left\{\mathbf{r}\left(\boldsymbol{u}_{h}, p_{h} ; \boldsymbol{f}\right) \cdot \boldsymbol{s}-\frac{d \lambda_{h}}{d \boldsymbol{s}}\right\}\left(\varphi-\varphi_{h}\right)+\sum_{e \in \mathcal{E}_{h}\left(\Gamma_{D}\right)} \int_{e} \mathbf{r}\left(\boldsymbol{u}_{h}, p_{h} ; \boldsymbol{f}\right) \cdot \boldsymbol{s}\left(\varphi-\varphi_{h}\right)
\end{aligned}
$$

Then, applying Cauchy-Schwarz inequality and the approximation properties of the Clément interpolator (cf. Lemma 5.2), and then using that the number of elements of $\Delta(T)$ is bounded independently of $T \in \mathcal{T}_{h}$, it follows that

$$
\begin{gathered}
\left|\sum_{T \in \mathcal{T}_{h}} \int_{T} \operatorname{curl}\left\{\mathbf{r}\left(\boldsymbol{u}_{h}, p_{h} ; \boldsymbol{f}\right)\right\}\left(\varphi-\varphi_{h}\right)\right| \leq c_{1} \sum_{T \in \mathcal{T}_{h}} h_{T}\left\|\operatorname{curl}\left\{\mathbf{r}\left(\boldsymbol{u}_{h}, p_{h} ; \boldsymbol{f}\right)\right\}\right\|_{0, T}\|\varphi\|_{1, \Delta(T)} \\
\leq C\left\{\sum_{T \in \mathcal{T}_{h}} h_{T}^{2}\left\|\operatorname{curl}\left\{\mathbf{r}\left(\boldsymbol{u}_{h}, p_{h} ; \boldsymbol{f}\right)\right\}\right\|_{0, T}^{2}\right\}^{1 / 2}\|\varphi\|_{1, \Omega} .
\end{gathered}
$$


In turn, proceeding similarly and taking into account now that the number of elements of $\Delta(e)$ is bounded independently of $e \in \mathcal{E}_{h}(\Omega) \cup \mathcal{E}_{h}\left(\Gamma_{D}\right) \cup \mathcal{E}_{h}\left(\Gamma_{N}\right)$, we arrive at

$$
\left.\left.\mid \sum_{e \in \mathcal{E}_{h}(\Omega)} \int_{e}\left[\mathbf{r}\left(\boldsymbol{u}_{h}, p_{h} ; \boldsymbol{f}\right)\right\} \cdot \boldsymbol{s}\right]\left(\varphi-\varphi_{h}\right) \mid \leq C\left\{\sum_{e \in \mathcal{E}_{h}(\Omega)} h_{e} \|\left[\mathbf{r}\left(\boldsymbol{u}_{h}, p_{h} ; \boldsymbol{f}\right)\right\} \cdot \boldsymbol{s}\right] \|_{0, e}^{2}\right\}^{1 / 2}\|\varphi\|_{1, \Omega},
$$

and

$$
\begin{aligned}
& \left|\sum_{e \in \mathcal{E}_{h}\left(\Gamma_{N}\right)} \int_{e}\left\{\mathbf{r}\left(\boldsymbol{u}_{h}, p_{h} ; \boldsymbol{f}\right) \cdot \boldsymbol{s}-\frac{d \lambda_{h}}{d \boldsymbol{s}}\right\}\left(\varphi-\varphi_{h}\right)\right| \\
& \leq C\left\{\sum_{e \in \mathcal{E}_{h}\left(\Gamma_{N}\right)} h_{e}\left\|\mathbf{r}\left(\boldsymbol{u}_{h}, p_{h} ; \boldsymbol{f}\right) \cdot \boldsymbol{s}-\frac{d \lambda_{h}}{d \boldsymbol{s}}\right\|_{0, e}^{2}\right\}^{1 / 2}\|\varphi\|_{1, \Omega} .
\end{aligned}
$$

Next, following basically the same arguments, we obtain

$$
\left|\sum_{e \in \mathcal{E}_{h}\left(\Gamma_{D}\right)} \int_{e} \mathbf{r}\left(\boldsymbol{u}_{h}, p_{h} ; \boldsymbol{f}\right) \cdot \boldsymbol{s}\left(\varphi-\varphi_{h}\right)\right| \leq C\left\{\sum_{e \in \mathcal{E}_{h}\left(\Gamma_{N}\right)} h_{e}\left\|\mathbf{r}\left(\boldsymbol{u}_{h}, p_{h} ; \boldsymbol{f}\right) \cdot \boldsymbol{s}\right\|_{0, e}^{2}\right\}^{1 / 2}\|\varphi\|_{1, \Omega} .
$$

Finally, combining estimates (5.24), (5.25), (5.26) and (5.27) with the bound $\|\varphi\|_{1, \Omega} \leq C\|\boldsymbol{v}\|_{\operatorname{div}, \Omega}$ (Lemma 5.3), we arrive at estimate (5.21) and conclude the proof.

As a straightforward consequence of identity (5.15) and Lemmas 5.6 and 5.7, we deduce the following upper bound for $\|\boldsymbol{E}\|_{\boldsymbol{H}^{\prime}}$.

Lemma 5.8. There exists $C>0$, independent of $h$, such that

$$
\|\boldsymbol{E}\|_{\boldsymbol{H}^{\prime}} \leq\left\{\sum_{T \in \mathcal{T}_{h}}\left\{\widetilde{\theta}_{T}^{2}+\widehat{\theta}_{T}^{2}\right\}\right\}^{1 / 2}
$$

where $\widetilde{\theta}_{T}^{2}$ and $\widehat{\theta}_{T}^{2}$ are given by (5.20) and (5.22), respectively.

In order to complete the upper bound for $\left\|(\boldsymbol{u},(p, \lambda))-\left(\boldsymbol{u}_{h},\left(p_{h}, \lambda_{h}\right)\right)\right\|_{\boldsymbol{H} \times \boldsymbol{Q}}$ provided by Lemma 5.5 , it only remains to estimate the Neumann residual $\left\|g-\boldsymbol{u}_{h} \cdot \boldsymbol{\nu}\right\|_{0 ;-1 / 2, \Gamma_{N}}$. For this purpose, we now assume that $g \in L^{2}\left(\Gamma_{N}\right)$, which certainly yields $g-\boldsymbol{u}_{h} \cdot \boldsymbol{\nu} \in L^{2}\left(\Gamma_{N}\right)$. Hence, taking $q_{h}=0$ in the second equation of (4.1), we get

$$
\left\langle g-\boldsymbol{u}_{h} \cdot \boldsymbol{\nu}, \xi_{h}\right\rangle_{\Gamma_{N}}=0 \quad \forall \xi_{h} \in Q_{h}^{\lambda},
$$

which says that $g-\boldsymbol{u}_{h} \cdot \boldsymbol{\nu}$ is $L^{2}\left(\Gamma_{N}\right)$-orthogonal to $Q_{h}^{\lambda}$ (cf. (4.13)), the continuous piecewise linear functions on the double partition $\Gamma_{N, 2 h}$ of $\Gamma_{N}$. Consequently, applying [16, Theorem 2 and eq. (1.4)] and observing that $\Gamma_{N, h}$ and $\Gamma_{N, 2 h}$ are of bounded variation (which follows from the shape-regularity of $\left\{\mathcal{T}_{h}\right\}_{h>0}$ ), we obtain

$$
\left\|g-\boldsymbol{u}_{h} \cdot \boldsymbol{\nu}\right\|_{0 ;-1 / 2, \Gamma_{N}}^{2} \leq c \sum_{e \in \mathcal{E}_{h}\left(\Gamma_{N}\right)} h_{e}\left\|g-\boldsymbol{u}_{h} \cdot \boldsymbol{\nu}\right\|_{0, e}^{2} .
$$

Finally, our reliability estimate for $\boldsymbol{\theta}$ (cf. upper bound in (5.3)) follows straightforwardly from Lemmas 5.5 and 5.8, and (5.28). 


\subsection{Efficiency of the a posteriori error estimator}

In this section we show the efficiency of our a posteriori error estimator $\boldsymbol{\theta}$. In other words, we provide upper bounds depending on the actual errors for the eight terms defining the local indicator $\theta_{T}^{2}$ (cf. (5.2)). The easiest one is the first term, for which, thanks to the incompressibility condition $\operatorname{div} \boldsymbol{u}=0$ in $\Omega$ (cf. Theorem 3.2), there holds

$$
\left\|\operatorname{div} \boldsymbol{u}_{h}\right\|_{0, T}=\left\|\operatorname{div}\left(\boldsymbol{u}_{h}-\boldsymbol{u}\right)\right\|_{0, T} \leq\left\|\boldsymbol{u}-\boldsymbol{u}_{h}\right\|_{\operatorname{div}, T} .
$$

The derivation of the corresponding upper bounds for the remaining terms in (5.2) is performed next. To this end, we proceed as in [17] and [28], and apply the localization technique based on triangle-bubble and edge-bubble functions, together with extension operators, discrete trace and inverse inequalities. Therefore, we now introduce further notations and preliminary results. Given $T \in \mathcal{T}_{h}$ and $e \in \mathcal{E}(T)$, we let $\psi_{T}$ and $\psi_{e}$ be the usual triangle-bubble and edge-bubble functions, respectively (see [54, eqs. (1.5) and (1.6)]), which satisfy:

ii) $\psi_{T} \in P_{3}(T), \psi_{T}=0$ on $\partial T, \operatorname{supp}\left(\psi_{T}\right) \subseteq T$, and $0 \leq \psi_{T} \leq 1$ in $T$.

ii) $\left.\psi_{e}\right|_{T} \in P_{2}(T), \psi_{e}=0$ on $\partial T \backslash e, \operatorname{supp}\left(\psi_{e}\right) \subseteq w_{e}:=\cup\left\{T^{\prime} \in \mathcal{T}_{h}: e \in \mathcal{E}\left(T^{\prime}\right)\right\}$, and $0 \leq \psi_{e} \leq 1$ in $w_{e}$.

We also know from [53] that, given $k \in \mathbb{N} \cup\{0\}$, there exists an extension operator $L: C(e) \rightarrow C(T)$ that satisfies $L(p) \in P_{k}(T)$ and $\left.L(p)\right|_{e}=p$ for all $p \in P_{k}(e)$. Additional properties of $\psi_{T}, \psi_{e}$ and $L$ are collected in the following lemma.

Lemma 5.9. Given $k \in \mathbb{N} \cup\{0\}$, there exist positive constants $c_{1}, c_{2}$, and $c_{3}$, depending only on $k$ and the shape regularity of the triangulations (minimum angle condition), such that for each $T \in \mathcal{T}_{h}$ and $e \in \mathcal{E}(T)$, there hold

$$
\begin{array}{cl}
\|q\|_{0, T}^{2} \leq c_{1}\left\|\psi_{T}^{1 / 2} q\right\|_{0, T}^{2} & \forall q \in P_{k}(T) \\
\|p\|_{0, e}^{2} \leq c_{2}\left\|\psi_{e}^{1 / 2} p\right\|_{0, e}^{2} & \forall p \in P_{k}(e)
\end{array}
$$

and

$$
\left\|\psi_{e}^{1 / 2} L(p)\right\|_{0, T}^{2} \leq c_{3} h_{e}\|p\|_{0, e}^{2} \quad \forall p \in P_{k}(e)
$$

Proof. See [53, Lemma 4.1].

The following inverse and discrete trace inequalities are also employed.

Lemma 5.10. Let $k, l, m \in \mathbb{N} \cup\{0\}$ such that $l \leq m$. Then there exists $c>0$, depending only on $k$, $l, m$ and the shape regularity of the triangulations, such that for each $T \in \mathcal{T}_{h}$ there holds

$$
|q|_{m, T} \leq c h_{T}^{l-m}|q|_{l, T} \quad \forall q \in P_{k}(T) .
$$

Proof. See [19, Theorem 3.2.6].

Lemma 5.11. There exists $C>0$, depending only on the shape regularity of the triangulations, such that for each $T \in \mathcal{T}_{h}$ and $e \in \mathcal{E}(T)$, there holds

$$
\|v\|_{0, e}^{2} \leq C\left\{h_{e}^{-1}\|v\|_{0, T}^{2}+h_{e}|v|_{1, T}^{2}\right\} \quad \forall v \in H^{1}(T) .
$$


Proof. See [1, Theorem 3.10] or [5, eq. (2.4)].

In turn, the following lemma, whose proof makes use of Lemmas 5.9 and 5.10, will be required for the terms involving $\operatorname{curl}\left\{\mathbf{r}\left(\boldsymbol{u}_{h}, p_{h} ; \boldsymbol{f}\right)\right\}$ and the tangential jumps of $\mathbf{r}\left(\boldsymbol{u}_{h}, p_{h} ; \boldsymbol{f}\right)$ across the edges of $\mathcal{T}_{h}$.

Lemma 5.12. Let $\boldsymbol{\rho}_{h} \in \boldsymbol{L}^{2}(\Omega)$ be a piecewise polynomial of degree $k \geq 0$ on each $T \in \mathcal{T}_{h}$, and let $\boldsymbol{\rho} \in \boldsymbol{L}^{2}(\Omega)$ be such that $\operatorname{curl}(\boldsymbol{\rho})=0$ in $\Omega$. Then, there exist $c, \widetilde{c}>0$, independent of $h$, such that

$$
\left\|\operatorname{curl}\left(\boldsymbol{\rho}_{h}\right)\right\|_{0, T} \leq c h_{T}^{-1}\left\|\boldsymbol{\rho}-\boldsymbol{\rho}_{h}\right\|_{0, T} \quad \forall T \in \mathcal{T}_{h}
$$

and

$$
\left\|\left[\boldsymbol{\rho}_{h} \boldsymbol{s}\right]\right\|_{0, e} \leq \widetilde{c} h_{e}^{-1 / 2}\left\|\boldsymbol{\rho}-\boldsymbol{\rho}_{h}\right\|_{0, \omega_{e}} \quad \forall e \in \mathcal{E}_{h}(\Omega) .
$$

Proof. For the proof of (5.35) we refer to [10, Lemma 4.3], whereas (5.36) is a slight modification of the proof of [10, Lemma 4.4]. We omit further details.

Furthermore, as announced in the statement of Theorem 5.1, throughout the rest of this section we assume for simplicity that $\boldsymbol{f}$ and $g$ are piecewise polynomials. The assumption on $\boldsymbol{f}$ is required by the next five lemmas in order to guarantee that $\mathbf{r}\left(\boldsymbol{u}_{h}, p_{h} ; \boldsymbol{f}\right)$ and all the other residual expressions involving this term become piecewise polynomials as well. In this way, Lemmas 5.9 and 5.10 can be applied to each one of them when deriving the corresponding efficiency estimates. Similarly, the assumption on $g$ insures that $\left(g-\boldsymbol{u}_{h} \cdot \boldsymbol{\nu}\right)$ shares the same property, which is needed below in the proof of our last lemma.

We now let $\mathbf{r}(\boldsymbol{u}, p ; \boldsymbol{f}):=\left(\gamma \boldsymbol{f}+\gamma p \boldsymbol{f}-\alpha_{0} \gamma \boldsymbol{u}\right)$ in $\Omega$, and notice from Theorem 3.2 that there holds $\mathbf{r}(\boldsymbol{u}, p ; \boldsymbol{f})=-\nabla p$ in $\Omega$. In addition, it is easy to see that

$$
\mathbf{r}(\boldsymbol{u}, p ; \boldsymbol{f})-\mathbf{r}\left(\boldsymbol{u}_{h}, p_{h} ; \boldsymbol{f}\right)=\gamma\left(p-p_{h}\right) \boldsymbol{f}-\alpha_{0} \gamma\left(\boldsymbol{u}-\boldsymbol{u}_{h}\right),
$$

and hence

$$
\left\|\mathbf{r}(\boldsymbol{u}, p ; \boldsymbol{f})-\mathbf{r}\left(\boldsymbol{u}_{h}, p_{h} ; \boldsymbol{f}\right)\right\|_{0, T} \leq \gamma\|\boldsymbol{f}\|_{\infty, \Omega}\left\|p-p_{h}\right\|_{0, T}+\alpha_{0} \gamma\left\|\boldsymbol{u}-\boldsymbol{u}_{h}\right\|_{0, T} \quad \forall T \in \mathcal{T}_{h}
$$

We continue our efficiency analysis with the following lemma.

Lemma 5.13. There exist $C_{1}, C_{2}>0$, independent of $h$, but depending on $\gamma, \alpha_{0}$, and $\|\boldsymbol{f}\|_{\infty, \Omega}$, such that

$$
h_{T}^{2}\left\|\operatorname{curl}\left\{\mathbf{r}\left(\boldsymbol{u}_{h}, p_{h} ; \boldsymbol{f}\right)\right\}\right\|_{0, T}^{2} \leq C_{1}\left\{\left\|p-p_{h}\right\|_{0, T}^{2}+\left\|\boldsymbol{u}-\boldsymbol{u}_{h}\right\|_{0, T}^{2}\right\} \quad \forall T \in \mathcal{T}_{h}
$$

and

$$
h_{e}\left\|\left[\mathbf{r}\left(\boldsymbol{u}_{h}, p_{h} ; \boldsymbol{f}\right) \cdot \boldsymbol{s}\right]\right\|_{0, e}^{2} \leq C_{2}\left\{\left\|p-p_{h}\right\|_{0, \omega_{e}}^{2}+\left\|\boldsymbol{u}-\boldsymbol{u}_{h}\right\|_{0, \omega_{e}}^{2}\right\} \quad \forall e \in \mathcal{E}_{h}(\Omega) .
$$

Proof. Since $\operatorname{curl}\{\mathbf{r}(\boldsymbol{u}, p ; \boldsymbol{f})\}=-\operatorname{curl} \nabla p=0$ in $\Omega$, it suffices to apply Lemma 5.12 to $\boldsymbol{\rho}=\mathbf{r}(\boldsymbol{u}, p ; \boldsymbol{f})$ and $\boldsymbol{\rho}_{h}=\mathbf{r}\left(\boldsymbol{u}_{h}, p_{h} ; \boldsymbol{f}\right)$, and then employ the estimate (5.37).

The efficiency estimate for the remaining residual term on each $T \in \mathcal{T}_{h}$ is given next.

Lemma 5.14. There exists $C_{3}>0$, independent of $h$, but depending on $\gamma, \alpha_{0}$, and $\|\boldsymbol{f}\|_{\infty, \Omega}$, such that

$$
h_{T}^{2}\left\|\mathbf{r}\left(\boldsymbol{u}_{h}, p_{h} ; \boldsymbol{f}\right)\right\|_{0, T}^{2} \leq C\left\{\left\|p-p_{h}\right\|_{0, T}^{2}+h_{T}^{2}\left\|\boldsymbol{u}-\boldsymbol{u}_{h}\right\|_{0, T}^{2}\right\} \quad \forall T \in \mathcal{T}_{h}
$$


Proof. It is similar to the proof of [28, Lemma 20]. Given $T \in \mathcal{T}_{h}$ we denote $\gamma_{T}:=\mathbf{r}\left(\boldsymbol{u}_{h}, p_{h} ; \boldsymbol{f}\right)$ in $T$. Applying (5.30) to $\boldsymbol{\gamma}_{T}$ and then subtracting and adding $\mathbf{r}(\boldsymbol{u}, p ; \boldsymbol{f})$, we find that

$$
\begin{gathered}
\left\|\boldsymbol{\gamma}_{T}\right\|_{0, T}^{2} \leq c_{1}\left\|\psi_{T}^{1 / 2} \gamma_{T}\right\|_{0, T}^{2}=c_{1} \int_{T} \psi_{T} \boldsymbol{\gamma}_{T} \cdot \mathbf{r}\left(\boldsymbol{u}_{h}, p_{h} ; \boldsymbol{f}\right) \\
=\quad c_{1} \int_{T} \psi_{T} \boldsymbol{\gamma}_{T} \cdot\left\{\mathbf{r}\left(\boldsymbol{u}_{h}, p_{h} ; \boldsymbol{f}\right)-\mathbf{r}(\boldsymbol{u}, p ; \boldsymbol{f})\right\}-c_{1} \int_{T} \psi_{T} \boldsymbol{\gamma}_{T} \cdot \nabla p .
\end{gathered}
$$

Next, taking into account that under the present choice of the space for $p_{h}$ (cf. (4.10) with $k=0$ ) there holds $\nabla p_{h}=0$ in each $T \in \mathcal{T}_{h}$, and integrating by parts, we obtain

$$
\int_{T} \psi_{T} \gamma_{T} \cdot \nabla p=\int_{T} \psi_{T} \gamma_{T} \cdot \nabla\left(p-p_{h}\right)=-\int_{T}\left(p-p_{h}\right) \operatorname{div}\left(\psi_{T} \gamma_{T}\right),
$$

which replaced back into (5.40) leads to

$$
\left\|\boldsymbol{\gamma}_{T}\right\|_{0, T}^{2} \leq c_{1} \int_{T} \psi_{T} \boldsymbol{\gamma}_{T} \cdot\left\{\mathbf{r}\left(\boldsymbol{u}_{h}, p_{h} ; \boldsymbol{f}\right)-\mathbf{r}(\boldsymbol{u}, p ; \boldsymbol{f})\right\}+c_{1} \int_{T}\left(p-p_{h}\right) \operatorname{div}\left(\psi_{T} \boldsymbol{\gamma}_{T}\right) .
$$

Hence, applying the Cauchy-Schwarz inequality and the inverse estimate (5.33), and performing some minor simplifications, we deduce from (5.41) that

$$
\begin{aligned}
\left\|\gamma_{T}\right\|_{0, T}^{2} & \leq c_{1}\left\|\psi_{T} \gamma_{T}\right\|_{0, T}\left\|\mathbf{r}\left(\boldsymbol{u}_{h}, p_{h} ; \boldsymbol{f}\right)-\mathbf{r}(\boldsymbol{u}, p ; \boldsymbol{f})\right\|_{0, T}+c_{1}\left\|p-p_{h}\right\|_{0, T}\left|\psi_{T} \gamma_{T}\right|_{1, T} \\
& \leq C\left\{\left\|\mathbf{r}\left(\boldsymbol{u}_{h}, p_{h} ; \boldsymbol{f}\right)-\mathbf{r}(\boldsymbol{u}, p ; \boldsymbol{f})\right\|_{0, T}+h_{T}^{-1}\left\|p-p_{h}\right\|_{0, T}\right\}\left\|\gamma_{T}\right\|_{0, T},
\end{aligned}
$$

which yields

$$
h_{T}\left\|\boldsymbol{\gamma}_{T}\right\|_{0, T} \leq C\left\{\left\|p-p_{h}\right\|_{0, T}+h_{T}\left\|\mathbf{r}\left(\boldsymbol{u}_{h}, p_{h} ; \boldsymbol{f}\right)-\mathbf{r}(\boldsymbol{u}, p ; \boldsymbol{f})\right\|_{0, T}\right\} .
$$

Finally, (5.37) and (5.42) imply the required inequality (5.39) and finish the proof.

We now estimate the residual term involving the tangential derivative of $\lambda_{h}$.

Lemma 5.15. There exists $C_{4}>0$, independent of $h$, but depending on $\gamma, \alpha_{0}$, and $\|\boldsymbol{f}\|_{\infty, \Omega}$, such that

$$
\begin{aligned}
& \sum_{e \in \mathcal{E}_{h}\left(\Gamma_{N}\right)} h_{e}\left\|\mathbf{r}\left(\boldsymbol{u}_{h}, p_{h} ; \boldsymbol{f}\right) \cdot \boldsymbol{s}-\frac{d \lambda_{h}}{d \boldsymbol{s}}\right\|_{0, e}^{2} \\
& \leq C_{4}\left\{\sum_{e \in \mathcal{E}_{h}\left(\Gamma_{N}\right)}\left\{\left\|\boldsymbol{u}-\boldsymbol{u}_{h}\right\|_{0, T_{e}}^{2}+\left\|p-p_{h}\right\|_{0, T_{e}}^{2}\right\}+\left\|\lambda_{h}-\lambda\right\|_{0 ; 1 / 2, \Gamma_{N}}^{2}\right\},
\end{aligned}
$$

where, given $e \in \mathcal{E}_{h}\left(\Gamma_{N}\right), T_{e}$ is the triangle of $\mathcal{T}_{h}$ having e as an edge.

Proof. We adapt the proof of [28, Lemma 22]. In fact, given $e \in \mathcal{E}_{h}\left(\Gamma_{N}\right)$ we first let

$$
\gamma_{e}:=\mathbf{r}\left(\boldsymbol{u}_{h}, p_{h} ; \boldsymbol{f}\right) \cdot \boldsymbol{s}-\frac{d \lambda_{h}}{d \boldsymbol{s}} \quad \text { on } \quad e .
$$

Then, thanks to (5.31) and the extension operator $L: C(e) \rightarrow C(T)$, we obtain that

$$
\begin{aligned}
\left\|\gamma_{e}\right\|_{0, e}^{2} \leq c_{2} \int_{e} \psi_{e} \gamma_{e}\left\{\mathbf{r}\left(\boldsymbol{u}_{h}, p_{h} ; \boldsymbol{f}\right) \cdot \boldsymbol{s}-\frac{d \lambda_{h}}{d \boldsymbol{s}}\right\} \\
\quad=c_{2} \int_{\partial T_{e}} \psi_{e} L\left(\boldsymbol{\gamma}_{e}\right)\left\{\mathbf{r}\left(\boldsymbol{u}_{h}, p_{h} ; \boldsymbol{f}\right) \cdot \boldsymbol{s}\right\}-c_{2} \int_{e} \psi_{e} \gamma_{e} \frac{d \lambda_{h}}{d \boldsymbol{s}} .
\end{aligned}
$$


Next, integrating by parts and then subtracting and adding $\mathbf{r}(\boldsymbol{u}, p ; \boldsymbol{f})=-\nabla p$ in $\Omega$, we find that

$$
\begin{aligned}
\int_{\partial T_{e}} & \psi_{e} L\left(\gamma_{e}\right)\left\{\mathbf{r}\left(\boldsymbol{u}_{h}, p_{h} ; \boldsymbol{f}\right) \cdot \boldsymbol{s}\right\} \\
& =-\int_{T_{e}} \operatorname{curl}\left(\psi_{e} L\left(\boldsymbol{\gamma}_{e}\right)\right) \cdot \mathbf{r}\left(\boldsymbol{u}_{h}, p_{h} ; \boldsymbol{f}\right)+\int_{T_{e}} \psi_{e} L\left(\boldsymbol{\gamma}_{e}\right) \operatorname{curl}\left\{\mathbf{r}\left(\boldsymbol{u}_{h}, p_{h} ; \boldsymbol{f}\right)\right\}
\end{aligned}
$$

and

$$
\begin{aligned}
\int_{T_{e}} \operatorname{curl}\left(\psi_{e} L\left(\boldsymbol{\gamma}_{e}\right)\right): \cdot \mathbf{r}\left(\boldsymbol{u}_{h}, p_{h} ; \boldsymbol{f}\right) \\
\quad=\int_{T_{e}} \operatorname{curl}\left(\psi_{e} L\left(\boldsymbol{\gamma}_{e}\right)\right) \cdot\left\{\mathbf{r}\left(\boldsymbol{u}_{h}, p_{h} ; \boldsymbol{f}\right)-\mathbf{r}(\boldsymbol{u}, p ; \boldsymbol{f})\right\}-\int_{T_{e}} \operatorname{curl}\left(\psi_{e} L\left(\boldsymbol{\gamma}_{e}\right)\right) \cdot \nabla p \\
=\int_{T_{e}} \operatorname{curl}\left(\psi_{e} L\left(\boldsymbol{\gamma}_{e}\right)\right) \cdot\left\{\mathbf{r}\left(\boldsymbol{u}_{h}, p_{h} ; \boldsymbol{f}\right)-\mathbf{r}(\boldsymbol{u}, p ; \boldsymbol{f})\right\}+\left\langle\frac{d p}{d s}, \psi_{e} L\left(\boldsymbol{\gamma}_{e}\right)\right\rangle_{\partial T_{e}},
\end{aligned}
$$

where $\langle\cdot, \cdot\rangle_{\partial T_{e}}$ denotes the duality pairing between $H^{-1 / 2}\left(\partial T_{e}\right)$ and $H^{1 / 2}\left(\partial T_{e}\right)$. In this way, using that $p=-\lambda$ in $\Gamma_{N}$ (cf. Theorem 3.2), we arrive at

$$
\begin{aligned}
\left\|\gamma_{e}\right\|_{0, e}^{2} \leq c_{2}\left\{-\int_{T_{e}} \operatorname{curl}\left(\psi_{e} L\left(\gamma_{e}\right)\right) \cdot\left\{\mathbf{r}\left(\boldsymbol{u}_{h}, p_{h} ; \boldsymbol{f}\right)-\mathbf{r}(\boldsymbol{u}, p ; \boldsymbol{f})\right\}\right. \\
\left.+\int_{T_{e}} \psi_{e} L\left(\gamma_{e}\right) \operatorname{curl}\left\{\mathbf{r}\left(\boldsymbol{u}_{h}, p_{h} ; \boldsymbol{f}\right)\right\}+\left\langle\frac{d}{d s}\left(\lambda-\lambda_{h}\right), \psi_{e} L\left(\gamma_{e}\right)\right\rangle_{e}\right\}
\end{aligned}
$$

where $\langle\cdot, \cdot\rangle_{e}$ denotes the duality pairing between $\left(H_{00}^{1 / 2}(e)\right)^{\prime}$ and $H_{00}^{1 / 2}(e)$. Here, as usual, $H_{00}^{1 / 2}(e)$ stands for the space of traces on $e$ of those elements in $H^{1}\left(T_{e}\right)$ whose traces vanish on $\partial T_{e} \backslash e$.

Now, since $\psi_{e} \gamma_{e} \in H_{00}^{1 / 2}(e)$ for each $e \in \mathcal{E}_{h}\left(\Gamma_{N}\right)$, we can write

$$
\sum_{e \in \mathcal{E}_{h}\left(\Gamma_{N}\right)} h_{e}\left\langle\frac{d}{d s}\left(\lambda-\lambda_{h}\right), \psi_{e} \gamma_{e}\right\rangle_{e}=\left\langle\frac{d}{d s}\left(\lambda-\lambda_{h}\right), \gamma\right\rangle_{\Gamma_{N}}
$$

where $\gamma$ is the piecewise polynomial defined by $\left.\gamma\right|_{e}:=h_{e} \psi_{e} \gamma_{e}$ on each $e \in \mathcal{E}_{h}\left(\Gamma_{N}\right)$. Then, applying the boundedness of the tangential derivative $\frac{d}{d s}: H_{00}^{1 / 2}\left(\Gamma_{N}\right) \rightarrow H_{00}^{-1 / 2}\left(\Gamma_{N}\right)$ (cf. [43]), employing the inverse estimate $\|\boldsymbol{\gamma}\|_{0 ; 1 / 2, \Gamma_{N}} \leq c h^{-1 / 2}\|\gamma\|_{0, \Gamma_{N}}$ (cf. [8, 46]), which certainly makes use of the quasi-uniformity of $\Gamma_{N, h}$, and using that $h_{e} \leq h$ and $0 \leq \psi_{e} \leq 1$, we deduce that

$$
\begin{gathered}
\sum_{e \in \mathcal{E}_{h}\left(\Gamma_{N}\right)} h_{e}\left\langle\frac{d}{d s}\left(\lambda-\lambda_{h}\right), \psi_{e} \gamma_{e}\right\rangle_{e} \leq C h^{-1 / 2}\left\|\lambda-\lambda_{h}\right\|_{0 ; 1 / 2, \Gamma_{N}}\|\gamma\|_{0, \Gamma_{N}} \\
\leq C\left\|\lambda-\lambda_{h}\right\|_{0 ; 1 / 2, \Gamma_{N}}\left\{\sum_{e \in \mathcal{E}_{h}\left(\Gamma_{N}\right)} h_{e}\left\|\gamma_{e}\right\|_{0, e}^{2}\right\}^{1 / 2} .
\end{gathered}
$$

On the other hand, applying Cauchy-Schwarz inequality, the inverse estimate (5.33), the fact that $h_{e} \leq h_{T}$, the inequality

$$
\left\|\psi_{e} L\left(\gamma_{e}\right)\right\|_{0, T_{e}} \leq c h_{e}^{1 / 2}\left\|\gamma_{e}\right\|_{0, e},
$$

which follows from (5.32), and the upper bound for $h_{T_{e}}^{2}\left\|\operatorname{curl}\left\{\mathbf{r}\left(\boldsymbol{u}_{h}, p_{h} ; \boldsymbol{f}\right)\right\}\right\|_{0, T_{e}}^{2}$ (cf. Lemma 5.13), we 
are able to show that

$$
\begin{aligned}
\sum_{e \in \mathcal{E}_{h}\left(\Gamma_{N}\right)} h_{e} & \left\{-\int_{T_{e}} \operatorname{curl}\left(\psi_{e} L\left(\boldsymbol{\gamma}_{e}\right)\right) \cdot\left\{\mathbf{r}\left(\boldsymbol{u}_{h}, p_{h} ; \boldsymbol{f}\right)-\mathbf{r}(\boldsymbol{u}, p ; \boldsymbol{f})\right\}+\int_{T_{e}} \psi_{e} L\left(\boldsymbol{\gamma}_{e}\right) \operatorname{curl}\left\{\mathbf{r}\left(\boldsymbol{u}_{h}, p_{h} ; \boldsymbol{f}\right)\right\}\right\} \\
& \leq C\left\{\sum_{e \in \mathcal{E}_{h}\left(\Gamma_{N}\right)}\left\|\boldsymbol{u}-\boldsymbol{u}_{h}\right\|_{0, T_{e}}+\left\|p-p_{h}\right\|_{0, T_{e}}\right\}^{1 / 2}\left\{\sum_{e \in \mathcal{E}_{h}\left(\Gamma_{N}\right)} h_{e}\left\|\boldsymbol{\gamma}_{e}\right\|_{0, e}^{2}\right\}^{1 / 2}
\end{aligned}
$$

Finally, inequalities (5.44), (5.45) and (5.46) lead to (5.43), thus completing the proof.

We remark that the estimate provided by the previous lemma is going to be the only nonlocal bound of the present efficiency analysis. Moreover, it is the only one needing to assume the quasi-uniformity of $\Gamma_{N, h}$. However, in the following lemma we show that, under an additional regularity assumption on $\lambda$, but without requiring any quasi-uniformity condition, a local estimate can be derived.

Lemma 5.16. Assume that $\left.\lambda\right|_{e} \in H^{1}(e)$ for each $e \in \mathcal{E}_{h}\left(\Gamma_{N}\right)$. Then there exists $C_{5}>0$, independent of $h$, but depending on $\gamma, \alpha_{0}$, and $\|\boldsymbol{f}\|_{\infty, \Omega}$, such that for each $e \in \mathcal{E}_{h}\left(\Gamma_{N}\right)$ there holds

$$
h_{e}\left\|\mathbf{r}\left(\boldsymbol{u}_{h}, p_{h} ; \boldsymbol{f}\right) \cdot \boldsymbol{s}-\frac{d \lambda_{h}}{d \boldsymbol{s}}\right\|_{0, e}^{2} \leq C\left\{\left\|\boldsymbol{u}-\boldsymbol{u}_{h}\right\|_{0, T_{e}}^{2}+\left\|p-p_{h}\right\|_{0, T_{e}}^{2}+h_{e}\left\|\frac{d}{d \boldsymbol{s}}\left(\lambda-\lambda_{h}\right)\right\|_{0, e}^{2}\right\},
$$

where $T_{e}$ is the triangle of $\mathcal{T}_{h}$ having e as an edge.

Proof. Similarly as in the proof of [28, Lemma 23], it suffices to reconsider the local estimate (5.44), and observe that, as a consequence of the Cauchy-Schwarz inequality, the last term of it is bounded by $\left\|\frac{d}{d \boldsymbol{s}}\left(\lambda-\lambda_{s}\right)\right\|_{0, e}\|\boldsymbol{\gamma}\|_{0, e}$. The rest follows exactly as in the last part of the proof of Lemma 5.15. We omit further details.

We now deal with the last term involving the residual $\mathbf{r}\left(\boldsymbol{u}_{h}, p_{h} ; \boldsymbol{f}\right)$.

Lemma 5.17. There exists $C_{6}>0$, independent of $h$, but depending on $\gamma, \alpha_{0}$, and $\|\boldsymbol{f}\|_{\infty, \Omega}$, such that for each $e \in \mathcal{E}_{h}\left(\Gamma_{D}\right)$ there holds

$$
h_{e}\left\|\mathbf{r}\left(\boldsymbol{u}_{h}, p_{h} ; \boldsymbol{f}\right) \cdot \boldsymbol{s}\right\|_{0, e}^{2} \leq C_{6}\left\{\left\|\boldsymbol{u}-\boldsymbol{u}_{h}\right\|_{0, T_{e}}^{2}+\left\|p-p_{h}\right\|_{0, T_{e}}^{2}\right\}
$$

where $T_{e}$ is the triangle of $\mathcal{T}_{h}$ having e as an edge.

Proof. We proceed as in the proof of [28, Lemma 21]. In fact, given $e \in \mathcal{E}_{h}\left(\Gamma_{D}\right)$ we denote $\gamma_{e}:=$ $\mathbf{r}\left(\boldsymbol{u}_{h}, p_{h} ; \boldsymbol{f}\right) \cdot \boldsymbol{s}$ on $e$. Since $p=0$ on $\Gamma_{D}($ cf. Theorem 3.2), we observe that $\mathbf{r}(\boldsymbol{u}, p ; \boldsymbol{f}) \cdot \boldsymbol{s}=(-\nabla p) \cdot \boldsymbol{s}=0$ on $\Gamma_{D}$, and hence $\mathbf{r}\left(\boldsymbol{u}_{h}, p_{h} ; \boldsymbol{f}\right) \cdot \boldsymbol{s}=\left\{\mathbf{r}\left(\boldsymbol{u}_{h}, p_{h} ; \boldsymbol{f}\right)-\mathbf{r}(\boldsymbol{u}, p ; \boldsymbol{f})\right\} \cdot \boldsymbol{s}$ on $e$. Then, applying (5.31) and the extension operator $L: C(e) \rightarrow C(T)$, we obtain that

$$
\begin{aligned}
& \left\|\gamma_{e}\right\|_{0, e}^{2} \leq c_{2}\left\|\psi_{e}^{1 / 2} \gamma_{e}\right\|_{0, e}^{2}=c_{2} \int_{e} \psi_{e} \gamma_{e}\left\{\mathbf{r}\left(\boldsymbol{u}_{h}, p_{h} ; \boldsymbol{f}\right) \cdot \boldsymbol{s}\right\} \\
& =\quad c_{2} \int_{\partial T_{e}} \psi_{e} L\left(\boldsymbol{\gamma}_{e}\right)\left\{\left\{\mathbf{r}\left(\boldsymbol{u}_{h}, p_{h} ; \boldsymbol{f}\right)-\mathbf{r}(\boldsymbol{u}, p ; \boldsymbol{f})\right\} \cdot \boldsymbol{s}\right\} .
\end{aligned}
$$

Now, integrating by parts and using that $\operatorname{curl}\{\mathbf{r}(\boldsymbol{u}, p ; \boldsymbol{f})\}=\operatorname{curl}\{-\nabla p\}=0$ in $\Omega$, we find that

$$
\begin{aligned}
\int_{\partial T_{e}} \psi_{e} L\left(\gamma_{e}\right)\left\{\left\{\mathbf{r}\left(\boldsymbol{u}_{h}, p_{h} ; \boldsymbol{f}\right)-\mathbf{r}(\boldsymbol{u}, p ; \boldsymbol{f})\right\} \cdot s\right\} \\
\quad=\quad-\int_{T_{e}} \operatorname{curl}\left(\psi_{e} L\left(\gamma_{e}\right)\right) \cdot\left\{\mathbf{r}\left(\boldsymbol{u}_{h}, p_{h} ; \boldsymbol{f}\right)-\mathbf{r}(\boldsymbol{u}, p ; \boldsymbol{f})\right\}+\int_{T_{e}} \psi_{e} L\left(\gamma_{e}\right) \operatorname{curl}\left\{\mathbf{r}\left(\boldsymbol{u}_{h}, p_{h} ; \boldsymbol{f}\right)\right\} .
\end{aligned}
$$


In turn, thanks to the fact that $0 \leq \psi_{e} \leq 1$ and (5.32), we have that

$$
\left\|\psi_{e} L\left(\gamma_{e}\right)\right\|_{0, T_{e}} \leq\left\|\psi_{e}^{1 / 2} L\left(\gamma_{e}\right)\right\|_{0, T_{e}} \leq c h_{e}^{1 / 2}\left\|\gamma_{e}\right\|_{0, e} .
$$

Hence, applying the Cauchy-Schwarz inequality and the inverse estimate (5.33), we deduce from (5.48), (5.49), and (5.50) that

$$
\left\|\boldsymbol{\gamma}_{e}\right\|_{0, e}^{2} \leq C\left\{h_{T_{e}}^{-1}\left\{\left\|\boldsymbol{u}-\boldsymbol{u}_{h}\right\|_{0, T_{e}}+\left\|p-p_{h}\right\|_{0, T_{e}}\right\}+\left\|\operatorname{curl}\left\{\mathbf{r}\left(\boldsymbol{u}_{h}, p_{h} ; \boldsymbol{f}\right)\right\}\right\|_{0, T_{e}}\right\} h_{e}^{1 / 2}\left\|\boldsymbol{\gamma}_{e}\right\|_{0, e},
$$

which, using that $h_{e} \leq h_{T_{e}}$, yields

$$
h_{e}\left\|\gamma_{e}\right\|_{0, e}^{2} \leq C\left\{\left\|\boldsymbol{u}-\boldsymbol{u}_{h}\right\|_{0, T_{e}}^{2}+\left\|p-p_{h}\right\|_{0, T_{e}}^{2}+h_{T_{e}}^{2}\left\|\operatorname{curl}\left\{\mathbf{r}\left(\boldsymbol{u}_{h}, p_{h} ; \boldsymbol{f}\right)\right\}\right\|_{0, T_{e}}^{2}\right\} .
$$

This inequality and the upper bound for $h_{T_{e}}^{2}\left\|\operatorname{curl}\left\{\mathbf{r}\left(\boldsymbol{u}_{h}, p_{h} ; \boldsymbol{f}\right)\right\}\right\|_{0, T_{e}}^{2}$ (cf. (5.38)) imply (5.47) and complete the proof.

We end our analysis with the upper bounds for the terms involving the trace of $p$ and the Neumann boundary condition on $\Gamma_{N}$.

Lemma 5.18. There exists $C_{7}>0$, independent of $h$, but depending on $\gamma, \alpha_{0}$, and $\|\boldsymbol{f}\|_{\infty, \Omega}$, such that for each $e \in \mathcal{E}_{h}\left(\Gamma_{N}\right)$ there holds

$$
h_{e}\left\|\lambda_{h}+p_{h}\right\|_{0, e}^{2} \leq C_{7}\left\{\left\|p-p_{h}\right\|_{0, T_{e}}^{2}+h_{e}\left\|\lambda-\lambda_{h}\right\|_{0, e}^{2}+h_{T_{e}}^{2}\left\|\boldsymbol{u}-\boldsymbol{u}_{h}\right\|_{0, T_{e}}^{2}\right\}
$$

where $T_{e}$ is the triangle having e as an edge.

Proof. It is an adaptation of the proof of [28, Lemma 24]. Indeed, applying the triangle inequality, the fact that $\lambda=-p$ on $\Gamma_{N}$ (cf. Theorem 3.2), and the discrete trace inequality (5.34), we easily obtain that for each $e \in \mathcal{E}_{h}\left(\Gamma_{N}\right)$ there holds

$$
\begin{aligned}
& h_{e}\left\|\lambda_{h}+p_{h}\right\|_{0, e}^{2} \leq 2\left\{h_{e}\left\|\lambda-\lambda_{h}\right\|_{0, e}^{2}+h_{e}\left\|p_{h}-p\right\|_{0, e}^{2}\right\} \\
\leq & C\left\{h_{e}\left\|\lambda-\lambda_{h}\right\|_{0, e}^{2}+\left\|p-p_{h}\right\|_{0, T_{e}}^{2}+h_{T_{e}}^{2}\left|p-p_{h}\right|_{1, T_{e}}^{2}\right\} .
\end{aligned}
$$

Now, bearing in mind that $\nabla p_{h}=0$ on each $T \in \mathcal{T}_{h}$, recalling that $\nabla p=\mathbf{r}(\boldsymbol{u}, p ; \boldsymbol{f})$, and then subtracting and adding $\mathbf{r}\left(\boldsymbol{u}_{h}, p_{h} ; \boldsymbol{f}\right)$, we deduce that

$$
\begin{aligned}
& h_{T_{e}}^{2}\left|p-p_{h}\right|_{1, T_{e}}^{2}=h_{T_{e}}^{2}\|\nabla p\|_{0, T_{e}}^{2} \\
& \quad \leq 2 h_{T_{e}}^{2}\left\|\mathbf{r}(\boldsymbol{u}, p ; \boldsymbol{f})-\mathbf{r}\left(\boldsymbol{u}_{h}, p_{h} ; \boldsymbol{f}\right)\right\|_{0, T_{e}}^{2}+2 h_{T_{e}}^{2}\left\|\mathbf{r}\left(\boldsymbol{u}_{h}, p_{h} ; \boldsymbol{f}\right)\right\|_{0, T_{e}}^{2} .
\end{aligned}
$$

Hence, employing (5.37) and the upper bound for $h_{T_{e}}^{2}\left\|\mathbf{r}\left(\boldsymbol{u}_{h}, p_{h} ; \boldsymbol{f}\right)\right\|_{0, T_{e}}^{2}$ given by Lemma 5.14, we conclude from (5.52) and (5.53) the required inequality (5.51), which finishes the proof.

Lemma 5.19. There exists $C_{8}>0$, independent of $h$, such that for each $e \in \mathcal{E}_{h}\left(\Gamma_{N}\right)$ there holds

$$
h_{e}\left\|g-\boldsymbol{u}_{h} \cdot \boldsymbol{\nu}\right\|_{0, e}^{2} \leq C_{8}\left\{\left\|\boldsymbol{u}-\boldsymbol{u}_{h}\right\|_{0, T_{e}}^{2}+h_{T_{e}}^{2}\left\|\operatorname{div}\left(\boldsymbol{u}-\boldsymbol{u}_{h}\right)\right\|_{0, T_{e}}^{2}\right\}
$$

where $T_{e}$ is the triangle of $\mathcal{T}_{h}$ having $e$ as an edge. 
Proof. We adapt the proof of [28, Lemma 25]. In fact, given $e \in \mathcal{E}_{h}\left(\Gamma_{N}\right)$, we let $\gamma_{e}:=g-\boldsymbol{u}_{h} \cdot \boldsymbol{\nu}$ on $e$. Then, employing (5.31), the fact that $\boldsymbol{u} \cdot \boldsymbol{\nu}=g$ on $\Gamma_{N}$ (cf. Theorem 3.2), and the extension operator $L: C(e) \rightarrow C(T)$, and then integrating by parts in $T_{e}$, we deduce that

$$
\begin{gathered}
\left\|\gamma_{e}\right\|_{0, e}^{2} \leq c_{2}\left\|\psi_{e}^{1 / 2} \gamma_{e}\right\|_{0, e}^{2}=c_{2} \int_{e} \psi_{e} \gamma_{e}\left(\boldsymbol{u}-\boldsymbol{u}_{h}\right) \cdot \boldsymbol{\nu}=c_{2} \int_{\partial T_{e}} \psi_{e} L\left(\gamma_{e}\right)\left(\boldsymbol{u}-\boldsymbol{u}_{h}\right) \cdot \boldsymbol{\nu} \\
=c_{2} \int_{T_{e}}\left\{\nabla\left(\psi_{e} L\left(\gamma_{e}\right)\right) \cdot\left(\boldsymbol{u}-\boldsymbol{u}_{h}\right)+\psi_{e} L\left(\boldsymbol{\gamma}_{e}\right) \operatorname{div}\left(\boldsymbol{u}-\boldsymbol{u}_{h}\right)\right\} .
\end{gathered}
$$

Next, applying Cauchy-Schwarz inequality, the fact that $0 \leq \psi_{e} \leq 1$, the relation (5.32) and the inverse estimate (5.33), we find that

$$
\begin{gathered}
\left\|\boldsymbol{\gamma}_{e}\right\|_{0, e}^{2} \leq C\left\{h_{T_{e}}^{-1}\left\|\boldsymbol{u}-\boldsymbol{u}_{h}\right\|_{0, T_{e}}+\left\|\operatorname{div}\left(\boldsymbol{u}-\boldsymbol{u}_{h}\right)\right\|_{0, T_{e}}\right\}\left\|\psi_{e} L\left(\boldsymbol{\gamma}_{e}\right)\right\|_{0, T_{e}} \\
\leq C h_{e}^{1 / 2}\left\{h_{T_{e}}^{-1}\left\|\boldsymbol{u}-\boldsymbol{u}_{h}\right\|_{0, T_{e}}+\left\|\operatorname{div}\left(\boldsymbol{u}-\boldsymbol{u}_{h}\right)\right\|_{0, T_{e}}\right\}\left\|\gamma_{e}\right\|_{0, e},
\end{gathered}
$$

which, using that $h_{e} \leq h_{T_{e}}$, leads to

$$
h_{e}\left\|\gamma_{e}\right\|_{0, e}^{2} \leq C\left\{\left\|\boldsymbol{u}-\boldsymbol{u}_{h}\right\|_{0, T_{e}}^{2}+h_{T_{e}}^{2}\left\|\operatorname{div}\left(\boldsymbol{u}-\boldsymbol{u}_{h}\right)\right\|_{0, T_{e}}^{2}\right\} .
$$

thus providing (5.54) and finishing the proof.

We end this section by remarking that the efficiency of $\boldsymbol{\theta}$ follows straightforwardly from the estimate (5.29) and Lemmas 5.13 throughout 5.19.

\section{$6 \quad$ Numerical results}

In this section we present several numerical examples showing the performance of the mixed finite element scheme (4.1), confirming the reliability and efficiency of the a posteriori error estimator $\boldsymbol{\theta}$ derived in Section 5, and illustrating the behavior of the associated adaptive algorithm. We consider the specific finite element subspaces defined in Section 4.2 with $k=0$. More precisely, we take (4.9), (4.10), and (4.13) in $\mathbb{R}^{2}$, whereas (4.9), (4.10), and (4.11) are employed in $\mathbb{R}^{3}$. We begin by introducing additional notations. The variable $N$ stands for the number of degrees of freedom defining the finite element subspaces $\boldsymbol{H}_{h}$ and $\boldsymbol{Q}_{h}$ (equivalently, the number of unknowns of (4.1)), and the individual and global errors are denoted by:

$$
\begin{gathered}
\mathrm{e}(\boldsymbol{u}):=\left\|\boldsymbol{u}-\boldsymbol{u}_{h}\right\|_{\operatorname{div}, \Omega}, \quad \mathrm{e}(p):=\left\|p-p_{h}\right\|_{0, \Omega}, \quad \mathrm{e}(\lambda):=\left\|\lambda-\lambda_{h}\right\|_{0 ; 1 / 2, \Gamma_{N}}, \\
\text { and } \quad \mathrm{e}:=\left\{[\mathrm{e}(\boldsymbol{u})]^{2}+[\mathrm{e}(p)]^{2}+[\mathrm{e}(\lambda)]^{2}\right\}^{1 / 2}
\end{gathered}
$$

where $(\boldsymbol{u},(p, \lambda)) \in \boldsymbol{H} \times \boldsymbol{Q}$ and $\left(\boldsymbol{u}_{h},\left(p_{h}, \lambda_{h}\right)\right) \in \boldsymbol{H}_{h} \times \boldsymbol{Q}_{h}$ are the unique solutions of (3.1) and (4.1), respectively. Note that, according to the estimates for the interpolation of Sobolev spaces (cf. $[40$, Appendix B] $),\|\cdot\|_{0 ; 1 / 2, \Gamma_{N}}$ can be approximated by $|\cdot|_{1, \Gamma_{N}}^{1 / 2}\|\cdot\|_{0, \Gamma_{N}}^{1 / 2}$. Furthermore, we define the effectivity index

$$
\operatorname{eff}(\boldsymbol{\theta}):=\mathrm{e} / \boldsymbol{\theta}
$$

and we let $r(\boldsymbol{u}), r(p), r(\lambda)$, and $r$ be the experimental rates of convergence given by

$$
r(\boldsymbol{u}):=\frac{\log \left(\mathrm{e}(\boldsymbol{u}) / \mathrm{e}^{\prime}(\boldsymbol{u})\right)}{\log \left(h / h^{\prime}\right)}, r(p):=\frac{\log \left(\mathrm{e}(p) / \mathrm{e}^{\prime}(p)\right)}{\log \left(h / h^{\prime}\right)}, r(\lambda):=\frac{\log \left(\mathrm{e}(\lambda) / \mathrm{e}^{\prime}(\lambda)\right)}{\log \left(h / h^{\prime}\right)}, r:=\frac{\log \left(\mathrm{e} / \mathrm{e}^{\prime}\right)}{\log \left(h / h^{\prime}\right)},
$$


where $h$ and $h^{\prime}$ denote two consecutive meshsizes with errors e and $\mathrm{e}^{\prime}$, respectively. However, when Algorithm 1 is applied (see details below), the expression $\log \left(h / h^{\prime}\right)$ is replaced by $-\frac{1}{2} \log \left(N / N^{\prime}\right)$, where $N$ and $N^{\prime}$ denote the corresponding degrees of freedom of each triangulation. In addition, we denote the postprocessing error associated to the inverse change of variables needed to recover the original pressure field $P$ from $p_{h}$, and its associated rate as

$$
\mathrm{e}(P)=\left\|P+\gamma^{-1} \log \left(p_{h}+1\right)\right\|_{0, \Omega} \quad \text { and } \quad r(P):=\frac{\log \left(\mathrm{e}(P) / \mathrm{e}^{\prime}(P)\right)}{\log \left(h / h^{\prime}\right)} .
$$

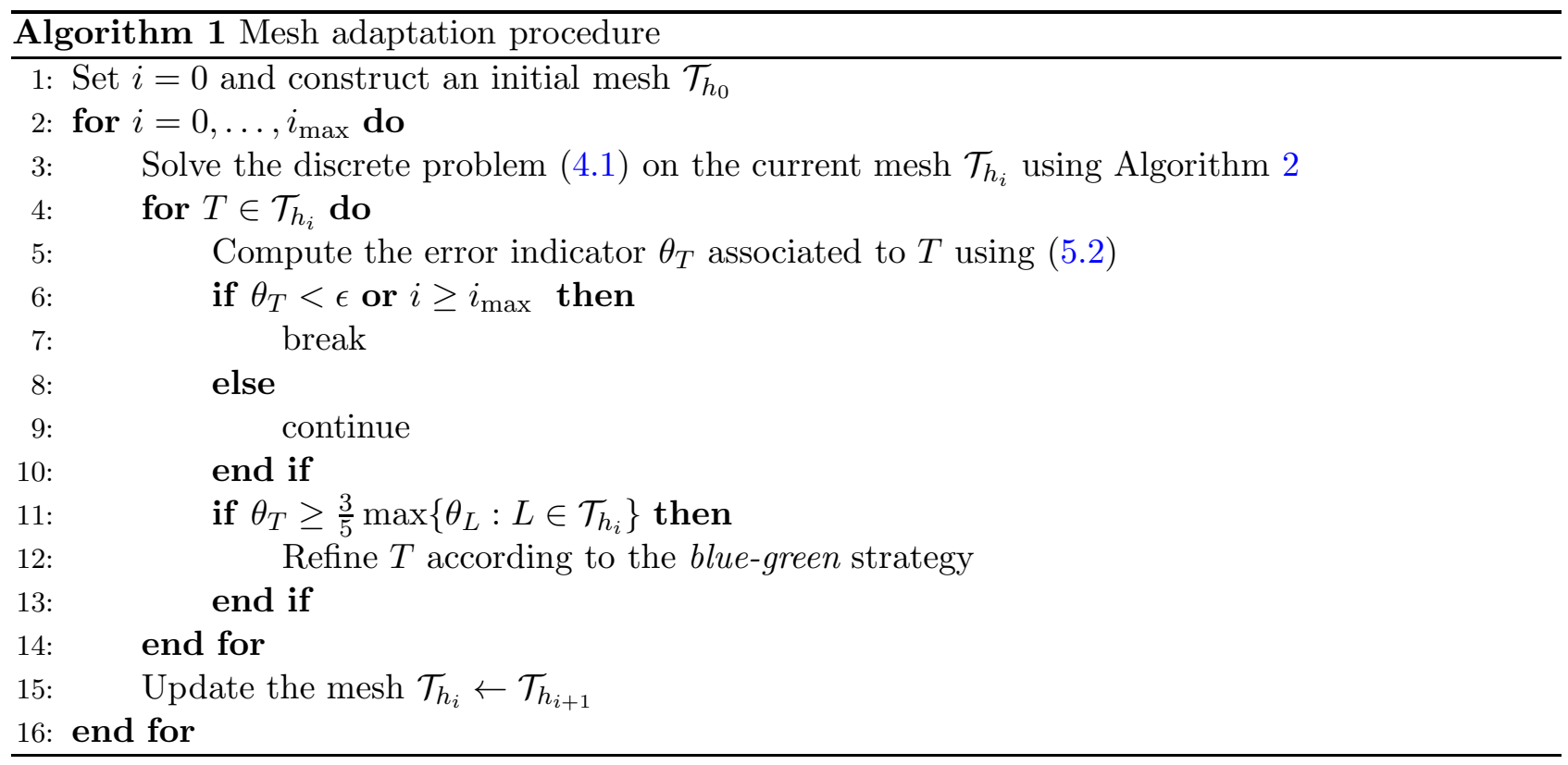

Following the analysis of the discrete problem, here a classical Picard (fixed point) algorithm is employed to treat (4.1), which ensures linear convergence [36]. The iterations are stopped when the $L^{2}$-norm of the pressure residual attains a chosen tolerance $\epsilon_{f p}$. The main steps are summarized in Algorithm 2.

In what follows we describe the examples to be considered, where the accuracy is assessed using the manufactured solution approach. In Example 1 we consider the domain $\Omega:=(0,1)^{2}$ with $\Gamma_{D}=$ $(0,1) \times\{0\}$ and $\Gamma_{N}=\partial \Omega \backslash \Gamma_{D}$, and choose $\boldsymbol{f}$ and $g$ so that the exact solutions of (2.1) and (2.2) are given by the smooth functions

$$
\begin{gathered}
U=\boldsymbol{u}\left(x_{1}, x_{2}\right):=\left(\begin{array}{c}
\sin \left(\pi x_{1}\right) \cos \left(\pi x_{2}\right), \\
-\cos \left(\pi x_{1}\right) \sin \left(\pi x_{2}\right)
\end{array}\right), \quad p\left(x_{1}, x_{2}\right):=x_{1}^{2}+x_{1} x_{2}, \\
\lambda\left(x_{1}, x_{2}\right):=-\left.p\right|_{\Gamma_{N}}, \quad P\left(x_{1}, x_{2}\right):=-\gamma^{-1} \log (p+1) .
\end{gathered}
$$

We set $\alpha_{0}=0.1, \gamma=10$ and study the accuracy of the discretization using piecewise constant approximations for the pressure field, $\mathbf{R T}_{0}$ approximations for velocities, and piecewise linear approximations for the Lagrange multiplier. Computed errors, convergence rates, effectivity indexes, and number of fixed point iterations to convergence are displayed in the top rows of Table 6.1. We observe optimal orders of convergence for all quantities and we notice that a fixed point tolerance of $\epsilon_{f p}=1 e-8$ is met, in average, at around twelve Picard iterations. On the bottom part of Table 6.1 we display the convergence history associated to an implementation of the discrete counterpart of the non-symmetric equivalent linear problem (3.3). For the former (and for all remaining examples) we employ a conjugate gradient solver, whereas for the latter we use the unsymmetric multi-frontal direct solver for 


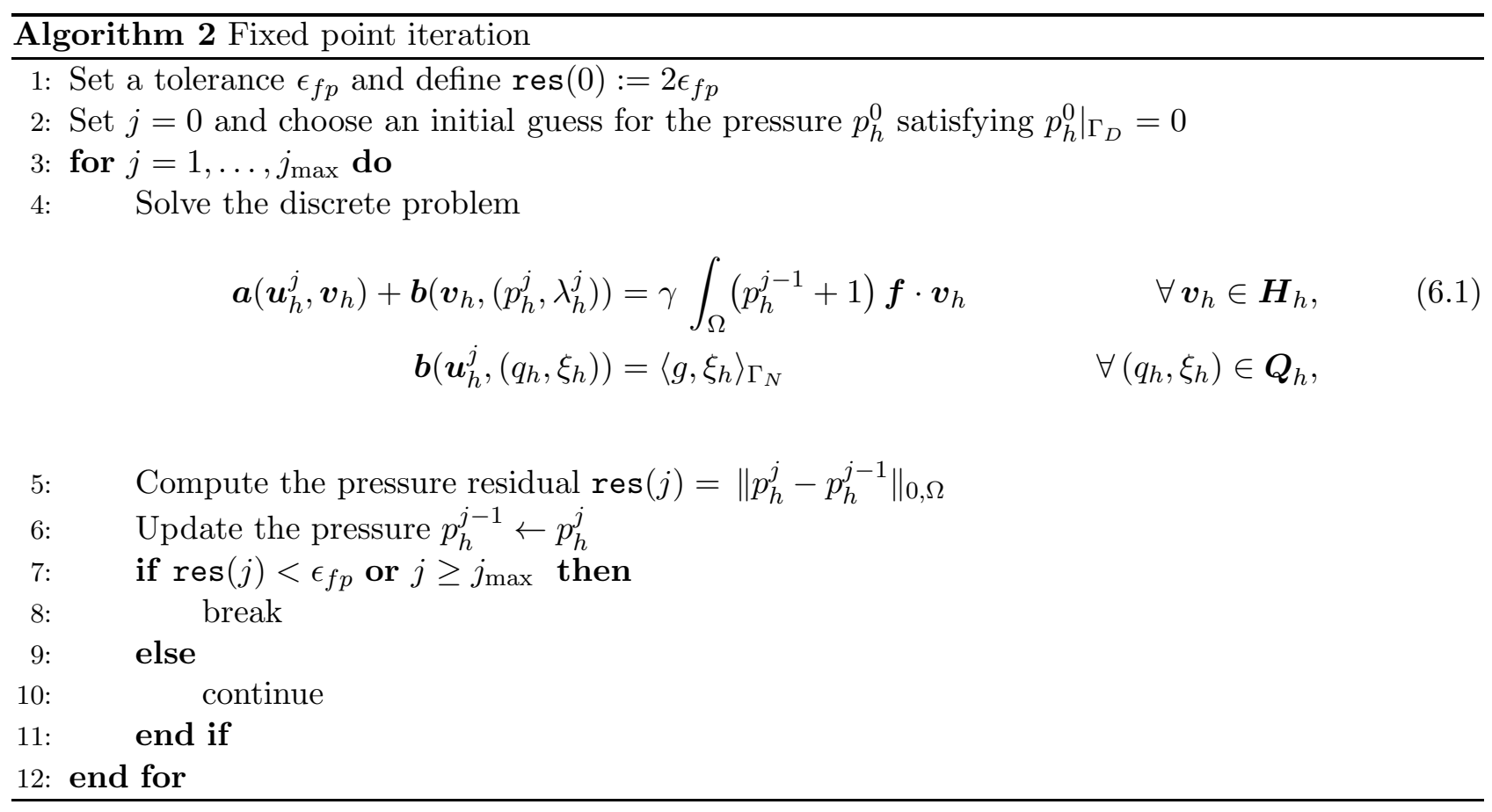

sparse matrices (UMFPACK). Since individual errors, effectivity indexes, and convergence rates are almost identical between the two methods, one could choose any of the two approaches. Here we focus on the first one, mainly since it corresponds to the scheme analyzed in the previous sections and its solution consists of solving symmetric systems. Nevertheless, the second one is certainly appealing from the computational viewpoint. For this example we display approximate velocity components, and original and modified pressures obtained on the finest level (using a mesh of 313041 vertices and 626080 elements) in Figure 6.1.

Next, Example 2 focuses on the nonconvex pacman domain $\Omega=\left\{\left(x_{1}, x_{2}\right) \in \mathbb{R}^{2}: x_{1}^{2}+x_{2}^{2} \leq\right.$ $1\} \backslash(0,1)^{2}$, with boundaries $\Gamma_{N}=(0,1) \times\{0\} \bigcup\{0\} \times(0,1)$ and $\Gamma_{D}=\partial \Omega \backslash \Gamma_{D}$, where the model problems (2.1),(2.2) admit the following exact solutions

$$
\begin{aligned}
& U= \boldsymbol{u}\left(x_{1}, x_{2}\right):=\left(\left(x_{1}-c\right)^{2}+\left(x_{2}-c\right)^{2}\right)^{-1 / 2}\left(\begin{array}{c}
c-x_{2} \\
x_{1}-c
\end{array}\right), p\left(x_{1}, x_{2}\right):=\frac{1-x_{1}^{2}-x_{2}^{2}}{\left(x_{1}-c\right)^{2}+\left(x_{2}-c\right)^{2}}, \\
& \lambda\left(x_{1}, x_{2}\right):=-\left.p\right|_{\Gamma_{N}}, \quad P\left(x_{1}, x_{2}\right):=-\gamma^{-1} \log (p+1),
\end{aligned}
$$

with $c=0.025$, satisfying the homogeneous boundary condition on $\Gamma_{D}$. Both pressure and velocity fields exhibit singularities close to the origin. This example is utilized to illustrate the behavior of the adaptive algorithm associated with $\boldsymbol{\theta}$, which is summarized in Algorithm 1 (see [54]). The corresponding error history for a quasi-uniform and an adaptive refinement strategy are reported in Table 6.2. In both cases we observe that, in comparison with Example 1, more fixed point iterations are needed to achieve the same tolerance. From the first part of the table, one also notices that the iteration count (based on a pressure residual) is largely affected by the presence of singularities. We also observe a hindered convergence, particularly of the Lagrange multiplier (in comparison with that of the previous example) as well as a much lower and oscillating effectivity index. These anomalies are amended by the adaptive strategy: In the bottom part of Table 6.2 we observe almost optimal convergence rates for all fields and, for the same fixed point tolerance, an average iteration count closer to the one reported in Table 6.1. This is also evidenced from Figure 6.2, where we plot the total error e versus the degrees of freedom for both refinement strategies and observe suboptimal convergence 


\begin{tabular}{|c|c|c|c|c|c|c|c|c|c|c|c|}
\hline$N$ & $h$ & $\mathrm{e}(\boldsymbol{u})$ & $r(\boldsymbol{u})$ & $\mathrm{e}(p)$ & $r(p)$ & $\mathrm{e}(\lambda)$ & $r(\lambda)$ & $\mathrm{e}(P)$ & $r(P)$ & $\operatorname{eff}(\boldsymbol{\theta})$ & iter \\
\hline \multicolumn{12}{|c|}{ Problem (3.1) solved iteratively via Algorithm 2} \\
\hline 11 & 1.414210 & 0.777153 & - & 0.454962 & - & 1.52585 & - & - & - & 0.238183 & 10 \\
\hline 32 & 0.707107 & 0.465914 & 0.539419 & 0.232287 & 0.969840 & 0.759940 & 0.956320 & 0.024021 & - & 0.264271 & 9 \\
\hline 104 & 0.353553 & 0.264449 & 0.817072 & 0.116629 & 0.993975 & 0.042501 & 2.247691 & 0.014861 & 0.692782 & 0.260534 & 10 \\
\hline 368 & 0.176777 & 0.137101 & 0.947764 & 40.058315 & 0.999992 & 0.015887 & 1.419630 & 0.007794 & 0.931046 & 0.253384 & 11 \\
\hline 1376 & 0.088388 & 0.069199 & 0.986393 & 0.029155 & 1.000112 & 0.008627 & 0.880807 & 0.003943 & 0.982878 & 0.250735 & 12 \\
\hline 5312 & 0.044194 & 0.034682 & 0.996562 & 0.014577 & 1.000041 & 0.004781 & 0.851562 & 0.001977 & 0.995583 & 0.249815 & 11 \\
\hline 20864 & 0.022097 & 0.017352 & 0.999138 & 0.007288 & 1.000011 & 0.002578 & 0.891039 & 0.000990 & 0.998306 & 0.249517 & 11 \\
\hline 82688 & 0.011049 & 0.008677 & 0.999784 & 40.003644 & 1.000010 & 0.001352 & 0.930602 & 0.000496 & 0.997220 & 0.249431 & 12 \\
\hline 329216 & 0.005524 & 0.004339 & 0.999946 & 0.001822 & 1.000000 & 0.000696 & 0.958398 & 0.000249 & 0.989965 & 0.249414 & 11 \\
\hline 1313792 & 0.002762 & 0.002169 & 0.999987 & 70.000911 & 1.000000 & 0.000354 & 0.975827 & 0.000128 & 0.961434 & 0.249416 & 11 \\
\hline \multicolumn{12}{|c|}{ Linear non-symmetric problem (3.3) } \\
\hline 11 & 1.414210 & 0.677153 & - & 0.454962 & - & 1.525851 & - & - & - & 0.238183 & 1 \\
\hline 32 & 0.707107 & 0.465914 & 0.539419 & 0.232287 & 0.969840 & 1.059940 & 0.525629 & 0.0240221 & - & 0.264271 & 1 \\
\hline 104 & 0.353553 & 0.264449 & 0.817072 & 20.116629 & 0.993975 & 0.042501 & 4.640351 & 0.0148613 & 0.692794 & 0.260534 & 1 \\
\hline 368 & 0.176777 & 0.137102 & 0.947764 & 40.058315 & 0.999992 & 0.015887 & 1.419632 & 0.007794 & 0.931050 & 0.253384 & 1 \\
\hline 1376 & 0.088388 & 0.069199 & 0.986393 & 0.029155 & 1.000112 & 0.008627 & 0.880807 & 0.0039436 & 0.982917 & 0.250732 & 1 \\
\hline 5312 & 0.044194 & 0.034682 & 0.996562 & 0.014577 & 1.000041 & 0.004781 & 0.851562 & 0.0019776 & 0.995739 & 0.249815 & 1 \\
\hline 20864 & 0.022097 & 0.017351 & 0.999138 & 0.007289 & 1.000010 & 0.002578 & 0.891039 & 0.0009895 & 0.998933 & 0.249517 & 1 \\
\hline 82688 & 0.011049 & 0.008677 & 0.999784 & 40.003644 & 1.000000 & 0.001352 & 0.930603 & 0.0004948 & 0.999721 & 0.249431 & 1 \\
\hline 329216 & 0.005524 & 0.004339 & 0.999946 & 0.001822 & 1.000000 & 0.000696 & 0.958398 & 0.0002474 & 0.999882 & 0.249414 & 1 \\
\hline 1313792 & 0.002762 & 0.002169 & 0.999987 & 70.000911 & 1.000000 & 0.000353 & 0.975827 & 0.000123 & 0.999771 & 0.249416 & 1 \\
\hline
\end{tabular}

Table 6.1: Example 1: Experimental convergence for the mixed finite element approximation of the Darcy problem (2.2) and postprocessed pressure $P_{h}=\gamma^{-1} \log \left(p_{h}+1\right)$ on a sequence of uniformly refined triangulations of $\Omega=(0,1)^{2}$, using a fixed point formulation with symmetric iterations (top) and a linear non-symmetric formulation (bottom). Here we have considered the parameters $\alpha_{0}=0.1$, $\gamma=10$.

(approximately of $O\left(h^{3 / 4}\right)$ ) for the quasi-uniform refinement. In addition, snapshots of the adapted meshes at different stages of the algorithm are displayed in Figure 6.3, exhibiting concentration of the adaptation procedure near the origin, which is the zone of highest gradients and which is well captured by the error estimator. We also show the approximate solutions on the finest level obtained with the same family of finite elements as in the previous example (see Figure 6.4).

Finally, in Example 3 we illustrate the applicability and accuracy of the proposed numerical method in a three-dimensional scenario. For this we consider $\Omega=(0,1)^{3}$ where the Dirichlet boundary is the bottom lid of the cube $\Gamma_{D}=(0,1) \times(0,1) \times\{0\}$, and the remaining faces constitute the Neumann boundary $\Gamma_{N}$. We construct $\boldsymbol{f}, g$ so that the exact solutions of the original and auxiliary Darcy problems (2.2), (2.2) are given by

$$
\begin{aligned}
& U=\boldsymbol{u}\left(x_{1}, x_{2}, x_{3}\right):=\left(\begin{array}{c}
\cos \left(2 \pi x_{1}\right) \sin \left(2 \pi x_{2}\right) \sin \left(2 \pi x_{3}\right) \\
\sin \left(2 \pi x_{1}\right) \cos \left(2 \pi x_{2}\right) \sin \left(2 \pi x_{3}\right) \\
-2 \sin \left(2 \pi x_{1}\right) \sin \left(2 \pi x_{2}\right) \cos \left(2 \pi x_{3}\right)
\end{array}\right) \\
& p\left(x_{1}, x_{2}, x_{3}\right):=\sin \left(2 \pi x_{1}\right) \sin \left(2 \pi x_{2}\right) \sin \left(2 \pi x_{3}\right)+x_{1} x_{2} x_{3}, \quad P\left(x_{1}, x_{2}, x_{3}\right):=-\gamma^{-1} \log (p+1), \\
& \lambda\left(x_{1}, x_{2}, x_{3}\right):=-\left.p\right|_{\Gamma_{N}} .
\end{aligned}
$$

As in the preceding tests, we choose the model parameters $\alpha_{0}=0.1, \gamma=10$. Using as a base an 

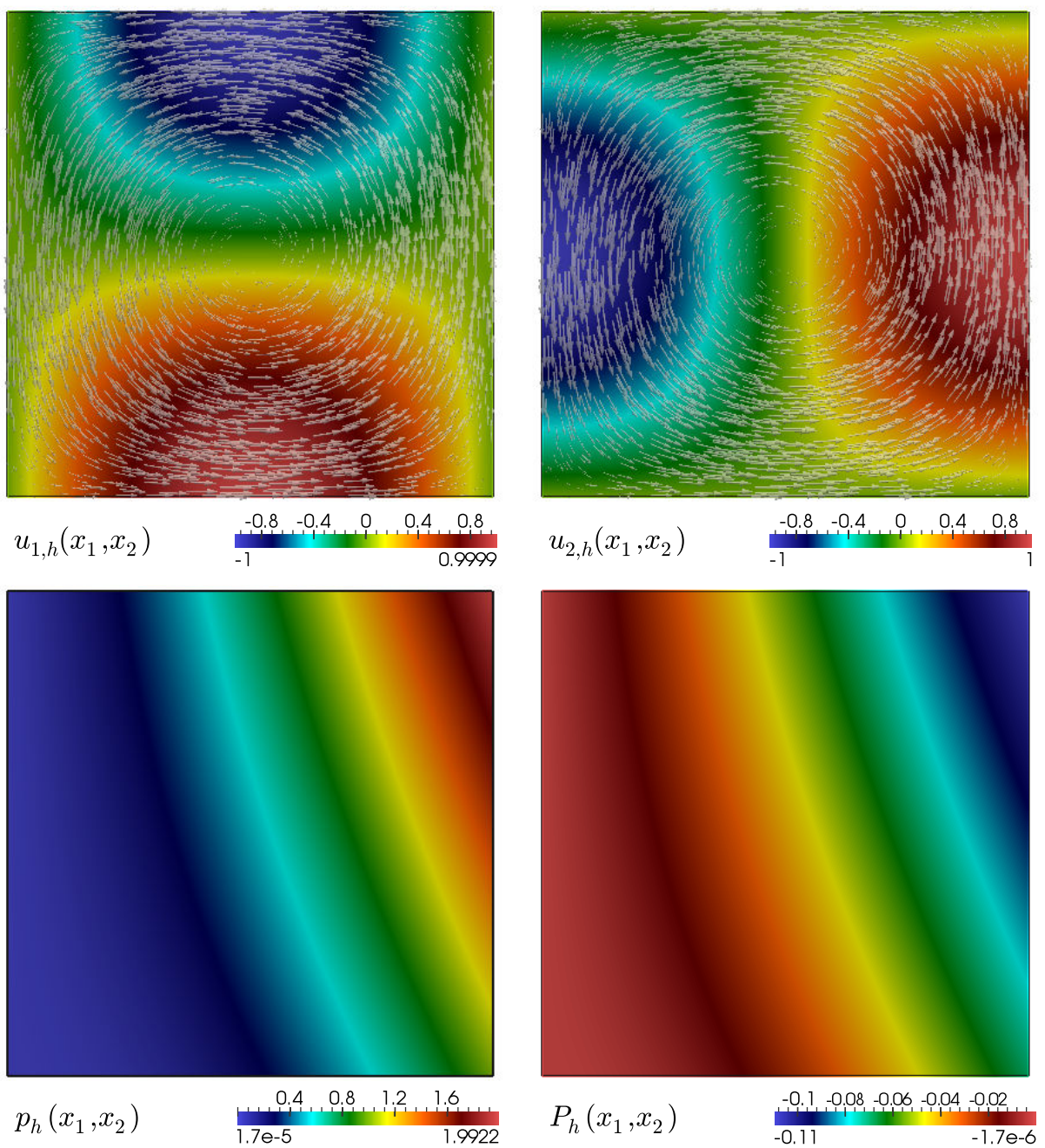

Figure 6.1: Example 1: Approximate velocity components (top), pressure distribution (bottom left), and postprocessed pressure (bottom right), computed using a mesh of 313041 vertices and 626080 elements.

initial tetrahedral mesh of 8 vertices and 18 elements, we perform eight successive refinements and we compute experimental errors in different norms. Now we relax the fixed point tolerance to $\epsilon_{f p}=1 e-6$ and observe that the average number of iterations to converge is 14 . Optimal rates of convergence are evidenced from Table 6.3. Approximate velocities and pressure obtained on the finest level (the corresponding mesh has 287496 vertices and 1698450 tetrahedral elements) are presented in Figure 6.5.

\section{References}

[1] Agmon, S., Lectures on Elliptic Boundary Value Problems. Van Nostrand, Princeton, New Jersey, 1965.

[2] Ahusborde, E., Azaïez, M., Ben Belgacem, F. and Bernardi, C., Automatic simplification of Darcy's equations with pressure dependent permeability. ESAIM: Mathematical Modelling and Numerical Analysis, 47(6) (2013) 1797-1820. 


\begin{tabular}{|c|c|c|c|c|c|c|c|c|c|c|c|}
\hline$N$ & $h$ & $\mathrm{e}(\boldsymbol{u})$ & $r(\boldsymbol{u})$ & $\mathrm{e}(p)$ & $r(p)$ & $\mathrm{e}(\lambda)$ & $r(\lambda)$ & $\mathrm{e}(P)$ & $r(P)$ & $\operatorname{eff}(\boldsymbol{\theta})$ & iter \\
\hline \multicolumn{12}{|c|}{ Quasi-uniform refinement } \\
\hline 225 & .396463 & 11.43581 & - & 39.30172 & - & 5.905905 & - & 0.093927 & - & 0.035166 & 33 \\
\hline 675 & .234657 & 10.09455 & 0.116705 & 28.75717 & 0.595641 & 3.798687 & 0.757472 & 0.069779 & 0.792872 & 0.018529 & 34 \\
\hline 1625 & .153371 & 8.885424 & 0.244053 & 25.28118 & 0.302922 & 2.147508 & 0.661754 & 0.041202 & 0.838860 & 0.016287 & 32 \\
\hline 3530 & .102059 & 6.453057 & 0.218888 & 18.87943 & 0.716865 & 1.4 & 0.529988 & 0.0 & 0.776 & 0.013298 & 30 \\
\hline 7960 & .068675 & 5.761 & 0.400416 & 13.89542 & 0.773727 & 0.9 & 0.873166 & 0.0 & 0.820906 & 0.026307 & 27 \\
\hline 19930 & .043191 & 3.869 & 0.519 & 9.7 & 0.78 & 0.5 & 0.81 & & 0.88 & 790 & 33 \\
\hline 56730 & .025888 & 3.05 & 0.7276 & 6.67 & 0.822 & 0.3 & 0.72 & & 0.82 & 904 & 35 \\
\hline 178440 & .014914 & 1.62 & 1.146 & 4.555427 & 0.84 & 0 & 0.8 & & 0.6 & 482 & 32 \\
\hline 618660 & .008210 & 1.06 & 0.698373 & 2.461297 & 0.796696 & 0.123352 & & & & & 34 \\
\hline \multicolumn{12}{|c|}{ Adaptive refinement } \\
\hline 861 & .210978 & 5.282240 & - & 38.54491 & - & 5.709012 & - & 0.070252 & - & 0.766485 & 13 \\
\hline 1247 & .163169 & 3.171521 & 1.073922 & 19.82960 & 1.136872 & 4.367726 & 0.630054 & 0.045942 & 0.962860 & 0.768496 & 14 \\
\hline 1931 & .135411 & 1.75 & 1.015801 & 9.571121 & 1.033150 & 1.35 & 0.899 & & 1.011 & 0.769635 & 15 \\
\hline 3401 & .118863 & 0.90 & 1.038221 & 4.444 & 1.210593 & 0.86 & 0.970 & & 0.955 & 505 & 13 \\
\hline 6843 & .096292 & 0.56 & 1.052795 & 2.193142 & 1.320374 & & 0.982651 & & 0.940 & 7345 & 14 \\
\hline 17350 & .078102 & 0.167351 & 1.092846 & 1.290872 & 1.139381 & $0.3 s$ & 0.885738 & 31 & 0.948 & 0.750103 & 16 \\
\hline 46492 & .062494 & 0.13 & 0.930274 & 0.7960 & 0.980 & & 0.954 & & & 237 & 13 \\
\hline 127844 & .051637 & 0.084252 & 0.950035 & 0.481753 & 0.993065 & 0.17 & 0.893301 & 0.0 & 0.9631 & 0.731722 & 14 \\
\hline 329880 & .042528 & 0.038337 & 0.989902 & 0.311 & 0.982892 & & 0.986044 & & & 7151 & 13 \\
\hline 783742 & .036014 & 0.010492 & 0.978930 & 0.134684 & 0.946813 & 0.101078 & 0.959217 & 0.000642 & 0.930247 & 0.756734 & 14 \\
\hline
\end{tabular}

Table 6.2: Example 2: Experimental convergence for the mixed finite element approximation of the Darcy problem (2.2) and postprocessed pressure $P_{h}=\gamma^{-1} \log \left(p_{h}+1\right)$ on a sequence of quasi-uniformly (top) and adaptively (bottom) refined meshes of $\Omega=\left\{\left(x_{1}, x_{2}\right) \in \mathbb{R}^{2}: x_{1}^{2}+x_{2}^{2} \leq 1\right\} \backslash(0,1)^{2}$. Here we have considered the parameters $\alpha_{0}=0.1, \gamma=10$.

[3] Ainsworth, M. And Oden, J.T., A posteriori error estimation in finite element analysis. Computer Methods in Applied Mechanics and Engineering, 142(1-2) (1997) 1-88.

[4] Alonso, A., Error estimators for a mixed method. Numerische Mathematik, 74(4) (1996) 385395.

[5] Arnold, D.N., An interior penalty finite element method with discontinuous elements. SIAM Journal on Numerical Analysis, 19(4) (1982) 742-760.

[6] Azaïez, M., Ben Belgacem, F., Bernardi, C. and Chorfi, N., Spectral discretization of Darcy's equations with pressure dependent porosity. Applied Mathematics and Computation, 217(5) (2010) 1838-1856.

[7] Azaïez, M., Ben Belgacem, F., Grundmann, M. and Khallouf, H., Staggered grids hybrid-dual spectral element method for second order elliptic problems, Application to high-order time splitting methods for Navier-Stokes equations. Computer Methods in Applied Mechanics and Engineering, 166(3-4) (1998) 183-199.

[8] BabušKa, I. And AzIz, A.K., Survey lectures on the mathematical foundations of the finite element method. In: Aziz, A.K. (ed.) The Mathematical Foundations of the Finite Element Method with Applications to Partial Differential Equations. Academic Press, New York (1972). 


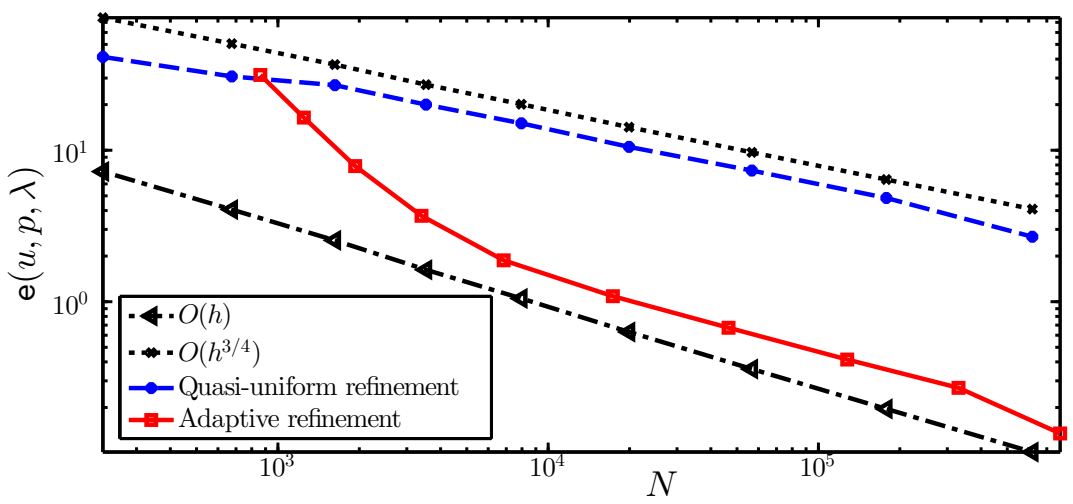

Figure 6.2: Example 2: Decay of the total error with respect to the number of degrees of freedom using a quasi-uniform and an adaptive refinement strategy (see individual errors in Table 6.2).

\begin{tabular}{rcccccccccc}
\hline$N$ & $h$ & $\mathrm{e}(\boldsymbol{u})$ & $r(\boldsymbol{u})$ & $\mathrm{e}(p)$ & $r(p)$ & $\mathrm{e}(\lambda)$ & $r(\lambda)$ & $\mathrm{e}(P)$ & $r(P)$ & iter \\
\hline \hline 32 & 1.414211 & 2.043353 & - & 0.495995 & - & 1.747510 & - & 0.124762 & - & 16 \\
195 & 0.707107 & 1.066271 & 0.968334 & 0.273696 & 0.857752 & 0.809612 & 1.152151 & 0.073987 & 0.794381 & 15 \\
2616 & 0.282843 & 0.519971 & 0.987055 & 0.155025 & 0.620363 & 0.426881 & 0.820118 & 0.029708 & 0.995827 & 15 \\
19931 & 0.141421 & 0.246593 & 1.076301 & 0.079143 & 0.969966 & 0.259207 & 0.879427 & 0.019232 & 0.627301 & 13 \\
96000 & 0.083189 & 0.145919 & 0.988807 & 0.046887 & 0.986583 & 0.168196 & 1.086805 & 0.011871 & 0.909225 & 12 \\
340107 & 0.054392 & 0.095618 & 0.994823 & 0.030734 & 0.994049 & 0.082207 & 1.063027 & 0.007858 & 0.971003 & 11 \\
974840 & 0.038222 & 0.067251 & 0.997451 & 0.021619 & 0.997142 & 0.041281 & 1.053195 & 0.005544 & 0.988516 & 10 \\
2397651 & 0.028284 & 0.034231 & 0.999417 & 0.015553 & 0.998497 & 0.021958 & 0.920208 & 0.003473 & 0.983695 & 14 \\
3256096 & 0.018757 & 0.018630 & 0.988616 & 0.007387 & 0.998462 & 0.011346 & 0.983085 & 0.001824 & 0.886841 & 14 \\
\hline
\end{tabular}

Table 6.3: Example 3: Convergence results for the mixed finite element approximation of the Darcy problem (2.2) and postprocessed pressure $P_{h}=\gamma^{-1} \log \left(p_{h}+1\right)$ on a sequence of uniformly refined triangulations of $\Omega=(0,1)^{3}$. Here we have considered the parameters $\alpha_{0}=0.1, \gamma=10$.

[9] BabušKa, I. And Gatica, G.N., On the mixed finite element method with Lagrange multipliers. Numerical Methods for Partial Differential Equations, 19(2) (2003) 192-210.

[10] Barrios, T.P., Gatica, G.N., González, M. and Heuer, N., A residual based a posteriori error estimator for an augmented mixed finite element method in linear elasticity. ESAIM: Mathematical Modelling and Numerical Analysis, 40(5) (2006) 843-869.

[11] Barus, C., Isotherms, isopiestics and isometrics relative to viscosity. American Journal of Science, 45 (1893) 87-96.

[12] Bernardi, C., Canuto, C. and Maday, Y., Generalized inf-sup conditions for Chebyshev spectral approximation of the Stokes problem. SIAM Journal on Numerical Analysis, 25(6) (1998) $1237-1271$. 

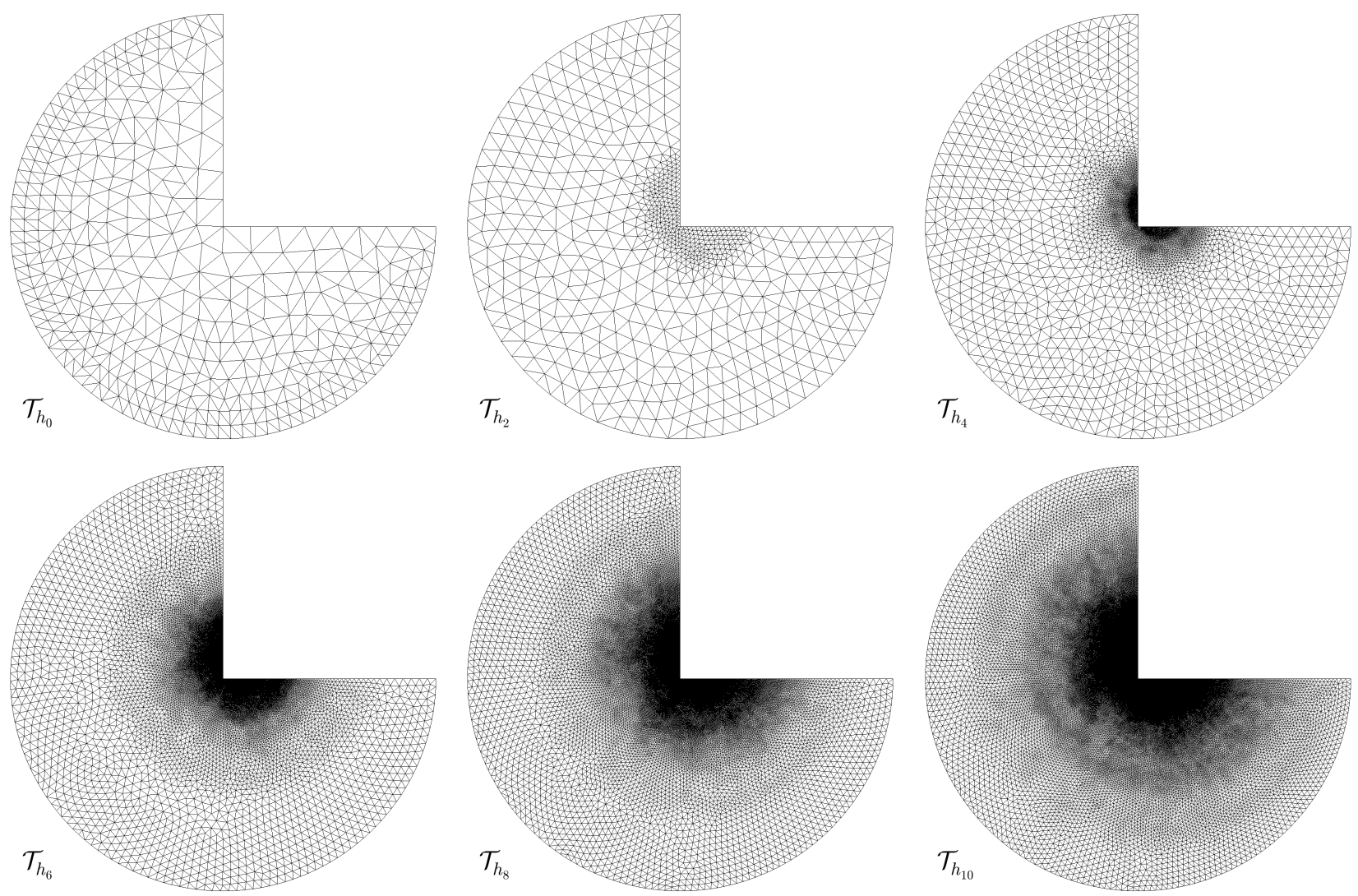

Figure 6.3: Example 2: Initial coarse mesh and adapted meshes after 2,4,6,8, and 10 iterations of Algorithm 1.

[13] Bernardi, C., Hecht, F. and Pironneau, O., Coupling Darcy and Stokes equations for porous media with cracks. ESAIM: Mathematical Modelling and Numerical Analysis, 39(1) (2005) 7-35.

[14] Braess, D. And Verfürth, R., A posteriori error estimators for the Raviart-Thomas element. SIAM Journal on Numerical Analysis, 33(6) (1996) 2431-2444.

[15] Brezzi, F. And Fortin, M., Mixed and Hybrid Finite Element Methods. Springer Verlag, 1991.

[16] Carstensen, C., An a posteriori error estimate for a first-kind integral equation. Mathematics of Computation, 66(217) (1997) 139-155.

[17] Carstensen, C., A-posteriori error estimate for the mixed finite element method. Mathematics of Computation, 66(218) (1997) 465-476.

[18] Chang, J. and Nakshatrala, K.B., Modification to Darcy model for high pressure and high velocity applications and associated mixed finite element formulations. arXiv:1306.5216[cs.NA]

[19] Ciarlet, P.G., The Finite Element Method for Elliptic Problems. North-Holland, Amsterdam, New York, Oxford, 1978.

[20] Clément, P., Approximation by finite element functions using local regularisation. RAIRO Modélisation Mathématique et Analyse Numérique, 9 (1975) 77-84. 

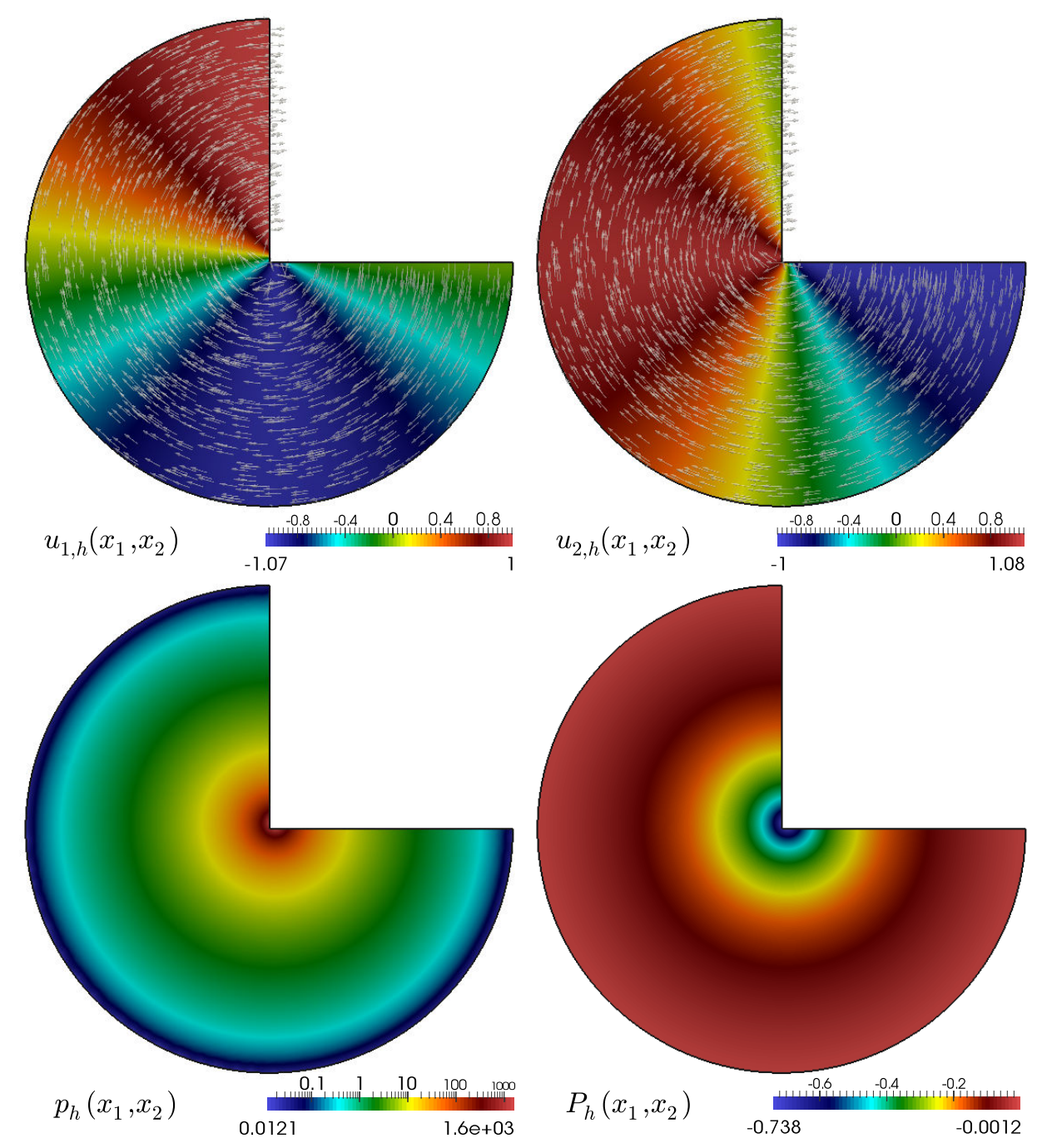

Figure 6.4: Example 2: Approximate velocity components (top), computed pressure distribution (bottom left) and postprocessed pressure (bottom right) obtained on an adapted mesh of 313041 vertices and 626080 elements.

[21] DaAdaA, M., Discrétisation Spectrale et par Éléments Spectraux des Équations de Darcy. Ph.D. Thesis, Université Pierre et Marie Curie, Paris (2009).

[22] Darcy, H., Les fontaines publiques de la ville de Dijon. Dalmont, Paris (1856).

[23] Discacciati, M., Miglio, E. and Quarteroni, A., Mathematical and numerical models for coupling surface and groundwater flows. Applied Numerical Mathematics, 43(1) (2002) 57-74.

[24] Domínguez, C., Gatica, G.N. And Meddahi, S., A posteriori error analysis of a fullymixed finite element method for a two-dimensional fluid-solid interaction problem. Preprint 201402, Centro de Investigación en Ingeniería Matemática ( $\left.\mathrm{CI}^{2} \mathrm{MA}\right)$, Universidad de Concepción, Concepción, Chile, (2014).

[25] Ervin, V.J., Jenkins, E.W. And Sun, S., Coupling nonlinear Stokes and Darcy flow using mortar finite elements. Applied Numerical Mathematics, 61(11) (2011) 1198-1222. 

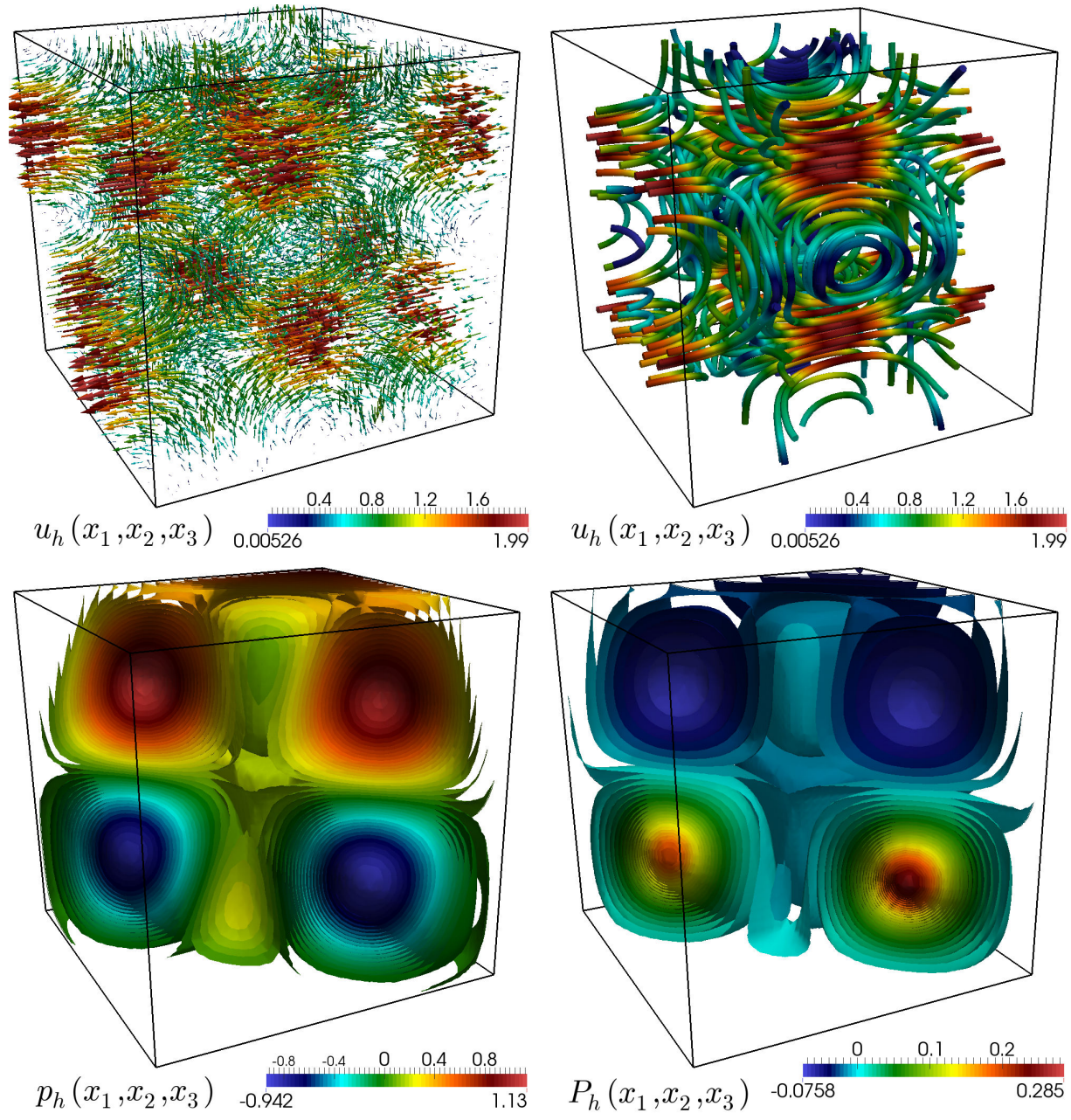

Figure 6.5: Example 2: Approximate velocity vectors and streamlines (top), computed pressure distribution (bottom left) and postprocessed pressure (bottom right) obtained on a uniform mesh of 286906 vertices and 573412 tetrahedral elements.

[26] Galvis, J. And SARkis, M., Non-matching mortar discretization analysis for the coupling Stokes-Darcy equations. Electronic Transactions on Numerical Analysis, 26 (2007) 350-384.

[27] Gatica, G.N., A Simple Introduction to the Mixed Finite Element Method. Theory and Applications. Springer Briefs in Mathematics, Springer, Cham Heidelberg New York Dordrecht London, 2014.

[28] Gatica, G.N., Gatica, L.F. and Márquez, A., Analysis of a pseudostress-based mixed finite element method for the Brinkman model of porous media flow. Numerische Mathematik, DOI:10.1007/s00211-013-0577-x.

[29] Gatica, G.N., Hsiao, G.C. And Meddahi, S., A residual-based a posteriori error estimator for a two-dimensional fluid-solid interaction problem. Numerische Mathematik, 114(1) (2009) 63-106. 
[30] Gatica, G.N. and Maischak, M., A posteriori error estimates for the mixed finite element method with Lagrange multipliers. Numerical Methods for Partial Differential Equations, 21(3) (2005) 421-450.

[31] Gatica, G.N., Meddahi, S. and Oyarzúa, R., A conforming mixed finite-element method for the coupling of fluid flow with porous media flow. IMA Journal of Numerical Analysis, 29(1) (2009) 86-108.

[32] Gatica, G.N., Oyarzúa, R. And Sayas, F.-J., Analysis of fully-mixed finite element methods for the Stokes-Darcy coupled problem. Mathematics of Computation, 80(276) (2011) 1911-1948.

[33] Girault V., Murat F., And Salgado A., Finite element discretization of Darcy's equations with pressure dependent porosity, ESAIM: Mathematical Modelling and Numerical Analysis, 44(6) (2010) 1155-1191.

[34] Girault, V. And Raviart, P.-A., Finite Element Methods for Navier-Stokes Equations. Theory and Algorithms. Springer Series in Computational Mathematics, vol. 5, Springer-Verlag, 1986.

[35] Girault, V. And Wheeler, M.F., Numerical discretization of a Darcy-Forchheimer model. Numerische Mathematik, 110(2) (2008) 161-198.

[36] Karakashian, O.A., On a Galerkin-Lagrange multiplier method for the stationary Navier Stokes equations. SIAM Journal on Numerical Analysis, 19(5) (1982) 909-923.

[37] Karper, T., Mardal, K.-A. And Winther, R., Unified finite element discretizations of coupled Darcy-Stokes flow. Numerical Methods for Partial Differential Equations, 25(2) (2009) 311-326.

[38] Lions, J.-L. And Magenes, E., Problèmes aux Limites non Homogènes et Applications I. Dunod, Paris, 1968.

[39] Márquez, A., Meddahi, S. And Sayas, F.-J., Strong coupling of finite element methods for the Stokes-Darcy problem. arXiv:1203.4717v1[math.NA]

[40] Mc Lean, W., Strongly Elliptic Systems and Boundary Integral Equations. Cambridge University Press, 2000.

[41] Nakshatrala, K.B. and Rajagopal K.R., A numerical study of fluids with pressure dependent viscosity flowing through a rigid porous medium. International Journal for Numerical Methods in Fluids, 67(3) (2011) 342-368.

[42] Nakshatrala, K.B. And Turner, D.Z., A mixed formulation for a modification to Darcy equation based on Picard linearization and numerical solutions to large-scale realistic problems. International Journal for Computational Methods in Engineering Science and Mechanics, 14(6) (2013) 524-541.

[43] NečAs, J., Les Méthodes Directes en Théorie des Équations Elliptiques. Mason, Paris, 1967.

[44] Pan, H. And Rui, H., Mixed element method for two-dimensional Darcy-Forchheimer model. Journal of Scientific Computing, 52(3) (2012) 563-587.

[45] PARK, E.-J., Mixed finite element methods for generalized Forchheimer flow in porous media. Numerical Methods for Partial Differential Equations, 21(2) (2005) 213-228. 
[46] Prössdorf, S. And Silbermann, B., Numerical Analysis for Integral and Related Operators Equations. Birkhäuser, Basel (1991).

[47] Rajagopal K.R., On a hierarchy of approximate models for flows of incompressible fluids through porous solids, Mathematical Models and Methods in the Applied Sciences, 17(2) (2007) 215-252.

[48] Riviere, B. And Yotov, I., Locally conservative coupling of Stokes and Darcy flows. SIAM Journal on Numerical Analysis, 42(5) (2005) 1959-1977.

[49] Roberts, J.E. And Thomas, J.M., Mixed and Hybrid Methods. In: Handbook of Numerical Analysis, P.G. Ciarlet and J.L. Lions (Eds.), vol. II, Finite Element Methods (Part 1), 1991, North-Holland, Amsterdam.

[50] Srinivasan S., Bonito, A. and Rajagopal, K.R., Flow of a fluid through a porous solid due to high pressure gradients. Journal of Porous Media, 16(3) (2013) 193-203.

[51] Srinivasan, S. and Rajagopal, K.R., A thermodynamic basis for the derivation of the Darcy, Forchheimer and Brinkman models for flows through porous media and their generalizations. International Journal of Nonlinear Mechanics, 58 (2014) 162-166.

[52] Verfürth, R., A posteriori error estimators for the Stokes problem. Numerische Mathematik, 55(3), (1989) 309-325.

[53] Verfürth, R., A posteriori error estimation and adaptive mesh-refinement techniques. Journal of Computational and Applied Mathematics, 50(1-3) (1994) 67-83.

[54] Verfürth, R., A Review of A-Posteriori Error Estimation and Adaptive Mesh-Refinement Techniques. John Wiley and Teubner Series. Advances in Numerical Mathematics 1996.

[55] Xie, X., Xu, J. And Xue, G., Uniformly stable finite element methods for Darcy-StokesBrinkman models. Journal of Computational Mathematics, 26(3) (2008) 437-455. 